\title{
Existence and Nonlinear Stability of Steady-States to Outflow Problem for the Full Two-Phase Flow
}

\author{
Hai-Liang Li ${ }^{a, b}$, Shuang Zhao ${ }^{a, b *}$, Han-Wen Zuo ${ }^{a, b}$ \\ ${ }^{a}$ School of Mathematical Sciences, Capital Normal University, Beijing 100048, P.R. China. \\ ${ }^{b}$ Academy for Multidisciplinary Studies, Capital Normal University, Beijing 100048, P.R. China.
}

\begin{abstract}
The outflow problem for the viscous full two-phase flow model in a half line is investigated in the present paper. The existence, uniqueness and nonlinear stability of the steady-state are shown respectively corresponding to the supersonic, sonic or subsonic state at far field. This is different from the outflow problem for the isentropic Navier-Stokes equations, where there is no steady-state for the subsonic state. Furthermore, we obtain either exponential time decay rates for the supersonic state or algebraic time decay rates for supersonic and sonic states in weighted Sobolev spaces.
\end{abstract}

Key words. Full two-phase flow, outflow problem, stationary solution, nonlinear stability.

\section{Introduction}

Two-phase flow models play important roles in applied scientific areas, for instance, nuclear, engines, chemical engineering, medicine, oil-gas, fluidization, waste water treatment, biomedical, liquid crystals, lubrication $[1,6,12,21,24]$, etc. In this paper, we consider the full two-phase flow model which can be formally obtained from a Vlasov-Fokker-Planck equation coupled with the compressible Navier-Stokes equations through the Chapman-Enskog expansion [19].

We consider the initial-boundary value problem (IBVP) for the full two-phase flow model as follows:

$$
\left\{\begin{array}{l}
\rho_{t}+(\rho u)_{x}=0 \\
(\rho u)_{t}+\left[\rho u^{2}+p_{1}(\rho)\right]_{x}=\mu u_{x x}+n(v-u), \\
n_{t}+(n v)_{x}=0 \\
(n v)_{t}+\left[n v^{2}+p_{2}(n)\right]_{x}=\left(n v_{x}\right)_{x}-n(v-u), \quad x>0, t>0,
\end{array}\right.
$$

\footnotetext{
*Corresponding author.

E-mail address: hailiang.li.math@gmail.com (H.-L. Li), shuangzhaomath@163.com (S. Zhao), zhw1880118@163.com (H.-W. Zuo).
} 
where $\rho>0$ and $n>0$ stand for the densities, $u$ and $v$ are the velocities of two fluids respectively, the constant $\mu>0$ is the viscosity coefficient, and the pressure-density functions take forms

$$
p_{1}(\rho)=A_{1} \rho^{\gamma}, \quad p_{2}(n)=A_{2} n^{\alpha}
$$

with $A_{1}>0, A_{2}>0, \gamma \geq 1$ and $\alpha \geq 1$. The initial data are given by

$$
\begin{gathered}
(\rho, u, n, v)(0, x)=\left(\rho_{0}, u_{0}, n_{0}, v_{0}\right)(x), \quad \inf _{x \in \mathbb{R}_{+}} \rho_{0}(x)>0, \quad \inf _{x \in \mathbb{R}_{+}} n_{0}(x)>0, \\
\lim _{x \rightarrow+\infty}\left(\rho_{0}, u_{0}, n_{0}, v_{0}\right)(x)=\left(\rho_{+}, u_{+}, n_{+}, u_{+}\right), \quad \rho_{+}>0, \quad n_{+}>0,
\end{gathered}
$$

and the outflow boundary condition is imposed by

$$
(u, v)(t, 0)=\left(u_{-}, u_{-}\right), \quad u_{-}<0,
$$

where $\rho_{+}>0, n_{+}>0, u_{+}$and $u_{-}<0$ are constants.

The condition $u_{-}<0$ means that the fluids flow out the region $\mathbb{R}_{+}$through the boundary $x=0$ with the velocity $u_{-}$, and therefore the problem (1.1)-(1.5) is called the outflow problem [22]. On the other hand, the similar problem with the case $u_{-}>0$ is called the inflow problem [22], and the densities on the boundary $(\rho, n)(t, 0)=\left(\rho_{-}, n_{-}\right)$are also imposed for the well-posedness of the inflow problem.

It is an interesting issue to study the outflow/inflow problem. There are many important progress made recently about the existence and nonlinear stability of steady-states and basic waves to the outflow/inflow problem for one-phase flow, such as compressible Navier-Stokes equations [3, 5, 8-11, 1518, 23, 26-34]. For instance, for the inflow problem of isentropic Navier-Stokes equations, MatsumuraNishihara [23] showed the existence and stability of steady-states for both subsonic and sonic cases together with the stability of the superposition of steady-states and rarefaction waves under small perturbation, while Fan-Liu-Wang-Zhao [5] investigated that steady-states and rarefaction waves were nonlinear stable under large perturbation, and Huang-Matsumura-Shi [9] obtained the stability of superposition of steady-states and shock waves under small perturbation. For the inflow problem of full compressible Navier-Stokes equations, Nakamura-Nishibata [26] got the existence and stability of steady-states also for both subsonic and sonic cases, and Qin-Wang [31,32] and Hong-Wang [8] showed the combination of steady-states and rarefaction waves. For the outflow problem of isentropic Navier-Stokes equations, the existence and nonlinear stability of steady-states for both supersonic and sonic cases under small perturbation were proved in [16,27-29], the convergence rates toward steady-states for supersonic and sonic cases were investigated in $[28,29]$, the stability of the superposition of steady-states and rarefaction waves were got in $[11,17]$. For the outflow problem of full compressible Navier-Stokes equations, KawashimaNakamura-Nishibata [15] established the existence and nonlinear stability of steady-states under small perturbation for three cases: supersonic, sonic and subsonic flow while there is no steady-state to the 
outflow problem of isentropic Navier-Stokes equations for the subsonic case, in addition they also gained the convergence rates toward the stationary solutions for supersonic and sonic cases [15], and Qin [30], Wan-Wang-Zhao [33] and Wan-Wang-Zou [34] obtained the stability of steady-states, rarefaction waves and their combination under large perturbation.

It is of interest and challenges to investigate the outflow/inflow problem for two-phase flow models due to the coupled motions of two phases. Although it is rather complicated, some important progress has been made about the existence and nonlinear stability of steady-states and basic waves to the outflow/inflow problem for two-phase flow models [4,7,20,35,37,38]. For instance, Yin-Zhu [37] obtained the existence of steady-states similar to isentropic Navier-Stokes equations [16], and the nonlinear stability and convergence rates of steady-states for the supersonic case to the outflow problem of the drift-flux model. For the outflow problem of the two-fluid Navier-Stokes-Poisson system, the existence of stedystates which is similar to that of isentropic Navier-Stokes equations [16], and the nonlinear stability of rarefactions waves and steady-states together with the superposition of steady-states and rarefaction waves were proved in $[4,35]$. We established existence and nonlinear stability of steady-states to inflow problem of the model (1.1) for supersonic, sonic and subsonic cases in [20], which is a different phenomena compared with the inflow problem for isentropic Navier-Stokes equations [23] and full compressible Navier-Stokes equations [26], where there is no steady-state for the supersonic case.

However, there is no result about the existence and nonlinear stability of steady-states for the outflow problem (1.1)-(1.5). The main purpose of this paper is to prove the existence and nonlinear stability of steady-states for the supersonic, sonic and subsonic case, and obtain either exponential time decay rates for the supersonic flow or algebraic time decay rates for both supersonic and sonic flows. Contrary to the isentropic Navier-Stokes equations, the steady-state to the IBVP (3.3)-(1.5) exists for the subsonic case.

The steady-state $(\widetilde{\rho}, \widetilde{u}, \widetilde{n}, \widetilde{v})(x)$ to the outflow problem (1.1)-(1.5) satisfies the following system

$$
\left\{\begin{array}{l}
(\widetilde{\rho u})_{x}=0, \\
{\left[\widetilde{\rho} \widetilde{u}^{2}+p_{1}(\widetilde{\rho})\right]_{x}=\left(\mu \widetilde{u}_{x}\right)_{x}+\widetilde{n}(\widetilde{v}-\widetilde{u}),} \\
(\widetilde{n} \widetilde{v})_{x}=0, \\
{\left[\widetilde{n} \widetilde{v}^{2}+p_{2}(\widetilde{n})\right]_{x}=\left(\widetilde{n} \widetilde{v}_{x}\right)_{x}-\widetilde{n}(\widetilde{v}-\widetilde{u}), \quad x>0,}
\end{array}\right.
$$

with the boundary conditions and spatial far field conditions

$$
(\widetilde{u}, \widetilde{v})(0)=\left(u_{-}, u_{-}\right), \quad \lim _{x \rightarrow \infty}(\widetilde{\rho}, \widetilde{u}, \widetilde{n}, \widetilde{v})(x)=\left(\rho_{+}, u_{+}, n_{+}, u_{+}\right), \quad \inf _{x \in \mathbb{R}_{+}} \widetilde{\rho}(x)>0, \quad \inf _{x \in \mathbb{R}_{+}} \widetilde{n}(x)>0 .
$$

Integrating $(1.6)_{1}$ and $(1.6)_{3}$ over $(x,+\infty)$, we have

$$
\widetilde{u}=\frac{\rho_{+}}{\widetilde{\rho}} u_{+}, \quad \widetilde{v}=\frac{n_{+}}{\widetilde{n}} u_{+}
$$


which implies that the following relationship

$$
u_{+}=\frac{\widetilde{n}(0)}{n_{+}} u_{-}=\frac{\widetilde{\rho}(0)}{\rho_{+}} u_{-}<0
$$

is the necessary property of the steady-state to the boundary value problem (BVP) (1.6)-(1.7).

Define the Mach number $M_{+}$and the sound speed $c_{+}$as below

$$
M_{+}:=\frac{\left|u_{+}\right|}{c_{+}}, \quad c_{+}:=\left(\frac{A_{1} \gamma \rho_{+}^{\gamma}+A_{2} \alpha n_{+}^{\alpha}}{\rho_{+}+n_{+}}\right)^{\frac{1}{2}} .
$$

Then, we have the following results about the existence and uniqueness of the steady-state.

Theorem 1.1. Let $\delta:=\left|u_{-}-u_{+}\right|>0$ and $u_{+}<0$ hold. Then there exists a set $\Omega_{-} \subset \mathbb{R}_{-}$such that if $u_{-} \in \Omega_{-}$and $\delta$ sufficiently small, there exists a unique strong solution $(\widetilde{\rho}, \widetilde{u}, \widetilde{n}, \widetilde{v})(x)$ to the BVP (1.6)-(1.7) which satisfies either for the supersonic or subsonic case $M_{+} \neq 1$ that

$$
\left|\partial_{x}^{k}\left(\widetilde{\rho}-\rho_{+}, \widetilde{u}-u_{+}, \widetilde{n}-n_{+}, \widetilde{v}-u_{+}\right)\right| \leq C_{1} \delta e^{-c_{0} x}, \quad k=0,1,2,3,
$$

or for the sonic case $M_{+}=1$ that

$$
\left|\partial_{x}^{k}\left(\widetilde{\rho}-\rho_{+}, \widetilde{u}-u_{+}, \widetilde{n}-n_{+}, \widetilde{v}-u_{+}\right)\right| \leq C_{2} \frac{\delta^{k+1}}{(1+\delta x)^{k+1}}, \quad k=0,1,2,3,
$$

and

$$
\left(\widetilde{u}_{x}, \widetilde{v}_{x}\right)=\left(a \sigma^{2}(x), a \sigma^{2}(x)\right)+O\left(|\sigma(x)|^{3}\right),
$$

where $\sigma(x)$ is a smooth function satisfying $\sigma_{x}=-a \sigma^{2}+O\left(|\sigma|^{3}\right)$ and

$$
c_{1} \frac{\delta}{1+\delta x} \leq \sigma(x) \leq C_{3} \frac{\delta}{1+\delta x}, \quad\left|\partial_{x}^{k} \sigma(x)\right| \leq C_{3} \frac{\delta^{k+1}}{(1+\delta x)^{k+1}}, \quad k=0,1,2,3,
$$

and $C_{i}>0, i=1,2,3, c_{0}>0, c_{1}>0$, and $a>0$ are positive constants.

Remark 1.1. Due to the drag force term, the existence of steady-states to the IBVP (1.1)-(1.5) is obtained even for the subsonic case $M_{+}<1$, which is different from that of the isentropic Navier-Stokes equations [16]. Moreover, the existence of steady-states to the IBVP (1.1)-(1.5) is similar to that of the full compressible Navier-Stokes equations [15].

Then, we have the nonlinear stability of the steady-state to the IBVP (1.1)-(1.5) for supersonic, sonic and subsonic cases.

Theorem 1.2. Let the same conditions in Theorem 1.1 hold and assume that it holds

$$
\left|p_{1}^{\prime}\left(\rho_{+}\right)-p_{2}^{\prime}\left(n_{+}\right)\right| \leq \sqrt{2}\left|u_{+}\right| \min \left\{\left(1+\frac{\rho_{+}}{n_{+}}\right)\left[(\gamma-1) p_{1}^{\prime}\left(\rho_{+}\right)\right]^{\frac{1}{2}},\left(1+\frac{n_{+}}{\rho_{+}}\right)\left[(\alpha-1) p_{2}^{\prime}\left(n_{+}\right)\right]^{\frac{1}{2}}\right\}
$$

for the sonic case $M_{+}=1$. Then, there exists a small positive constant $\varepsilon_{0}>0$ such that if

$$
\left\|\left(\rho_{0}-\widetilde{\rho}, u_{0}-\widetilde{u}, n_{0}-\widetilde{n}, v_{0}-\widetilde{v}\right)\right\|_{H^{1}}+\delta \leq \varepsilon_{0},
$$


the IBVP (1.1)-(1.5) has a unique global solution $(\rho, u, n, v)(t, x)$ satisfying

$$
\left\{\begin{array}{l}
(\rho-\widetilde{\rho}, u-\widetilde{u}, n-\widetilde{n}, v-\widetilde{v}) \in C\left([0,+\infty) ; H^{1}\right) \\
(\rho-\widetilde{\rho}, n-\widetilde{n})_{x} \in L^{2}\left([0,+\infty) ; L^{2}\right) \\
(u-\widetilde{u}, v-\widetilde{v})_{x} \in L^{2}\left([0,+\infty) ; H^{1}\right)
\end{array}\right.
$$

and

$$
\lim _{t \rightarrow+\infty} \sup _{x \in \mathbb{R}_{+}}|(\rho-\widetilde{\rho}, u-\widetilde{u}, n-\tilde{n}, v-\widetilde{v})(t, x)|=0 .
$$

In addition, we have the time convergence rates of the global solution to the IBVP (1.1)-(1.5) for both supersonic and sonic cases.

Theorem 1.3. Assume that the same conditions in Theorem 1.1 hold. Then, the following results hold.

(i) For $M_{+}>1$ and $\lambda>0$, if the initial data satisfy

$$
(1+x)^{\frac{\lambda}{2}}\left(\rho_{0}-\widetilde{\rho}, u_{0}-\widetilde{u}, n_{0}-\widetilde{u}, n-\widetilde{n}\right) \in L^{2}\left(\mathbb{R}_{+}\right)
$$

and

$$
\left\|\left(\rho_{0}-\widetilde{\rho}, u_{0}-\widetilde{u}, n_{0}-\widetilde{n}, v_{0}-\widetilde{v}\right)\right\|_{H^{1}}+\delta \leq \varepsilon_{0}
$$

for a small positive constant $\varepsilon_{0}>0$, then the solution $(\rho, u, n, v)(t, x)$ to the IBVP (1.1)-(1.5) satisfies

$$
\|(\rho-\widetilde{\rho}, u-\widetilde{u}, n-\widetilde{n}, v-\widetilde{v})(t)\|_{L^{\infty}} \leq C_{4} \delta_{0}(1+t)^{-\frac{\lambda}{2}}
$$

where $C_{4}>0$ and $\delta_{0}:=\left\|\left(\rho_{0}-\widetilde{\rho}, u_{0}-\widetilde{u}, n_{0}-\widetilde{n}, v_{0}-\widetilde{v}\right)\right\|_{H^{1}}+\left\|(1+x)^{\frac{\lambda}{2}}\left(\rho_{0}-\widetilde{\rho}, u_{0}-\widetilde{u}, n_{0}-\tilde{n}, v_{0}-\widetilde{v}\right)\right\|_{L^{2}}$ are constants independent of time.

(ii) For $M_{+}=1,1 \leq \lambda<\lambda^{*}, \lambda^{*}:=2+\sqrt{8+\frac{1}{1+b^{2}}}$ and $b:=\frac{\rho_{+}\left(u_{+}^{2}-p_{1}^{\prime}\left(\rho_{+}\right)\right)}{\left|u_{+}\right| \sqrt{\left(\mu+n_{+}\right) n_{+}}}$, if for arbitrary $\nu \in(0, \lambda]$, there exists a small positive constant $\varepsilon_{0}>0$ such that

$$
\left\|\sigma^{-\frac{\lambda}{2}}\left(\rho_{0}-\widetilde{\rho}, u_{0}-\widetilde{u}, n_{0}-\widetilde{n}, v_{0}-\widetilde{v}\right)\right\|_{H^{1}}+\delta^{\frac{1}{2}} \leq \varepsilon_{0}
$$

then the IBVP (1.1)-(1.5) has a unique global solution $(\rho, u, n, v)(t, x)$ satisfying

$$
\left\{\begin{array}{l}
\sigma^{-\frac{\nu}{2}}(\rho-\widetilde{\rho}, u-\widetilde{u}, n-\widetilde{n}, v-\widetilde{v}) \in C\left([0,+\infty): H^{1}\right), \\
\sigma^{-\frac{\nu}{2}}(\rho-\widetilde{\rho}, n-\widetilde{n})_{x} \in L^{2}\left([0,+\infty) ; L^{2}\right) \\
\sigma^{-\frac{\nu}{2}}(u-\widetilde{u}, v-\widetilde{v})_{x} \in L^{2}\left([0,+\infty) ; H^{1}\right)
\end{array}\right.
$$

and

$$
\left\|\sigma^{-\frac{\nu}{2}}(\rho-\widetilde{\rho}, u-\widetilde{u}, n-\widetilde{n}, v-\widetilde{v})(t)\right\|_{H^{1}} \leq C_{5} \delta_{1}(1+t)^{-\frac{\lambda-\nu}{4}}
$$

where $\sigma(x)$ is defined by (1.14) satisfying (2.28), $C_{5}>0$ is a positive constant independent of time, and $\delta_{1}:=\left\|\sigma^{-\frac{\lambda}{2}}\left(\rho_{0}-\widetilde{\rho}, u_{0}-\widetilde{u}, n_{0}-\widetilde{n}, v_{0}-\widetilde{v}\right)\right\|_{H^{1}}$ is a constant. 
Remark 1.2. For $M_{+}>1$, the exponential time convergence rates of the global solution to the IBVP (1.1)-(1.5) can be established. Indeed, assume that $M_{+}>1, u_{+}<0$ and a certain positive constant $\lambda>0$ hold. For a certain positive constant $\kappa \in(0, \lambda]$, there exists a small positive constant $\varepsilon_{0}>0$ such that if $e^{\frac{\lambda}{2} x}\left(n_{0}-\widetilde{n}, \rho_{0}-\widetilde{\rho}, u_{0}-\widetilde{u}, n_{0}-\widetilde{n}, v_{0}-\widetilde{v}\right) \in L^{2}\left(\mathbb{R}_{+}\right)$and

$$
\left.\|\left(\rho_{0}-\widetilde{\rho}, u_{0}-\widetilde{u}, n_{0}-\widetilde{n}, v_{0}-\widetilde{v}\right)\right) \|_{H^{1}}+\delta \leq \varepsilon_{0},
$$

then the solution $(\rho, u, n, v)(t, x)$ to the IBVP (1.1)-(1.5) satisfies

$$
\|(\rho-\widetilde{\rho}, u-\widetilde{u}, n-\widetilde{n}, v-\widetilde{v})(t)\|_{H^{1}} \leq C_{6} \delta_{2} e^{-\frac{\kappa_{1}}{2} t},
$$

where $C_{6}>0$ and $\kappa_{1} \ll \kappa$ are positive constants independent of time, and $\delta_{2}:=\|\left(\rho_{0}-\widetilde{\rho}, u_{0}-\widetilde{u}, n_{0}-\right.$ $\left.\widetilde{n}, v_{0}-\widetilde{v}\right)\left\|_{H^{1}}+\right\| e^{\frac{\lambda}{2} x}\left(\rho_{0}-\widetilde{\rho}, u_{0}-\widetilde{u}, n_{0}-\widetilde{n}, v_{0}-\widetilde{v}\right) \|_{L^{2}}$ is a constant.

The proof of (1.23) can be obtained by similar arguments as for (1.19). The details are omitted.

Remark 1.3. In Theorem 1.3, we remove the restriction (1.15), and obtain the nonlinear stability of steady-states and time decay rates of the solution to the IBVP (1.1)-(1.5) for $M_{+}=1$ with the weighted energy method. Moreover, if $p_{1}^{\prime}\left(\rho_{+}\right)=p_{2}^{\prime}\left(n_{+}\right)$, time decay rates of the solution to the IBVP (1.1)-(1.5) for $M_{+}=1$ are the same as that of isentropic Navier-Stokes equations [28].

We explain main strategies to prove Theorems 1.1-1.3. The system (1.1)-(1.5) can be viewed as two compressible isentropic Navier-Stokes equations coupled with each other through the drag force relaxation mechanisms. Different from the isentropic Navier-Stokes equations [16], we can not reformulate two momentum equations $(1.1)_{2}$ and $(1.1)_{4}$ into conservation forms due to the influence of drag force, which implies that the steady-state satisfies the system (1.6)-(1.7) consisting of a first-order and a second-order ordinary differential equations instead of only a first-order ordinary differential equation in [16], and it is not straightforward to apply the center manifold theory [2]. To overcome the difficulty, we introduce a new variable $\widetilde{w}:=\widetilde{u}_{x}$, get the estimate $\left|\widetilde{u}_{x}(0)\right| \leq C\left|u_{-}-u_{+}\right|$in Lemma 2.1, and then rewrite the system (1.6) into the $3 \times 3$ system (2.9) of autonomous ordinary differential equations. Since the condition $p_{1}^{\prime}\left(\rho_{+}\right)=p_{2}^{\prime}\left(n_{+}\right)$is not necessary, we need subtle analysis to obtain the sign of $\operatorname{Re} \lambda_{i}, i=1,2,3$, where $\lambda_{i}, i=1,2,3$ are three eigenvalues of the linearized $3 \times 3$ system of (2.9). It should be noticed that the linearized system of (2.9) has at least one eigenvalue with negative real part due to the effect of drag force, so that we obtain the existence of steady-states for the supersonic, sonic and subsonic case in Theorem 1.1, which is different from the outflow problem of the isentropic Navier-Stokes system [16], where there is no steady-state for the subsonic case.

We establish the uniform estimates of the perturbation $(\phi, \psi, \bar{\phi}, \bar{\psi}):=(\rho-\widetilde{\rho}, u-\widetilde{u}, n-\widetilde{n}, v-\widetilde{v})$ to prove the nonlinear stability of steady-states for the supersonic $M_{+}>1$, sonic case $M_{+}=1$ and subsonic case $M_{+}<1$. For $M_{+}=1$, it is easy to check that $\left(\widetilde{\rho} \widetilde{u}^{2}+p_{1}(\widetilde{\rho})\right)_{x}$ and $\left(\widetilde{n} \widetilde{v}^{2}+p_{2}(\widetilde{n})\right)_{x}$ decay slower than 
$\widetilde{u}_{x x}$ for $p_{1}^{\prime}\left(\rho_{+}\right) \neq p_{2}^{\prime}\left(n_{+}\right)$owing to $(1.6)_{2},(1.6)_{4}$ and (1.12), which implies the term

$$
\int R_{2} d x:=\int \phi \psi \frac{\left(\widetilde{\rho} \widetilde{u}^{2}+p_{1}(\widetilde{\rho})\right)_{x}}{\widetilde{\rho}}+\bar{\phi} \bar{\psi} \frac{\left(\widetilde{n} \widetilde{v}^{2}+p_{2}(\widetilde{n})\right)_{x}}{\widetilde{n}} d x
$$

can not be controlled directly as in [16]. With the help of $\widetilde{u}_{x} \geq 0$ and $\widetilde{v}_{x} \geq 0$, we turn to deal with the terms

$$
\int R_{2} d x+\int\left(\rho \psi^{2}+p_{1}(\rho)-p_{1}(\widetilde{\rho})-p_{1}^{\prime}(\widetilde{\rho}) \phi\right) \widetilde{u}_{x} d x+\left(n \bar{\psi}^{2}+p_{2}(n)-p_{2}(\widetilde{n})-p_{2}^{\prime}(\widetilde{n})\right) \widetilde{v}_{x} d x,
$$

the leading terms of which can be rewritten as two positive semidefinite 2 variable quadratic forms (3.21) under the condition (1.15).

By the weighted energy method, we get the exponential or algebraic time decay rates for the supersonic case $M_{+}>1$ if the initial perturbation belongs to the exponential or algebraic weighted Sobolev space, and obtain algebraic time decay rates for the sonic case $M_{+}=1$. To get the basic weighted energy estimates, we use (1.11) and the dissipation on the relaxation friction term $\bar{\psi}-\psi$, and decompose $\psi$ as $\psi=\bar{\psi}+(\psi-\bar{\psi})$, as motivated by Li-Wang-Wang [19]. Due to the algebraic decay (1.12) of steady-states, convergence rates of steady-states for the sonic case $M_{+}=1$ is worse than that of the supersonic case $M_{+}>1$. It is necessary to use the delicate algebraic decay (1.12)-(1.14) and the dissipation on the drag force $\bar{\psi}-\psi$, decompose $\psi$ as $\psi=\bar{\psi}+(\psi-\bar{\psi})$, and obtain more delicate estimates to get the convergence rates for $M_{+}=1$. It should be noticed that we make full use of the dissipation on drag force term and the viscous terms, and take a linear coordinate transformation

$$
\left(\begin{array}{c}
\phi \\
\bar{\phi} \\
\bar{\psi}
\end{array}\right)=\boldsymbol{P}\left(\begin{array}{l}
\hat{\rho} \\
\hat{n} \\
\hat{v}
\end{array}\right), \quad \text { with a invertible matrix } \boldsymbol{P},
$$

to gain the 3 variable quadratic form $\hat{\lambda}_{1} \hat{\rho}^{2}+\hat{\lambda}_{2} \hat{n}^{2}$ with $\hat{\lambda}_{1}, \hat{\lambda}_{2}>0$, which plays an important role in basic weighted energy estimates together with some crucial cancellations. In fact, we obtain the algebraic time decay rates of the solution to IBVP (1.1)-(1.5) for $M_{+}=1$ for the initial perturbation satisfying $\sigma^{-\frac{\lambda}{2}}\left(\rho_{0}-\widetilde{\rho}, u_{0}-\widetilde{u}, n_{0}-\widetilde{n}, v_{0}-\widetilde{v}\right) \in L^{2}\left(\mathbb{R}_{+}\right)$, with $\lambda<\lambda^{*}, \lambda^{*}:=2+\sqrt{8+\frac{1}{1+b^{2}}}, b=\frac{\rho_{+}\left(u_{+}^{2}-p_{1}^{\prime}\left(\rho_{+}\right)\right)}{\left|u_{+}\right| \sqrt{\left(\mu+n_{+}\right) n_{+}}}$ and the function $\sigma \geq 0$ satisfying (1.14). This is an interesting phenomena describing the influences of two fluids on each other somehow and it should be emphasized that $\lambda^{*}=5$ is the same as that of the isentropic Navier-Stokes equations [28] for $u_{+}^{2}=p_{1}^{\prime}\left(\rho_{+}\right)=p_{2}^{\prime}\left(n_{+}\right)$.

Notation. We denote by $\|\cdot\|_{L^{p}}$ the norm of the usual Lebesgue space $L^{p}=L^{p}\left(\mathbb{R}_{+}\right), 1 \leq p \leq \infty$. And if $p=2$, we write $\|\cdot\|_{L^{p}\left(\mathbb{R}_{+}\right)}=\|\cdot\| \cdot H^{s}\left(\mathbb{R}_{+}\right)$stands for the standard $s$-th Sobolev space over $\mathbb{R}_{+}$equipped with its norm

$$
\|f\|_{H^{s}\left(\mathbb{R}_{+}\right)}=\|f\|_{s}:=\left(\sum_{i=0}^{s}\left\|\partial^{i} f\right\|^{2}\right)^{\frac{1}{2}} .
$$


$C\left([0, T] ; H^{1}\left(\mathbb{R}_{+}\right)\right)$represents the space of continuous functions on the interval $[0, T]$ with values in $H^{s}\left(\mathbb{R}_{+}\right) . \quad L^{2}([0, T] ; \mathcal{B})$ denotes the space of $L^{2}$ functions on the interval $[0, T]$ with values in Banach space $\mathcal{B}$. For a scalar function $W(x)>0$, the weighted $L^{2}\left(\mathbb{R}_{+}\right)$and $H^{1}\left(\mathbb{R}_{+}\right)$spaces are defined as follows:

$$
\begin{aligned}
L_{W}^{2}\left(\mathbb{R}_{+}\right) & :=\left\{f \in L^{2}\left(\mathbb{R}_{+}\right) \mid\|f\|_{L_{W}^{2}}:=\left(\int_{\mathbb{R}_{+}} W(x) f^{2} d x\right)^{\frac{1}{2}}<+\infty\right\}, \\
H_{W}^{1}\left(\mathbb{R}_{+}\right): & =\left\{f \in H^{1}\left(\mathbb{R}_{+}\right) \mid\|f\|_{H_{W}^{1}}:=\left(\sum_{i=0}^{1}\left\|\partial^{i} f\right\|_{L_{W}^{2}}^{2}\right)^{\frac{1}{2}}<+\infty\right\} .
\end{aligned}
$$

For a scalar function $W_{a, \nu}:=(1+x)^{\nu}$ with $\nu \geq 0$, we denote $\|f\|_{a, \nu}:=\left\|(1+x)^{\frac{\nu}{2}} f\right\|$.

The rest of this paper will be organized as follows. We prove the existence and uniqueness of steadystates in Section 2, get the nonlinear stability of steady-states in Section 3 for supersonic, sonic and subsonic cases, and obtain convergence rates of steady-states for the supersonic flow in Subsection 4.1 and the sonic flow in Subsection 4.2.

\section{Existence of Steady-State}

We prove Theorem 1.1 on the existence and uniqueness of steady-states to the BVP (1.6)-(1.7) with $u_{+}<0$ and $\delta$ sufficiently small as follows. In order to apply the center manifold theory [2], it is necessary to get the bounds of $\widetilde{u}_{x}(0)$ or $\widetilde{v}_{x}(0)$.

Lemma 2.1. Assume that $u_{+}<0$ and $\delta=\left|u_{-}-u_{+}\right|$hold with $\delta$ sufficiently small. Then the steady-state $(\widetilde{\rho}, \widetilde{u}, \widetilde{n}, \widetilde{v})$ to the BVP (1.6)-(1.7) satisfies

$$
\left|\widetilde{u}_{x}(0)\right| \leq C\left|u_{-}-u_{+}\right|, \quad\left|\widetilde{v}_{x}(0)\right| \leq C\left|u_{-}-u_{+}\right|,
$$

where $C>0$ is a positive constant.

Proof. Due to $\widetilde{\rho}=\frac{\rho_{+} u_{+}}{\widetilde{u}}$ and $\widetilde{n}=\frac{n_{+} u_{+}}{\widetilde{v}}$, we have

$$
\left\{\begin{array}{l}
\left(\rho_{+} u_{+} \widetilde{u}+A_{1} \rho_{+}^{\gamma} u_{+}^{\gamma} \widetilde{u}^{-\gamma}\right)_{x}=\left(\mu \widetilde{u}_{x}\right)_{x}+\frac{n_{+} u_{+}}{\widetilde{v}}(\widetilde{v}-\widetilde{u}), \\
\left(n_{+} u_{+} \widetilde{v}+A_{2} n_{+}^{\alpha} u_{+}^{\alpha} \widetilde{v}^{-\alpha}\right)_{x}=\left(n_{+} u_{+} \frac{\widetilde{v}_{x}}{\widetilde{v}}\right)_{x}-\frac{n_{+} u_{+}}{\widetilde{v}}(\widetilde{v}-\widetilde{u}) .
\end{array}\right.
$$

Adding $(2.2)_{1}$ to $(2.2)_{2}$ and integrating the resulted equation over $(0,+\infty)$ lead to

$$
\mu \widetilde{u}_{x}(0)+\frac{n_{+} u_{+}}{u_{-}} \widetilde{v}_{x}(0)=\frac{1}{u_{+}}\left[\left(\rho_{+}+n_{+}\right) u_{+}^{2}-\left(A_{1} \gamma \rho_{+}^{\gamma}+A_{2} \alpha n_{+}^{\alpha}\right)\right]\left(u_{-}-u_{+}\right)+O\left(\left|u_{-}-u_{+}\right|^{2}\right) .
$$

With the help of $(2.3)$, we multiply $(2.2)_{1}$ by $\widetilde{u},(2.2)_{2}$ by $\widetilde{v}$ respectively, then integrate the summation of the resulted equations over $(0,+\infty)$ to gain

$$
\begin{aligned}
& \int_{0}^{+\infty}\left(\mu \widetilde{u}_{x}^{2}+n_{+} u_{+} \frac{\widetilde{v}_{x}^{2}}{\widetilde{v}}\right) d x+\int_{0}^{+\infty} \frac{n_{+} u_{+}}{\widetilde{v}}(\widetilde{v}-\widetilde{u})^{2} d x \\
= & -u_{-}\left[\mu \widetilde{u}_{x}(0)+n_{+} u_{+} \frac{\widetilde{v}_{x}(0)}{u_{-}}\right]+\left[\left(\rho_{+}+n_{+}\right) u_{+}^{2}-\left(A_{1} \gamma \rho_{+}^{\gamma}+A_{2} \alpha n_{+}^{\alpha}\right)\right]\left(u_{-}-u_{+}\right)+O\left(\left|u_{-}-u_{+}\right|^{2}\right) \\
= & O\left(\left|u_{-}-u_{+}\right|^{2}\right) .
\end{aligned}
$$


Multiplying $(2.2)_{2}$ by $\frac{\widetilde{v}_{x}}{\widetilde{v}}$ and then integrating the resulted equation over $(0, \infty)$ yield

$$
-n_{+} u_{+} \frac{\widetilde{v}_{x}^{2}(0)}{2 u_{-}^{2}}+\int_{0}^{+\infty} A_{2} \alpha n_{+}^{\alpha} \frac{u_{+}^{\alpha}}{\widetilde{v}^{\alpha+2}} \widetilde{v}_{x}^{2} d x=\int_{0}^{+\infty} \frac{n_{+} u_{+}}{\widetilde{v}}(\widetilde{v}-\widetilde{u}) \frac{\widetilde{v}_{x}}{\widetilde{v}} d x+\int_{0}^{+\infty} n_{+} u_{+} \frac{\widetilde{v}_{x}^{2}}{\widetilde{v}} d x
$$

We estimate terms in the right hand side of (2.5). With $\inf _{x \in \mathbb{R}_{+}} \widetilde{n}>0, \alpha \geq 1$ and (2.4), we have

$$
\begin{aligned}
& \int_{0}^{+\infty} \frac{n_{+} u_{+}}{\widetilde{v}}(\widetilde{v}-\widetilde{u}) \frac{\widetilde{v}_{x}}{\widetilde{v}} d x+\int_{0}^{+\infty} n_{+} u_{+} \frac{\widetilde{v}_{x}^{2}}{\widetilde{v}} d x \\
\leq & C \int_{0}^{+\infty} \frac{n_{+} u_{+}}{\widetilde{v}}(\widetilde{v}-\widetilde{u})^{2} d x+\frac{1}{4} \int_{0}^{+\infty} A_{2} \alpha n_{+}^{\alpha} \frac{u_{+}^{\alpha}}{\widetilde{v}^{\alpha+2}} \widetilde{v}_{x}^{2} d x \\
\leq & C\left|u_{-}-u_{+}\right|^{2}+\frac{1}{4} \int_{0}^{+\infty} A_{2} \alpha n_{+}^{\alpha} \frac{u_{+}^{\alpha}}{\widetilde{v}^{\alpha+2}} \widetilde{v}_{x}^{2} d x .
\end{aligned}
$$

Combining (2.5) and (2.6), we get

$$
\left|\widetilde{v}_{x}(0)\right| \leq C\left|u_{-}-u_{+}\right|, \quad\left|\widetilde{u}_{x}(0)\right| \leq C\left|u_{-}-u_{+}\right| .
$$

Then, we can prove Theorem 1.1 with the above lemma. For $(1.6)_{2}$ and $(1.6)_{4}$, using $\widetilde{\rho}=\frac{\rho_{+} u_{+}}{\widetilde{u}}$, $\widetilde{n}=\frac{n_{+} u_{+}}{\widetilde{v}}$ and integrating the summation of $(1.6)_{2}$ and $(1.6)_{4}$ over $(x,+\infty)$, we have

$$
\left\{\begin{array}{l}
\widetilde{v}_{x}=\frac{\widetilde{v}}{n_{+} u_{+}}\left[\rho_{+} u_{+}\left(\widetilde{u}-u_{+}\right)+A_{1} \rho_{+}^{\gamma}\left(\frac{u_{+}^{\gamma}}{\widetilde{u}^{\gamma}}-1\right)+n_{+} u_{+}\left(\widetilde{v}-u_{+}\right)+A_{2} n_{+}^{\alpha}\left(\frac{u_{+}^{\alpha}}{\widetilde{v}^{\alpha}}-1\right)-\mu \widetilde{u}_{x}\right], \\
\widetilde{u}_{x x}=\frac{1}{\mu}\left[\left(\rho_{+} u_{+}-A_{1} \rho_{+}^{\gamma} u_{+}^{\gamma} \widetilde{u}^{-\gamma-1}\right) \widetilde{u}_{x}-n_{+} u_{+}\left(1-\frac{\widetilde{u}}{\widetilde{v}}\right)\right] .
\end{array}\right.
$$

Define $\widetilde{w}:=\widetilde{u}_{x}$ and $\bar{U}:=(\bar{u}, \bar{w}, \bar{v})^{\mathrm{T}}:=\left(\widetilde{u}-u_{+}, \widetilde{w}, \widetilde{v}-u_{+}\right)^{\mathrm{T}}$. The system (2.8) can be reformulated into the autonomous system as follows

$$
\left\{\begin{array}{l}
\bar{U}_{x}=J_{+} \bar{U}+\left(0, \bar{g}_{2}(\bar{U}), \bar{g}_{3}(\bar{U})\right)^{\mathrm{T}}, \\
\bar{U}_{-}:=(\bar{u}, \bar{w}, \bar{v})^{\mathrm{T}}(0)=\left(u_{-}-u_{+}, \widetilde{u}_{x}(0), u_{-}-u_{+}\right)^{\mathrm{T}}, \lim _{x \rightarrow \infty} \bar{U}^{\mathrm{T}}=(0,0,0),
\end{array}\right.
$$

where

$$
\begin{gathered}
\boldsymbol{J}_{+}=\left(\begin{array}{ccc}
0 & 1 & 0 \\
\frac{n_{+}}{\mu} & \frac{\rho_{+} u_{+}^{2}-A_{1} \gamma \rho_{+}^{\gamma}}{\mu u_{+}} & -\frac{n_{+}}{\mu} \\
\frac{\rho_{+} u_{+}^{2}-A_{1} \gamma \rho_{+}^{\gamma}}{n_{+} u_{+}} & -\frac{\mu}{n_{+}} & \frac{n_{+} u_{+}^{2}-A_{2} \alpha n_{+}^{\alpha}}{n_{+} u_{+}}
\end{array}\right), \\
\bar{g}_{2}(\bar{U})=\frac{1}{2}\left(2 \frac{n_{+}}{\mu} \frac{1}{u_{+}} \bar{v}^{2}-2 \frac{n_{+}}{\mu} \frac{1}{u_{+}} \bar{u} \bar{v}+2 \frac{A_{1} \gamma(\gamma+1) \rho_{+}^{\gamma}}{\mu u_{+}^{2}} \bar{w} \bar{u}\right)+O\left(|\bar{U}|^{3}\right), \\
\bar{g}_{3}(\bar{U})= \\
=\frac{1}{2}\left[\frac{A_{1} \gamma(\gamma+1) \rho_{+}^{\gamma}}{n_{+} u_{+}^{2}} \bar{u}^{2}+2 \frac{\rho_{+} u_{+}^{2}-A_{1} \gamma \rho_{+}^{\gamma}}{n_{+} u_{+}^{2}} \bar{v} \bar{u}+\left(2 \frac{n_{+} u_{+}^{2}-A_{2} \alpha n_{+}^{\alpha}}{n_{+} u_{+}^{2}}\right.\right. \\
\left.\left.+\frac{A_{2} \alpha(\alpha+1) n_{+}^{\alpha}}{n_{+} u_{+}^{2}}\right) \bar{v}^{2}-2 \frac{\mu}{n_{+} u_{+}} \bar{w} \bar{v}\right]+O\left(|\bar{U}|^{3}\right) .
\end{gathered}
$$


Three eigenvalues $\lambda_{1}, \lambda_{2}, \lambda_{3}$ of matrix $\boldsymbol{J}_{+}$satisfy

$$
\left\{\begin{array}{l}
\lambda_{1} \lambda_{2} \lambda_{3}=-\frac{\left(\rho_{+}+n_{+}\right) u_{+}^{2}-\left(A_{1} \gamma \rho_{+}^{\gamma}+A_{2} \alpha n_{+}^{\alpha}\right)}{\mu u_{+}}, \\
\lambda_{1}+\lambda_{2}+\lambda_{3}=\frac{\rho_{+} u_{+}^{2}-A_{1} \gamma \rho_{+}^{\gamma}}{\mu u_{+}}+\frac{n_{+} u_{+}^{2}-A_{2} \alpha n_{+}^{\alpha}}{n_{+} u_{+}}, \\
\lambda_{1} \lambda_{2}+\lambda_{1} \lambda_{3}+\lambda_{2} \lambda_{3}=\rho_{+} \frac{\left(u_{+}^{2}-A_{1} \gamma \rho_{+}^{\gamma-1}\right)\left(u_{+}^{2}-A_{2} \alpha n_{+}^{\alpha-1}\right)}{\mu u_{+}^{2}}-1-\frac{n_{+}}{\mu} .
\end{array}\right.
$$

If $M_{+}>1$, it is easy to obtain $\lambda_{1} \lambda_{2} \lambda_{3}>0$ and $u_{+}^{2}>\min \left\{A_{1} \gamma \rho_{+}^{\gamma-1}, A_{2} \alpha n_{+}^{\alpha-1}\right\}$. Without loss of generality, we assume $A_{1} \gamma \rho_{+}^{\gamma-1} \geq A_{2} \alpha n_{+}^{\alpha-1}$. Moreover, we have

$$
\lambda_{1}+\lambda_{2}+\lambda_{3}<0 \text { for } u_{+}^{2}>A_{1} \gamma \rho_{+}^{\gamma-1}, \quad \lambda_{1} \lambda_{2}+\lambda_{1} \lambda_{3}+\lambda_{2} \lambda_{3}<0 \text { for } A_{2} \alpha n_{+}^{\alpha-1} \leq u_{+}^{2} \leq A_{1} \gamma \rho_{+}^{\gamma-1},
$$

which can imply $\operatorname{Re} \lambda_{1}<0, \operatorname{Re} \lambda_{2}<0$ and $\lambda_{3}>0$ for $M_{+}>1$. Using similar arguments, we have the following results:

$$
\left\{\begin{array}{l}
\text { if } M_{+}>1, \text { then } \operatorname{Re} \lambda_{1}<0, \operatorname{Re} \lambda_{2}<0, \lambda_{3}>0, \\
\text { if } M_{+}<1, \text { then } \operatorname{Re} \lambda_{1}>0, \operatorname{Re} \lambda_{2}>0, \lambda_{3}<0 \\
\text { if } M_{+}=1 \text {, then } \lambda_{1}>0, \lambda_{2}<0, \lambda_{3}=0 .
\end{array}\right.
$$

Then, applying the center manifold theory [2], it is not difficult to show the supersonic or subsonic case $M_{+} \neq 1$ in Theorem 1.1 if $\delta$ is small. Finally, we prove the sonic case $M_{+}=1$ in Theorem 1.1 which implies $\lambda_{1}>0, \lambda_{2}<0, \lambda_{3}=0$. The eigenvectors $r_{1}, r_{2}, r_{3}$ of $\lambda_{1}, \lambda_{2}, \lambda_{3}$ are obtained respectively as follows

$$
r_{1}=\left(\begin{array}{c}
1 \\
\lambda_{1} \\
-\frac{\mu}{n_{+}}\left(\lambda_{1}^{2}-\frac{\rho_{+} u_{+}^{2}-A_{1} \gamma \rho_{+}^{\gamma}}{\mu u_{+}} \lambda_{1}\right)+1
\end{array}\right), r_{2}=\left(\begin{array}{c}
1 \\
\lambda_{2} \\
-\frac{\mu}{n_{+}}\left(\lambda_{2}^{2}-\frac{\rho_{+} u_{+}^{2}-A_{1} \gamma \rho_{+}^{\gamma}}{\mu u_{+}} \lambda_{2}\right)+1
\end{array}\right), r_{3}=\left(\begin{array}{l}
1 \\
0 \\
1
\end{array}\right) .
$$

Define the matrix $\boldsymbol{P}_{\mathbf{1}}:=\left[r_{1}, r_{2}, r_{3}\right]$ and take a linear transformation $Z:=\left(z_{1}, z_{2}, z_{3}\right)^{\mathrm{T}}=\boldsymbol{P}_{\mathbf{1}}^{-1} \bar{U}$. With (2.11) and (2.12), the system (2.9) can be reformulated as follows

$$
\left\{\begin{array}{l}
\frac{d}{d x}\left(\begin{array}{c}
z_{1} \\
z_{2} \\
z_{3}
\end{array}\right)=\left(\begin{array}{ccc}
\lambda_{1} & * & 0 \\
0 & \lambda_{2} & 0 \\
0 & 0 & \lambda_{3}
\end{array}\right)\left(\begin{array}{l}
z_{1} \\
z_{2} \\
z_{3}
\end{array}\right)+\left(\begin{array}{l}
g_{1}\left(z_{1}, z_{2}, z_{3}\right) \\
g_{2}\left(z_{1}, z_{2}, z_{3}\right) \\
g_{3}\left(z_{1}, z_{2}, z_{3}\right)
\end{array}\right) \\
\left(z_{1}, z_{2}, z_{3}\right)(0)=\left(z_{1-}, z_{2-}, z_{3-}\right)=\left(P_{1}^{-1} \bar{U}_{-}\right)^{\mathrm{T}}, \quad \lim _{x \rightarrow \infty}\left(z_{1}, z_{2}, z_{3}\right)=(0,0,0),
\end{array}\right.
$$

where nonlinear functions $g_{i}(i=1,2,3)$ are denoted by

$$
\left(\begin{array}{l}
g_{1}\left(z_{1}, z_{2}, z_{3}\right) \\
g_{2}\left(z_{1}, z_{2}, z_{3}\right) \\
g_{3}\left(z_{1}, z_{2}, z_{3}\right)
\end{array}\right)=\boldsymbol{P}_{1}^{-1}\left(\begin{array}{c}
0 \\
\bar{g}_{2}(\bar{u}, \bar{w}, \bar{v}) \\
\bar{g}_{3}(\bar{u}, \bar{w}, \bar{v})
\end{array}\right)
$$


With the help of the manifold theory [2], there exist a local center manifold $W^{c}(0,0,0)$ and a local stable manifold $W_{3}^{s}(0,0,0)$

$$
\begin{aligned}
& W^{c}(0,0,0)=\left\{\left(z_{1}, z_{2}, z_{3}\right)\left|z_{1}=f_{1}^{c}\left(z_{3}\right), z_{2}=f_{2}^{c}\left(z_{3}\right),\right| z_{3} \mid \text { sufficient small }\right\}, \\
& W_{3}^{s}(0,0,0)=\left\{\left(z_{1}, z_{2}, z_{3}\right)\left|z_{1}=f_{1}^{s}\left(z_{2}\right), z_{3}=f_{2}^{s}\left(z_{2}\right),\right| z_{2} \mid \text { sufficient small }\right\},
\end{aligned}
$$

where $f_{i}^{c}, f_{i}^{s}, i=1,2$ are smooth functions and $f_{i}^{c}(0)=0, D f_{i}^{c}(0)=0, f_{i}^{s}(0)=0, D f_{i}^{s}(0)=0, i=1,2$. Using $\bar{U}=P Z,(2.21)-(2.12)$, and (2.18), we gain

$$
\bar{g}_{3}\left(z_{3}\right)=a z_{3}^{2}+O\left(\left|z_{1}\right|^{2}+\left|z_{2}\right|^{2}+\left|z_{3}\right|^{3}+\left|z_{1} z_{3}\right|+\left|z_{2} z_{3}\right|\right),
$$

where

$$
a=\frac{A_{1} \gamma(\gamma+1) \rho_{+}^{\gamma}+A_{2} \alpha(\alpha+1) n_{+}^{\alpha}}{2 u_{+}^{2}\left(1+b^{2}\right)\left(\mu+n_{+}\right)}, \quad b:=\frac{\rho_{+}\left(u_{+}^{2}-p_{1}^{\prime}\left(\rho_{+}\right)\right)}{\left|u_{+}\right| \sqrt{\left(\mu+n_{+}\right) n_{+}}} .
$$

Therefore, the system (2.17) can be reformulated as follows

$$
\left\{\begin{array}{l}
z_{1 x}=\lambda_{1} z_{1}+O\left(|Z|^{2}\right), \\
z_{2 x}=\lambda_{2} z_{2}+O\left(|Z|^{2}\right), \\
z_{3 x}=a z_{3}^{2}+O\left(\left|z_{1}\right|^{2}+\left|z_{2}\right|^{2}+\left|z_{3}\right|^{3}+\left|z_{1} z_{3}\right|+\left|z_{2} z_{3}\right|\right), \\
\left(z_{1}, z_{2}, z_{3}\right)(0):=\left(z_{1-}, z_{2-}, z_{3-}\right)=\left(\boldsymbol{P}_{1}^{-1} \bar{U}_{-}\right)^{\mathrm{T}}, \lim _{x \rightarrow \infty}\left(z_{1}, z_{2}, z_{3}\right)=(0,0,0) .
\end{array}\right.
$$

Let $\sigma_{1}(x)$ be a solution to $(2.23)_{1}$ restricted on the local center manifold satisfying the equation

$$
\sigma_{1 x}=a \sigma_{1}^{2}+O\left(\sigma_{1}^{3}\right), \quad \sigma_{1}(x) \rightarrow 0 \text { as } x \rightarrow+\infty .
$$

which implies that there exists the monotonically increasing solution $\sigma_{1}(x)<0$ to $(2.24)$ if $\sigma_{1}(0)<0$ holds and $\left|\sigma_{1}(0)\right|$ is sufficiently small. Therefore, if the initial data $\left(z_{1-}, z_{2-}, z_{3-}\right)$ belongs to the region $\mathcal{M} \subset \mathbb{R}^{3}$ associated to the local stable manifold and the local center manifold, then we have

$$
\left\{\begin{array}{l}
z_{i}=O\left(\sigma_{1}^{2}\right)+O\left(\delta e^{-c x}\right), i=1,2, \\
z_{3}=\sigma_{1}+O\left(\delta e^{-c x}\right),
\end{array}\right.
$$

with $z_{3-}<0$, the smallness of $\left|\left(z_{1-}, z_{2-}, z_{3-}\right)\right|$ and

$$
c \frac{\delta}{1+\delta x} \leq\left|\sigma_{1}\right| \leq C \frac{\delta}{1+\delta x}, \quad\left|\partial^{k} \sigma_{1}\right| \leq C \frac{\delta^{k+1}}{(1+\delta x)^{k+1}}, \quad C>0, \quad k=0,1,2,3 .
$$

Due to $\sigma_{1}(x) \leq 0$, we define

$$
\sigma(x):=-\sigma_{1},
$$

which satisfies

$$
\sigma_{x}=-a \sigma^{2}+O\left(|\sigma|^{3}\right), \quad \sigma \rightarrow 0 \text { as } x \rightarrow+\infty
$$


It is easy to get

$$
\left|\partial_{x}^{k}\left(\widetilde{\rho}-\rho_{+}, \widetilde{u}-u_{+}, \widetilde{n}-n_{+}, \widetilde{v}-u_{+}\right)\right| \leq C \frac{\delta^{k+1}}{(1+\delta x)^{k+1}}, \quad C>0, \quad k=0,1,2,3,
$$

and

$$
\left(\widetilde{u}-u_{+}, \widetilde{v}-u_{+}\right)=(-\sigma(x),-\sigma(x))+O\left(|\sigma(x)|^{2}\right), \quad\left(\widetilde{u}_{x}, \widetilde{v}_{x}\right)=\left(a \sigma^{2}(x), a \sigma^{2}(x)\right)+O\left(|\sigma|^{3}\right),
$$

with the help of $\bar{U}=\boldsymbol{P} Z$ and (2.16).

\section{Nonlinear stability of steady-states}

The function space $Y(0, T)$ for $T>0$ is denoted by

$$
\begin{aligned}
& Y(0, T):=\{(\phi, \psi, \bar{\phi}, \bar{\psi}) \mid(\phi, \psi, \bar{\phi}, \bar{\psi}) \in C\left([0, T] ; H^{1}\left(\mathbb{R}_{+}\right)\right), \\
&\left.\left(\phi_{x}, \bar{\phi}_{x}\right) \in L^{2}\left([0, T] ; L^{2}\left(\mathbb{R}_{+}\right)\right),\left(\psi_{x}, \bar{\psi}_{x}\right) \in L^{2}\left([0, T] ; H^{1}\left(\mathbb{R}_{+}\right)\right)\right\} .
\end{aligned}
$$

Let

$$
\phi=\rho-\widetilde{\rho}, \quad \psi=u-\widetilde{u}, \quad \bar{\phi}=n-\widetilde{n}, \quad \bar{\psi}=v-\widetilde{v} .
$$

Then the perturbation $(\phi, \psi, \bar{\phi}, \bar{\psi})$ satisfies the following system

$$
\left\{\begin{array}{l}
\phi_{t}+u \phi_{x}+\rho \psi_{x}=-\left(\psi \widetilde{\rho}_{x}+\phi \widetilde{u}_{x}\right) \\
\psi_{t}+u \psi_{x}+\frac{p_{1}^{\prime}(\rho)}{\rho} \phi_{x}-\frac{\mu \psi_{x x}}{\rho}-\frac{n(\bar{\psi}-\psi)}{\rho}=F_{1} \\
\bar{\phi}_{t}+v \bar{\phi}_{x}+n \bar{\psi}_{x}=-\left(\bar{\psi} \widetilde{n}_{x}+\bar{\phi}_{x}\right), \\
\bar{\psi}_{t}+v \bar{\psi}_{x}+\frac{p_{2}^{\prime}(n)}{n} \bar{\phi}_{x}-\frac{\left(n \bar{\psi}_{x}\right)_{x}}{n}+(\bar{\psi}-\psi)=F_{2},
\end{array}\right.
$$

where

$$
\begin{aligned}
& F_{1}=-\left[-\mu\left(\frac{1}{\rho}-\frac{1}{\widetilde{\rho}}\right) \widetilde{u}_{x x}+\psi \widetilde{u}_{x}+\left(\frac{p_{1}^{\prime}(\rho)}{\rho}-\frac{p_{1}^{\prime}(\widetilde{\rho})}{\widetilde{\rho}}\right) \widetilde{\rho}_{x}-\left(\frac{n}{\rho}-\frac{\widetilde{n}}{\widetilde{\rho}}\right)(\widetilde{v}-\widetilde{u})\right], \\
& F_{2}=-\left[-\left(\frac{1}{n}-\frac{1}{\widetilde{n}}\right)\left(\widetilde{n} \widetilde{v}_{x}\right)_{x}-\frac{\left(\bar{\phi} \widetilde{v}_{x}\right)_{x}}{n}+\bar{\psi} \widetilde{v}_{x}+\left(\frac{p_{2}^{\prime}(n)}{n}-\frac{p_{2}^{\prime}(\widetilde{n})}{\widetilde{n}}\right) \widetilde{n}_{x}\right] .
\end{aligned}
$$

The initial and boundary conditions to the system (3.3) satisfy

$$
\begin{gathered}
(\phi, \psi, \bar{\phi}, \bar{\psi})(0, x):=\left(\phi_{0}, \psi_{0}, \bar{\phi}_{0}, \bar{\psi}_{0}\right)=\left(\rho_{0}-\widetilde{\rho}, u_{0}-\widetilde{u}, n_{0}-\widetilde{n}, v_{0}-\widetilde{v}\right), \\
\lim _{x \rightarrow \infty}\left(\phi_{0}, \psi_{0}, \bar{\phi}_{0}, \bar{\psi}_{0}\right)=(0,0,0,0), \quad(\psi, \bar{\psi})(t, 0)=(0,0) .
\end{gathered}
$$

Proposition 3.1. Assume that the same assumptions in Theorem 1.2 hold. Let $(\phi, \psi, \bar{\phi}, \bar{\psi})$ be the solution to the problem (3.3)-(3.7) satisfying $(\phi, \psi, \bar{\phi}, \bar{\psi}) \in Y(0, T)$ for any time $T>0$. Then there exist positive constants $\varepsilon>0$ and $C>0$ independent of $T$ such that if

$$
\sup _{0 \leq t \leq T}\|(\phi, \psi, \bar{\phi}, \bar{\psi})(t)\|_{1}+\delta \leq \varepsilon
$$


is satisfied, then it holds for arbitrary $t \in[0, T]$ that

$$
\|(\phi, \psi, \bar{\phi}, \bar{\psi})\|_{1}^{2}+\int_{0}^{t}\left\|\left(\phi_{x}, \psi_{x}, \bar{\phi}_{x}, \bar{\psi}_{x}\right)\right\|^{2} d \tau+\int_{0}^{t}\left\|\left(\bar{\psi}-\psi, \psi_{x x}, \bar{\psi}_{x x}\right)\right\|^{2} d \tau \leq C\left\|\left(\phi_{0}, \psi_{0}, \bar{\phi}_{0}, \bar{\psi}_{0}\right)\right\|_{1}^{2} .
$$

With the help of (3.8), it is easy to verify the following Sobolev inequality

$$
\|(\phi, \psi, \bar{\phi}, \bar{\psi})(t)\|_{L^{\infty}} \leq\|(\phi, \psi, \bar{\phi}, \bar{\psi})(t)\|_{H^{1}} \leq \sqrt{2} \varepsilon
$$

Lemma 3.2 ( [16] ). For any function $\psi(t, \cdot) \in H^{1}\left(\mathbb{R}_{+}\right)$, it holds

$$
\begin{gathered}
\int_{0}^{\infty} \delta e^{-c_{0} x}|\psi|^{2} d x \leq C \delta\left(|\psi(t, 0)|^{2}+\left\|\psi_{x}(t)\right\|^{2}\right), \\
\int_{0}^{\infty} \frac{\delta^{j}}{(1+\delta x)^{j}}|\psi|^{2} d x \leq C \delta^{j-2}\left(|\psi(t, 0)|^{2}+\left\|\psi_{x}(t)\right\|^{2}\right), \quad j>2
\end{gathered}
$$

where $\delta>0, c_{0}>0$ and $C>0$ are positive constants.

With the Lemma 3.2, we can gain the basic $L^{2}$ energy estimates of $(\phi, \psi, \bar{\phi}, \bar{\psi})$.

Lemma 3.3. Under the same conditions in Proposition 3.1 , then the solution $(\phi, \psi, \bar{\phi}, \bar{\psi})$ to the problem (3.3)-(3.7) satisfies for $t \in[0, T]$ that

$$
\begin{aligned}
& \|(\phi, \psi, \bar{\phi}, \bar{\psi})\|^{2}+\int_{0}^{t}\left\|\left(\psi_{x}, \bar{\psi}_{x}, \bar{\psi}-\psi\right)\right\|^{2} d \tau+\int_{0}^{t}|\phi(t, 0)|^{2}+|\bar{\phi}(t, 0)|^{2} d \tau \\
\leq & C\left\|\left(\phi_{0}, \psi_{0}, \bar{\phi}_{0}, \bar{\psi}_{0}\right)\right\|^{2}+C(\delta+\varepsilon) \int_{0}^{t}\left\|\left(\phi_{x}, \bar{\phi}_{x}\right)\right\|^{2} d \tau .
\end{aligned}
$$

Proof. Define

$$
\begin{aligned}
\Phi_{1}(\rho, \widetilde{\rho})=\int_{\widetilde{\rho}}^{\rho} \frac{p_{1}(s)-p_{1}(\widetilde{\rho})}{s^{2}} d s, & \mathcal{E}_{1}=\rho\left(\frac{\psi^{2}}{2}+\Phi_{1}\right), \\
\Phi_{2}(n, \widetilde{n})=\int_{\widetilde{n}}^{n} \frac{p_{2}(s)-p_{2}(\widetilde{n})}{s^{2}} d s, & \mathcal{E}_{2}=n\left(\frac{\bar{\psi}^{2}}{2}+\Phi_{2}\right) .
\end{aligned}
$$

Then, by (1.1) and (1.6), the direct computations lead to

$$
\left(\mathcal{E}_{1}+\mathcal{E}_{2}\right)_{t}+\left(G_{1}+G_{2}\right)_{x}+n(\bar{\psi}-\psi)^{2}+\mu \psi_{x}^{2}+n \bar{\psi}_{x}^{2}+R_{1}+R_{2}=-R_{3},
$$

where

$$
\left\{\begin{array}{l}
G_{1}:=u \mathcal{E}_{1}+v \mathcal{E}_{2}+\left(p_{1}(\rho)-p_{1}(\widetilde{\rho})\right) \psi+\left(p_{2}(n)-p_{2}(\widetilde{n})\right) \bar{\psi} \\
G_{2}:=-\left(\mu \psi \psi_{x}+n \bar{\psi} \bar{\psi}_{x}+\bar{\phi} \bar{\psi} \widetilde{v}_{x}\right) \\
R_{1}:=\left[\rho \psi^{2}+p_{1}(\rho)-p_{1}(\widetilde{\rho})-p_{1}^{\prime}(\widetilde{\rho}) \phi\right] \widetilde{u}_{x}+\left[n \bar{\psi}^{2}+p_{2}(n)-p_{2}(\widetilde{n})-p_{2}^{\prime}(\widetilde{n}) \bar{\phi}\right] \widetilde{v}_{x} \\
R_{2}:=\phi \psi \frac{\tilde{\rho} \tilde{u} \widetilde{u}_{x}+\left(p_{1}(\widetilde{\rho})\right)_{x}}{\widetilde{\rho}}+\bar{\phi} \bar{\psi} \frac{\tilde{n} \widetilde{v} \widetilde{v}_{x}+\left(p_{2}(\widetilde{n})\right)_{x}}{\widetilde{n}} \\
R_{3}:=\bar{\phi}(\bar{\psi}-\psi)(\widetilde{v}-\widetilde{u})+\bar{\phi} \bar{\psi}_{x} \widetilde{v}_{x} .
\end{array}\right.
$$

Integrating (3.16) in $x$ over $\mathbb{R}_{+}$leads to

$$
\frac{d}{d t} \int \mathcal{E}_{1}+\mathcal{E}_{2} d x-G_{1}(t, 0)+\int n(\bar{\psi}-\psi)^{2}+\mu \psi_{x}^{2}+n \bar{\psi}_{x}^{2} d x+\int R_{1} d x+\int R_{2} d x=-\int R_{3} d x
$$


Under the condition (3.7), we get

$$
-G_{1}(t, 0)=-u_{-}\left[\Phi_{1}(\rho(t, 0), \widetilde{\rho}(0))+\Phi_{2}(n(t, 0), \widetilde{n}(0))\right] \geq c\left(\phi^{2}(t, 0)+\bar{\phi}^{2}(t, 0)\right) .
$$

For the supersonic or subsonic case $M_{+} \neq 1$, with the help of (1.11), (3.10) and (3.11), we have

$$
\int_{0}^{\infty}\left|R_{1}\right|+\left|R_{2}\right|+\left|R_{3}\right| d x \leq C \delta\left\|\left(\phi_{x}, \psi_{x}, \bar{\phi}_{x}, \bar{\psi}_{x}, \bar{\psi}-\psi\right)\right\|^{2}+C \delta\left(\phi^{2}(t, 0)+\bar{\phi}^{2}(t, 0)\right) .
$$

For the sonic case $M_{+}=1$ and the restriction $\left|p_{1}^{\prime}\left(\rho_{+}\right)-p_{2}^{\prime}\left(n_{+}\right)\right| \leq \sqrt{2}\left|u_{+}\right| \min \left\{\left(1+\frac{\rho_{+}}{n_{+}}\right)\left[(\gamma-1) p_{1}^{\prime}\left(\rho_{+}\right)\right]^{\frac{1}{2}},(1+\right.$ $\left.\left.\frac{n_{+}}{\rho_{+}}\right)\left[(\alpha-1) p_{2}^{\prime}\left(n_{+}\right)\right]^{\frac{1}{2}}\right\}$, using (1.12)-(1.14), (3.10), and (3.12), we get

$$
\begin{aligned}
& \int R_{1}+R_{2}+R_{3} d x \\
\geq & \int(\psi, \phi) \boldsymbol{M}_{\mathbf{1}}(\psi, \phi)^{\mathrm{T}} \widetilde{u}_{x}+(\bar{\psi}, \bar{\phi}) \boldsymbol{M}_{\mathbf{2}}(\bar{\psi}, \bar{\phi})^{\mathrm{T}} \widetilde{v}_{x} d x-C \int \frac{\delta^{3}}{(1+\delta x)^{3}}\left(\phi^{2}+\psi^{2}+\bar{\phi}^{2}+\bar{\psi}^{2}\right) d x \\
& -C \delta \int|\bar{\psi}-\psi|^{2}+\psi_{x}^{2} d x-C \delta^{\frac{1}{2}}\|(\phi, \psi, \bar{\phi}, \bar{\psi})\|\left[\phi^{2}(t, 0)+\bar{\phi}^{2}(t, 0)+\left\|\left(\phi_{x}, \psi_{x}, \bar{\phi}_{x}, \bar{\phi}_{x}\right)\right\|^{2}\right] \\
\geq & -C(\delta+\varepsilon)\left\|\left(\phi_{x}, \psi_{x}, \bar{\phi}_{x}, \bar{\psi}_{x}, \bar{\psi}-\psi\right)\right\|^{2}-C \delta\left(\phi^{2}(t, 0)+\bar{\phi}^{2}(t, 0)\right),
\end{aligned}
$$

where $\boldsymbol{M}_{\mathbf{1}}, \boldsymbol{M}_{\mathbf{2}}$ are positive definite or non-negative definite matrices defined by

$$
\boldsymbol{M}_{\mathbf{1}}=\left(\begin{array}{cc}
\rho_{+} & \frac{u_{+}^{2}-A_{1} \gamma \rho_{+}^{\gamma-1}}{2 u_{+}} \\
\frac{u_{+}^{2}-A_{1} \gamma \rho_{+}^{\gamma-1}}{2 u_{+}} & \frac{A_{1} \gamma(\gamma-1) \rho_{+}^{\gamma-2}}{2}
\end{array}\right), \quad \boldsymbol{M}_{\mathbf{2}}=\left(\begin{array}{cc}
n_{+} & \frac{u_{+}^{2}-A_{2} \alpha n_{+}^{\alpha-1}}{2 u_{+}} \\
\frac{u_{+}^{2}-A_{2} \alpha n_{+}^{\alpha-1}}{2 u_{+}} & \frac{A_{2} \alpha(\alpha-1) n_{+}^{\alpha-2}}{2}
\end{array}\right) .
$$

Finally, with the help of (3.18)-(3.21), we get (3.13). Hence, the proof of Lemma 3.3 is completed.

In order to complete the proof of Proposition 3.1, we need to establish the high order estimates of $(\phi, \psi, \bar{\phi}, \bar{\psi})$.

Lemma 3.4. Under the same conditions in Proposition 3.1, then the solution $(\phi, \psi, \bar{\phi}, \bar{\psi})$ to the problem (3.3)-(3.7) satisfies for $t \in[0, T]$ that

$$
\begin{aligned}
& \left\|\left(\phi_{x}, \bar{\phi}_{x}\right)\right\|^{2}+\int_{0}^{t}\left\|\left(\phi_{x}, \bar{\phi}_{x}\right)\right\|^{2} d \tau+\int_{0}^{t} \phi_{x}^{2}(t, 0)+\bar{\phi}_{x}^{2}(t, 0) d \tau \\
\leq & C\left\|\left(\phi_{0}, \psi_{0}, \phi_{0 x}, \bar{\phi}_{0}, \bar{\psi}_{0}, \bar{\phi}_{0 x}\right)\right\|^{2}+C(\varepsilon+\delta) \int_{0}^{t}\left\|\left(\psi_{x x}, \bar{\psi}_{x x}\right)\right\|^{2} d \tau .
\end{aligned}
$$

Proof. Differentiating $(3.3)_{1}$ in $x$, then multiplying the resulted equation by $\mu \phi_{x},(3.3)_{2}$ by $\widetilde{\rho}^{2} \phi_{x}$ respectively, we gain

$$
\begin{aligned}
& \left(\mu \frac{\phi_{x}^{2}}{2}\right)_{t}+\left(\mu u \frac{\phi_{x}^{2}}{2}\right)_{x}+\mu \widetilde{\rho} \phi_{x} \psi_{x x} \\
= & -\mu\left[\frac{3}{2} \psi_{x} \phi_{x}^{2}+\phi \phi_{x} \psi_{x x}+\left(\frac{1}{2} \phi_{x} \widetilde{u}_{x}+\psi_{x} \widetilde{\rho}_{x}\right) \phi_{x}+\left(\phi \widetilde{u}_{x}+\psi \widetilde{\rho}_{x}\right)_{x} \phi_{x}\right], \\
& \left(\widetilde{\rho}^{2} \phi_{x} \psi\right)_{t}-\left(\widetilde{\rho}^{2} \phi_{t} \psi\right)_{x}+\widetilde{\rho}^{2} \frac{p_{1}^{\prime}(\rho)}{\rho} \phi_{x}^{2}-\mu \widetilde{\rho} \phi_{x} \psi_{x x} \\
= & -\left(\widetilde{\rho}^{2} \phi_{t} \psi_{x}+\widetilde{\rho}^{2} u \phi_{x} \psi_{x}+2 \widetilde{\rho} \widetilde{\rho}_{x} \phi_{t} \psi\right)+\mu \widetilde{\rho}^{2}\left(\frac{1}{\rho}-\frac{1}{\widetilde{\rho}}\right) \phi_{x} \psi_{x x}+\widetilde{\rho}^{2} \frac{n}{\rho}(\bar{\psi}-\psi) \phi_{x}+F_{1} \widetilde{\rho}^{2} \phi_{x} .
\end{aligned}
$$


Similarly, differentiating $(3.3)_{3}$ in $x$, then multiplying the resulted equation by $\bar{\phi}_{x},(3.3)_{4}$ by $\widetilde{n} \bar{\phi}_{x}$ respectively lead to

$$
\begin{aligned}
& \left(\frac{\bar{\phi}_{x}^{2}}{2}\right)_{t}+\left(v \frac{\bar{\phi}_{x}^{2}}{2}\right)_{x}+\widetilde{n} \bar{\phi}_{x} \bar{\psi}_{x x}=-\left[\frac{3}{2} \bar{\psi}_{x} \bar{\phi}_{x}^{2}+\bar{\phi} \bar{\phi}_{x} \bar{\psi}_{x x}+\left(\frac{1}{2} \bar{\phi}_{x} \widetilde{v}_{x}+\bar{\psi}_{x} \widetilde{n}_{x}\right) \bar{\phi}_{x}-\left(\bar{\phi} \widetilde{v}_{x}+\bar{\psi} \widetilde{n}_{x}\right)_{x} \bar{\phi}_{x}\right], \\
& \left(\widetilde{n} \bar{\phi}_{x} \bar{\psi}\right)_{t}-\left(\widetilde{n} \bar{\phi}_{t} \bar{\psi}\right)_{x}+\widetilde{n} \frac{p_{2}^{\prime}(n)}{n} \bar{\phi}_{x}^{2}-\widetilde{n} \bar{\phi}_{x} \bar{\psi}_{x x} \\
= & -\left(\widetilde{n} \bar{\phi}_{t} \bar{\psi}_{x}+\widetilde{n} v \bar{\phi}_{x} \bar{\psi}_{x}+\widetilde{n}_{x} \bar{\phi}_{t} \bar{\psi}\right)+\left[\widetilde{n} \frac{\left(\bar{\phi} \bar{\psi}_{x}\right)_{x}}{n}+\widetilde{n}\left(\frac{1}{n}-\frac{1}{\widetilde{n}}\right)\left(\widetilde{n} \bar{\psi}_{x}\right)_{x}-\widetilde{n}(\bar{\psi}-\psi)\right] \bar{\phi}_{x} \\
& +\widetilde{n}_{x} \bar{\phi}_{x} \bar{\psi}_{x x}+F_{2} \widetilde{n} \bar{\phi}_{x} .
\end{aligned}
$$

Adding (3.24)-(3.27) together, integrating the resulted equation over $\mathbb{R}_{+}$, we have

$$
\begin{aligned}
& \frac{d}{d t} \int\left(\mu \frac{\phi_{x}^{2}}{2}+\frac{\bar{\phi}_{x}^{2}}{2}+\widetilde{\rho}^{2} \phi_{x} \psi+\widetilde{n} \bar{\phi}_{x} \bar{\psi}\right) d x+\int\left(\mu u \frac{\phi_{x}^{2}}{2}+v \frac{\bar{\phi}_{x}^{2}}{2}-\widetilde{\rho}^{2} \phi_{t} \psi-\widetilde{n} \bar{\phi}_{t} \bar{\psi}\right)_{x} d x \\
& +\int\left(\widetilde{\rho}^{2} \frac{p_{1}^{\prime}(\rho)}{\rho} \phi_{x}^{2}+\widetilde{n} \frac{p_{2}^{\prime}(n)}{n} \bar{\phi}_{x}^{2}\right) d x \\
= & \sum_{i=1}^{4} J_{i},
\end{aligned}
$$

where

$$
\begin{aligned}
J_{1}= & -\int\left[\widetilde{\rho}^{2}\left(\phi_{t}+u \phi_{x}\right) \psi_{x}+\widetilde{n}\left(\bar{\phi}_{t}+v \bar{\phi}_{x}\right) \bar{\psi}_{x}+2 \widetilde{\rho} \widetilde{\rho}_{x} \phi_{t} \psi+\widetilde{n}_{x} \bar{\phi}_{t} \bar{\psi}\right] d x, \\
J_{2}= & \int-\mu \phi \phi_{x} \psi_{x x}-\bar{\phi} \bar{\phi}_{x} \bar{\psi}_{x x}+\mu \widetilde{\rho}^{2}\left(\frac{1}{\rho}-\frac{1}{\widetilde{\rho}}\right) \phi_{x} \psi_{x x}+\widetilde{n}\left(\frac{1}{n}-\frac{1}{\widetilde{n}}\right)\left(\widetilde{n} \bar{\psi}_{x}\right)_{x} \bar{\phi}_{x}+\widetilde{\rho}^{2} \frac{n}{\rho}(\bar{\psi}-\psi) \phi_{x}-\widetilde{n}(\bar{\psi}-\psi) \bar{\phi}_{x} d x, \\
J_{3}= & -\int \frac{3}{2} \mu \psi_{x} \frac{\phi_{x}^{2}}{2}+\frac{3}{2} \bar{\psi}_{x} \bar{\phi}_{x}^{2} d x+\int \widetilde{n} \frac{\left(\bar{\phi} \bar{\psi}_{x}\right)_{x}}{n} \bar{\phi}_{x} d x \\
J_{4}= & -\int\left[\mu\left(\frac{1}{2} \phi_{x} \widetilde{u}_{x}+\psi_{x} \widetilde{\rho}_{x}\right) \phi_{x}+\mu\left(\phi \widetilde{u}_{x}+\psi \widetilde{\rho}_{x}\right)_{x} \phi_{x}-F_{1} \widetilde{\rho}^{2} \phi_{x}\right. \\
& \left.+\left(\frac{1}{2} \bar{\phi}_{x} \widetilde{v}_{x}+\bar{\psi}_{x} \widetilde{n}_{x}\right) \bar{\phi}_{x}-\left(\bar{\phi} \widetilde{v}_{x}+\bar{\psi} \widetilde{n}_{x}\right)_{x} \bar{\phi}_{x}-\widetilde{n}_{x} \bar{\phi}_{x} \bar{\psi}_{x x}-F_{2} \widetilde{n} \bar{\phi}_{x}\right] d x .
\end{aligned}
$$

First, we estimate terms in the left side of (3.28). Under the condition (3.7), the terms in the left side is estimated as follows

$$
\begin{gathered}
\int\left(\mu u \frac{\phi_{x}^{2}}{2}+v \frac{\bar{\phi}_{x}^{2}}{2}-\widetilde{\rho}^{2} \phi_{t} \psi-\widetilde{n} \bar{\phi}_{t} \bar{\psi}\right)_{x} d x=-u_{-} \frac{\mu \phi_{x}^{2}(0, t)+\bar{\phi}_{x}^{2}(0, t)}{2} \geq 0, \\
\int \widetilde{\rho}^{2} \frac{p_{1}^{\prime}(\rho)}{\rho} \phi_{x}^{2}+\widetilde{n} \frac{p_{2}^{\prime}(n)}{n} \bar{\phi}_{x}^{2} d x \geq \rho_{+} p_{1}^{\prime}\left(\rho_{+}\right)\left\|\phi_{x}\right\|^{2}+p_{2}^{\prime}\left(n_{+}\right)\left\|\bar{\phi}_{x}\right\|^{2}-C(\varepsilon+\delta)\left\|\left(\phi_{x}, \bar{\phi}_{x}\right)\right\|^{2} .
\end{gathered}
$$

We turn to estimate terms in the right hand side of (3.28). By (1.11)-(1.12), (3.3) $,(3.3)_{3},(3.11)-(3.12)$, Cauchy-Schwartz inequality and Young inequality with $0<\eta<1$, we obtain

$$
\begin{aligned}
\left|J_{1}\right| & \leq C\left\|\left(\psi_{x}, \bar{\psi}_{x}\right)\right\|^{2}+C \delta\left\|\left(\phi_{x}, \bar{\phi}_{x}\right)\right\|^{2}+C \delta\left(\phi^{2}(t, 0)+\bar{\phi}^{2}(t, 0)\right), \\
\left|J_{2}\right| & \leq C\|(\phi, \bar{\phi})\|_{L^{\infty}}\left\|\left(\phi_{x}, \bar{\phi}_{x}, \psi_{x x}, \bar{\psi}_{x x}\right)\right\|^{2}+C_{\eta}\|\bar{\psi}-\psi\|^{2}+\eta\left\|\left(\phi_{x}, \bar{\phi}_{x}\right)\right\|^{2}+C \delta\left\|\left(\bar{\phi}_{x}, \bar{\psi}_{x}\right)\right\|^{2} \\
& \leq C(\varepsilon+\delta+\eta)\left\|\left(\phi_{x}, \bar{\phi}_{x}\right)\right\|^{2}+C \varepsilon\left\|\left(\psi_{x x}, \bar{\psi}_{x x}\right)\right\|^{2}+C_{\eta}\|\bar{\psi}-\psi\|^{2}+C \delta\left\|\bar{\psi}_{x}\right\|^{2}, \\
\left|J_{3}\right| & \leq C\left\|\left(\psi_{x}, \bar{\psi}_{x}\right)\right\|_{L^{\infty}}\left\|\left(\phi_{x}, \bar{\phi}_{x}\right)\right\|^{2}+C\|\bar{\phi}\|_{L^{\infty}}\left\|\left(\bar{\phi}_{x}, \bar{\psi}_{x x}\right)\right\|^{2} \\
& \leq C \varepsilon\left\|\left(\phi_{x}, \psi_{x}, \bar{\phi}_{x}, \bar{\psi}_{x}\right)\right\|^{2}+C \varepsilon\left\|\left(\psi_{x x}, \bar{\psi}_{x x}\right)\right\|^{2},
\end{aligned}
$$




$$
\left|J_{4}\right| \leq C \delta\left\|\left(\phi_{x}, \psi_{x}, \bar{\phi}_{x}, \bar{\psi}_{x}\right)\right\|^{2}+C \delta\left\|\bar{\psi}_{x x}\right\|^{2}+C \delta\left(\phi^{2}(t, 0)+\bar{\phi}^{2}(t, 0)\right)
$$

Finally, the substitution of (3.29)-(3.34) into (3.28) for $\delta, \varepsilon$ and $\eta$ small enough leads to

$$
\begin{aligned}
& \frac{d}{d t} \int\left(\phi_{x}^{2}+\bar{\phi}_{x}^{2}+\widetilde{\rho}^{2} \phi_{x} \psi+\widetilde{n} \bar{\phi}_{x} \bar{\psi}\right) d x+\left\|\left(\phi_{x}, \bar{\phi}_{x}\right)\right\|^{2}+\phi_{x}^{2}(t, 0)+\bar{\phi}_{x}^{2}(t, 0) \\
\leq & C\left\|\left(\psi_{x}, \bar{\psi}_{x}, \bar{\psi}_{x}-\psi_{x}\right)\right\|^{2}+C(\delta+\varepsilon)\left\|\left(\psi_{x x}, \bar{\psi}_{x x}\right)\right\|^{2}+C \delta\left(\phi^{2}(t, 0)+\bar{\phi}^{2}(t, 0)\right) .
\end{aligned}
$$

Integrating (3.35) in $\tau$ over $[0, t]$, and using Lemma 3.3 and Young inequality, we have (3.23).

Lemma 3.5. Under the same conditions in Proposition 3.1, then the solution $(\phi, \psi, \bar{\phi}, \bar{\psi})$ to the problem (3.3)-(3.7) satisfies for $t \in[0, T]$ that

$$
\left\|\left(\psi_{x}, \bar{\psi}_{x}\right)\right\|^{2}+\int_{0}^{t}\left\|\left(\psi_{x x}, \bar{\psi}_{x x}\right)\right\|^{2} d \tau \leq C\left\|\left(\phi_{0}, \psi_{0}, \bar{\phi}_{0}, \bar{\psi}_{0}\right)\right\|_{1}^{2} .
$$

Proof. Multiplying $(3.3)_{2}$ by $-\psi_{x x},(3.3)_{4}$ by $-\bar{\psi}_{x x}$, respectively, then adding them together and integrating the resulted equation in $x$ over $\mathbb{R}_{+}$imply

$$
\frac{d}{d t} \int \frac{\psi_{x}^{2}}{2}+\frac{\bar{\psi}_{x}^{2}}{2} d x+\int \mu \frac{1}{\rho} \psi_{x x}^{2}+\bar{\psi}_{x x}^{2} d x=\sum_{i=1}^{3} K_{i}
$$

where

$$
\begin{aligned}
K_{1}= & \int\left[u \psi_{x} \psi_{x x}-\frac{n}{\rho}(\bar{\psi}-\psi) \psi_{x x}+\frac{p_{1}^{\prime}(\rho)}{\rho} \phi_{x} \psi_{x x}+v \bar{\psi}_{x} \bar{\psi}_{x x}+(\bar{\psi}-\psi) \bar{\psi}_{x x}+\frac{p_{2}^{\prime}(n)}{n} \bar{\phi}_{x} \bar{\psi}_{x x}\right] d x, \\
K_{2}= & \int\left[\widetilde{u}_{x} \psi \psi_{x x}-\mu \widetilde{u}_{x x}\left(\frac{1}{\rho}-\frac{1}{\widetilde{\rho}}\right) \psi_{x x}+\left(\frac{p_{1}^{\prime}(\rho)}{\rho}-\frac{p_{1}^{\prime}(\widetilde{\rho})}{\widetilde{\rho}}\right) \psi_{x x} \widetilde{\rho}_{x}-\left(\frac{n}{\rho}-\frac{\widetilde{n}}{\widetilde{\rho}}\right)(\widetilde{v}-\widetilde{u}) \psi_{x x}+\widetilde{v}_{x} \bar{\psi} \bar{\psi}_{x x}\right. \\
& \left.+\left(\widetilde{n} \widetilde{v}_{x}\right)_{x}\left(\frac{1}{n}-\frac{1}{\widetilde{n}}\right) \bar{\psi}_{x x}+\left(\frac{p_{2}^{\prime}(n)}{n}-\frac{p_{2}^{\prime}(\widetilde{n})}{\widetilde{n}}\right) \bar{\psi}_{x x} \widetilde{n}_{x}-\frac{\widetilde{n}_{x}}{\widetilde{n}} \bar{\psi}_{x} \bar{\psi}_{x x}-\frac{\left(\bar{\phi} \widetilde{v}_{x}\right)_{x}}{n} \bar{\psi}_{x x}\right] d x, \\
K_{3}= & -\int\left[\frac{\left(\bar{\phi} \bar{\psi}_{x}\right)_{x}}{n} \bar{\psi}_{x x}+\left(\frac{1}{n}-\frac{1}{\widetilde{n}}\right)\left(\widetilde{n} \bar{\psi}_{x}\right)_{x} \bar{\psi}_{x x}\right] d x .
\end{aligned}
$$

We estimate terms in the left side of (3.37). By the decomposition $\frac{1}{\rho}=\left(\frac{1}{\rho}-\frac{1}{\widetilde{\rho}}\right)+\left(\frac{1}{\tilde{\rho}}-\frac{1}{\rho_{+}}\right)+\frac{1}{\rho_{+}}$, the second term is estimated as follows:

$$
\begin{aligned}
\int \frac{\mu}{\rho} \psi_{x x}^{2}+\bar{\psi}_{x x}^{2} d x & \geq \frac{\mu}{\rho_{+}}\left\|\psi_{x x}\right\|^{2}+\left\|\bar{\psi}_{x x}\right\|^{2}-C\left(\|\phi\|_{L^{\infty}}+\delta\right)\left\|\psi_{x x}\right\|^{2} \\
& \geq \frac{\mu}{\rho_{+}}\left\|\psi_{x x}\right\|^{2}+\left\|\bar{\psi}_{x x}\right\|^{2}-C(\varepsilon+\delta)\left\|\psi_{x x}\right\|^{2} .
\end{aligned}
$$

We turn to estimate terms in the right side of (3.37). With the aid of (1.11), Sobolev inequality and Cauchy-Schwarz inequality, we have

$$
\begin{aligned}
& \left|K_{1}\right| \leq \frac{\mu}{16 \rho_{+}}\left\|\psi_{x x}\right\|^{2}+\frac{1}{16}\left\|\bar{\psi}_{x x}\right\|^{2}+C\left\|\left(\phi_{x}, \psi_{x}, \bar{\phi}_{x}, \bar{\psi}_{x}, \bar{\psi}-\psi\right)\right\|^{2}, \\
& \left|K_{2}\right| \leq C \delta\left\|\left(\phi_{x}, \psi_{x}, \bar{\phi}_{x}, \bar{\psi}_{x}\right)\right\|^{2}+C \delta\left\|\left(\psi_{x x}, \bar{\psi}_{x x}\right)\right\|^{2}+C \delta\left(\phi^{2}(t, 0)+\bar{\phi}^{2}(t, 0)\right), \\
& \left|K_{3}\right| \leq C\|\bar{\phi}\|_{L^{\infty}}\left\|\left(\bar{\psi}_{x}, \bar{\psi}_{x x}\right)\right\|^{2}+C\left\|\bar{\psi}_{x}\right\|_{L^{\infty}}\left\|\bar{\psi}_{x x}\right\|\left\|\bar{\phi}_{x}\right\| \leq C \varepsilon\left\|\left(\bar{\psi}_{x}, \bar{\psi}_{x x}\right)\right\|^{2} .
\end{aligned}
$$


Finally, taking $\delta$ and $\varepsilon$ small enough and substituting of (3.38)-(3.41) into (3.37), we obtain

$$
\frac{d}{d t} \int \psi_{x}^{2}+\bar{\psi}_{x}^{2} d x+\frac{\mu}{2 \rho_{+}}\left\|\psi_{x x}\right\|^{2}+\frac{1}{2}\left\|\bar{\psi}_{x x}\right\|^{2} \leq C\left\|\left(\phi_{x}, \psi_{x}, \bar{\phi}_{x}, \bar{\psi}_{x}, \bar{\psi}-\psi\right)\right\|^{2}+C \delta\left(\phi^{2}(t, 0)+\bar{\phi}^{2}(t, 0)\right) .
$$

Integrating (3.42) in $\tau$ over $[0, t]$, and using Lemmas 3.3-3.4 and the smallness of $\delta$ and $\varepsilon$, we obtain the desired estimate (3.36). Therefore, we complete the proof of Lemma 3.5.

With the help of Lemmas 3.3-3.5, we get (3.9) and complete the proof of Proposition 3.1.

\section{Time convergence rates}

\subsection{Convergence rate of supersonic steady-state}

Proposition 4.1. Assume that the same conditions in Theorem 1.3 for $M_{+}>1$ hold and let $(\phi, \psi, \bar{\phi}, \bar{\psi})$ be a solution to the $I B V P(3.3)-(3.7)$ satisfying $(\phi, \psi, \bar{\phi}, \bar{\psi}) \in C\left([0, T] ; H^{1}\right)$ and $(1+x)^{\frac{\nu}{2}}(\phi, \psi, \bar{\phi}, \bar{\psi}) \in$ $C\left([0, T] ; L^{2}\right)$ for any time $T>0$. Then for arbitrary $\nu \in[0, \lambda]$, there exist positive constants $\varepsilon>0$ and $C>0$ independent of $T$ such that if

$$
\sup _{0 \leq t \leq T}\|(\phi, \psi, \bar{\phi}, \bar{\psi})(t)\|_{1}+\delta \leq \varepsilon
$$

is satisfied, it holds for arbitrary $t \in[0, T]$ that

$$
\begin{aligned}
& (1+t)^{\lambda-\nu+\theta}\left(\|(\phi, \psi, \bar{\phi}, \bar{\psi})\|_{1}+\|(\phi, \psi, \bar{\phi}, \bar{\psi})\|_{a, \nu}^{2}\right)+\nu \int_{0}^{t}(1+\tau)^{\lambda-\nu+\theta}\|(\phi, \bar{\phi}, \psi, \bar{\psi})\|_{a, \nu-1}^{2} d \tau \\
& +\int_{0}^{t}(1+\tau)^{\lambda-\nu+\theta}\left\|\left(\psi_{x}, \bar{\psi}_{x}, \bar{\psi}-\psi\right)\right\|_{a, \nu}^{2} d \tau+\int_{0}^{t}(1+\tau)^{\lambda-\nu+\theta}\left\|\left(\phi_{x}, \psi_{x x}, \bar{\phi}_{x}, \bar{\psi}_{x x}\right)\right\|^{2} d \tau \\
\leq & C(1+t)^{\theta}\left(\left\|\left(\phi_{0}, \psi_{0}, \bar{\phi}_{0}, \bar{\psi}_{0}\right)\right\|_{1}^{2}+\left\|\left(\phi_{0}, \psi_{0}, \bar{\phi}_{0}, \bar{\psi}_{0}\right)\right\|_{a, \lambda}^{2}\right),
\end{aligned}
$$

with $\theta>0$.

Our first goal is to obtain the basic weighted energy estimates of $(\phi, \psi, \bar{\phi}, \bar{\psi})$.

Lemma 4.2. Under the same conditions in Proposition 4.1, then the solution $(\phi, \psi, \bar{\phi}, \bar{\psi})$ to the IBVP (3.3)-(3.7) satisfies for $t \in[0, T]$ that

$$
\begin{aligned}
& (1+t)^{\xi}\|(\phi, \psi, \bar{\phi}, \bar{\psi})\|_{a, \nu}^{2}+\nu \int_{0}^{t}(1+\tau)^{\xi}\|(\phi, \psi, \bar{\phi}, \bar{\psi})\|_{a, \nu-1}^{2} d \tau \\
& +\int_{0}^{t}(1+\tau)^{\xi}\left\|\left(\psi_{x}, \bar{\psi}_{x}, \bar{\psi}-\psi\right)\right\|_{a, \nu}^{2} d \tau+\int_{0}^{t}(1+\tau)^{\xi}\left(\phi^{2}(t, 0)+\bar{\phi}^{2}(t, 0)\right) d \tau \\
\leq & C\left\|\left(\phi_{0}, \psi_{0}, \bar{\phi}_{0}, \bar{\psi}_{0}\right)\right\|_{a, \lambda}^{2}+C \delta \int_{0}^{t}(1+\tau)^{\xi}\left\|\left(\phi_{x}, \bar{\phi}_{x}\right)\right\|^{2} d \tau \\
& +C \nu \int_{0}^{t}(1+\tau)^{\xi}\left\|\left(\psi_{x}, \bar{\psi}_{x}, \bar{\psi}-\psi\right)\right\|_{a, \nu-1}^{2} d \tau+C \xi \int_{0}^{t}(1+\tau)^{\xi-1}\|(\phi, \psi, \bar{\phi}, \bar{\psi})\|_{a, \nu}^{2} d \tau .
\end{aligned}
$$

with $\xi \geq 0$. 
Proof. We multiply (3.16) by $W_{a, \nu}$, where $W_{a, \nu}:=(1+x)^{\nu}$ is a space weight function. We integrate the resulted equality over $\mathbb{R}_{+}$to obtain

$$
\begin{aligned}
& \frac{d}{d t} \int W_{a, \nu}\left(\mathcal{E}_{1}+\mathcal{E}_{2}\right)-\left(W_{a, \nu} G_{1}\right)(t, 0)-\int W_{a, \nu-1} G_{1} d x-\int W_{a, \nu-1} G_{2} d x \\
& +\int W_{a, \nu}\left[\mu \psi_{x}^{2}+n \bar{\psi}_{x}^{2}+n(\bar{\psi}-\psi)^{2}\right] d x \\
& =-\int W_{a, \nu}\left(R_{1}+R_{2}+R_{3}\right) d x,
\end{aligned}
$$

where $\mathcal{E}_{i}, i=1,2$ are defined by (3.14)-(3.15), and $G_{j}$ for $j=1,2, R_{k}$ for $k=1,2,3$ are defined by (3.17). First, we estimate terms on the left hand side of (4.4). Under the condition (3.7), the second term on the left hand side is estimated as

$$
-\left(W_{a, \nu} G_{1}\right)(t, 0)=\left|u_{-}\right|\left[\Phi_{1}(\rho(t, 0), \widetilde{\rho}(0))+\Phi_{2}(n(t, 0), \widetilde{n}(0))\right] \geq c\left(\phi^{2}(t, 0)+\bar{\phi}^{2}(t, 0)\right),
$$

We decompose $\psi$ as $\psi=\bar{\psi}+(\psi-\bar{\psi})$ and use (1.11) to gain

$$
\begin{aligned}
& -\nu \int W_{a, \nu-1} G_{1} d x \\
\geq & \nu \int W_{a, \nu-1}\left[\frac{1}{2}(\phi, \bar{\phi}, \bar{\psi}) M_{3}(\phi, \bar{\phi}, \bar{\psi})^{\mathrm{T}}-\rho_{+} u_{+} \bar{\psi}(\psi-\bar{\psi})-A_{1} \gamma \rho_{+}^{\gamma-1} \phi(\psi-\bar{\psi})\right] d x \\
& -C(\delta+\varepsilon)\|(\phi, \psi, \bar{\phi}, \bar{\psi})\|_{a, \nu-1}^{2},
\end{aligned}
$$

where the symmetric matrix $\boldsymbol{M}_{\mathbf{3}}$ is denoted by

$$
M_{3}=\left(\begin{array}{ccc}
-A_{1} \gamma \rho_{+}^{\gamma-2} u_{+} & 0 & -A_{1} \gamma \rho_{+}^{\gamma-1} \\
0 & -A_{2} \alpha n_{+}^{\alpha-2} u_{+} & -A_{2} \alpha n_{+}^{\alpha-1} \\
-A_{1} \gamma \rho_{+}^{\gamma-1} & -A_{2} \alpha n_{+}^{\alpha-1} & -\left(\rho_{+}+n_{+}\right) u_{+}
\end{array}\right) .
$$

It is easy to verify that $M_{\mathbf{3}}$ is a positive definite matrix for $M_{+}>1$. Hence, the estimate of the third term on the left hand side is obtained under the condition $\varepsilon, \delta$ and $\eta$ small enough that

$$
\begin{aligned}
& -\nu \int W_{a, \nu-1} G_{1} d x \\
\geq & c \nu\|(\phi, \psi, \bar{\phi}, \bar{\psi})\|_{a, \nu-1}^{2}-\eta \nu\|(\phi, \bar{\psi})\|_{a, \nu-1}^{2}-C_{\eta} \nu\|\bar{\psi}-\psi\|_{a, \nu-1}^{2}-C(\varepsilon+\delta)\|(\phi, \psi, \bar{\phi}, \bar{\psi})\|_{a, \nu-1}^{2} \\
\geq & c \nu\|(\phi, \psi, \bar{\phi}, \bar{\psi})\|_{a, \nu-1}^{2}-C \nu\|\bar{\psi}-\psi\|_{a, \nu-1}^{2},
\end{aligned}
$$

By Young inequality with $0<\eta<1$, the forth and fifth terms on the left hand side are estimated as

$$
\begin{gathered}
-\nu \int W_{a, \nu-1} G_{2} d x \leq \nu \eta\|(\psi, \bar{\psi})\|_{a, \nu-1}^{2}+\nu C_{\eta}\left\|\left(\psi_{x}, \bar{\psi}_{x}\right)\right\|_{a, \nu-1}^{2}+C \delta\|(\bar{\phi}, \bar{\psi})\|_{a, \nu-1}^{2} . \\
\int W_{a, \nu}\left[\mu \psi_{x}^{2}+n \bar{\psi}_{x}^{2}+n(\psi-\bar{\psi})^{2}\right] d x \geq c\left\|\left(\psi_{x}, \bar{\psi}_{x}, \bar{\psi}-\psi\right)\right\|_{a, \nu}^{2}-C(\varepsilon+\delta)\left\|\left(\psi_{x}, \bar{\psi}_{x}, \psi-\bar{\psi}\right)\right\|_{a, \nu}^{2} .
\end{gathered}
$$

By (1.11) and (3.11), it follows from Sobolev inequality and Cauchy-Schwarz inequality that

$$
\begin{aligned}
\left|\int W_{a, \nu}\left(R_{1}+R_{2}+R_{3}\right) d x\right| & \leq C \delta \int e^{-\frac{c_{0}}{2} x}\left(\phi^{2}+\psi^{2}+\bar{\phi}^{2}+\bar{\psi}^{2}+|\bar{\psi}-\psi|^{2}\right) d x \\
& \leq C \delta\left\|\left(\phi_{x}, \psi_{x}, \bar{\phi}_{x}, \bar{\psi}_{x}, \bar{\psi}-\psi\right)\right\|^{2}+C \delta\left(\phi^{2}(t, 0)+\bar{\phi}^{2}(t, 0)\right) .
\end{aligned}
$$


Finally, with $\eta, \delta$ and $\varepsilon$ suitably small, the substitution of (4.5)-(4.11) into (4.4) leads to

$$
\begin{aligned}
& \frac{d}{d t} \int W_{a, \nu}\left(\mathcal{E}_{1}+\mathcal{E}_{2}\right) d x+c \nu\|(\phi, \psi, \bar{\phi}, \bar{\psi})\|_{a, \nu-1}^{2}+c\left\|\left(\psi_{x}, \bar{\psi}_{x}, \psi-\bar{\psi}\right)\right\|_{a, \nu}^{2}+c\left(\phi^{2}(t, 0)+\bar{\phi}^{2}(t, 0)\right) \\
\leq & C \delta\left\|\left(\phi_{x}, \bar{\phi}_{x}\right)\right\|^{2}+C \nu\left\|\left(\psi_{x}, \bar{\psi}_{x}, \psi-\bar{\psi}\right)\right\|_{a, \nu-1}^{2} .
\end{aligned}
$$

Multiplying (4.12) by $(1+\tau)^{\xi}$ and integrating the resulted equation in $\tau$ over $[0, t]$, we gain the desired estimate (4.3).

Similar to Lemmas 3.4-3.5, we get the following high order weighted estimates of $(\phi, \psi, \bar{\phi}, \bar{\psi})$. The details are omitted.

Lemma 4.3. Under the same conditions in Proposition 4.1, then the solution $(\phi, \psi, \bar{\phi}, \bar{\psi})$ to the IBVP (3.3)-(3.7) satisfies for $t \in[0, T]$ that

$$
\begin{aligned}
& (1+t)^{\xi}\left\|\left(\phi_{x}, \bar{\phi}_{x}\right)\right\|^{2}+\int_{0}^{t}(1+\tau)^{\xi}\left\|\left(\phi_{x}, \bar{\phi}_{x}\right)\right\|^{2} d \tau \\
\leq & C\left(\left\|\left(\phi_{0}, \psi_{0}, \bar{\phi}_{0}, \bar{\psi}_{0}\right)\right\|_{a, \lambda}^{2}+\left\|\left(\phi_{0 x}, \bar{\phi}_{0 x}\right)\right\|^{2}\right)+C \nu \int_{0}^{t}(1+\tau)^{\xi}\left\|\left(\psi_{x}, \bar{\psi}_{x}, \psi-\bar{\psi}\right)\right\|_{a, \nu-1}^{2} d \tau \\
& +C \varepsilon \int_{0}^{t}(1+\tau)^{\xi}\left\|\left(\psi_{x x}, \bar{\psi}_{x x}\right)\right\|_{a, \nu}^{2} d \tau+C \xi \int_{0}^{t}(1+\tau)^{\xi-1}\left(\|(\phi, \psi, \bar{\phi}, \bar{\psi})\|_{a, \nu}^{2}+\left\|\left(\phi_{x}, \bar{\phi}_{x}\right)\right\|^{2}\right) d \tau,
\end{aligned}
$$

with $\xi \geq 0$.

Lemma 4.4. Under the same conditions in Proposition 4.1 hold, then the solution $(\phi, \psi, \bar{\phi}, \bar{\psi})$ to the IBVP (3.3)-(3.7) satisfies for $t \in[0, T]$ that

$$
\begin{aligned}
& (1+t)^{\xi}\left\|\left(\psi_{x}, \bar{\psi}_{x}\right)\right\|^{2}+\int_{0}^{t}(1+\tau)^{\xi}\left\|\left(\psi_{x x}, \bar{\psi}_{x x}\right)\right\|^{2} d \tau \\
\leq & C\left(\left\|\left(\phi_{0}, \psi_{0}, \bar{\phi}_{0}, \bar{\psi}_{0}\right)\right\|_{a, \lambda}^{2}+\left\|\left(\phi_{0 x}, \bar{\phi}_{0 x}, \psi_{0 x}, \bar{\psi}_{0 x}\right)\right\|^{2}\right)+C \nu \int_{0}^{t}(1+\tau)^{\xi}\left\|\left(\psi_{x}, \bar{\psi}_{x}, \psi-\bar{\psi}\right)\right\|_{a, \nu-1}^{2} d \tau \\
& +C \xi \int_{0}^{t}(1+\tau)^{\xi-1}\left(\|(\phi, \psi, \bar{\phi}, \bar{\psi})\|_{a, \nu}^{2}+\left\|\left(\phi_{x}, \psi_{x}, \bar{\phi}_{x}, \bar{\psi}_{x}\right)\right\|^{2}\right) d \tau
\end{aligned}
$$

with $\xi \geq 0$.

Proof of Proposition 4.1 For $\nu \in[0, \lambda]$ and $\xi \geq 0$, it follows from Lemmas 4.2-4.4 that

$$
\begin{aligned}
& (1+t)^{\xi}\left(\|(\phi, \psi, \bar{\phi}, \bar{\psi})\|_{a, \nu}^{2}+\left\|\left(\phi_{x}, \psi_{x}, \bar{\psi}_{x}, \bar{\phi}_{x}\right)\right\|^{2}\right)+\nu \int(1+t)^{\xi}\|(\phi, \psi, \bar{\phi}, \bar{\psi})\|_{a, \nu-1}^{2} d \tau \\
& +\int_{0}^{t}(1+\tau)^{\xi}\left\|\left(\psi_{x}, \bar{\psi}_{x}, \psi-\bar{\psi}\right)\right\|_{a, \nu}^{2} d \tau+\int_{0}^{t}(1+\tau)^{\xi}\left\|\left(\phi_{x}, \psi_{x x}, \bar{\phi}_{x}, \bar{\psi}_{x x}\right)\right\|^{2} d \tau \\
\leq & C\left(\left\|\left(\phi_{0}, \psi_{0}, \bar{\phi}_{0}, \bar{\psi}_{0}\right)\right\|_{a, \lambda}^{2}+\left\|\left(\phi_{0 x}, \psi_{0 x}, \bar{\phi}_{0 x}, \bar{\psi}_{0 x}\right)\right\|^{2}\right)+C \nu \int_{0}^{t}(1+\tau)^{\xi}\left\|\left(\psi_{x}, \bar{\psi}_{x}, \bar{\psi}-\psi\right)\right\|_{a, \nu-1}^{2} d \tau \\
& +C \xi \int_{0}^{t}(1+\tau)^{\xi-1}\left(\|(\phi, \psi, \bar{\phi}, \bar{\psi})\|_{a, \nu}^{2}+\left\|\left(\phi_{x}, \psi_{x}, \bar{\psi}_{x}, \bar{\phi}_{x}\right)\right\|^{2}\right),
\end{aligned}
$$

where $C>0$ is a generic positive constant independent of $T, \nu$, and $\xi$. Hence, applying similar induction arguments as in $[3,14,25]$ to $(4.15)$, we gain the desired estimate (4.2). 
Indeed, for any $\lambda>0$ and $k=0,1,2, \ldots[\lambda]$, we have

$$
\begin{aligned}
& (1+t)^{k}\left(\|(\phi, \psi, \bar{\phi}, \bar{\psi})\|_{a, \lambda-k}^{2}+\left\|\left(\phi_{x}, \psi_{x}, \bar{\psi}_{x}, \bar{\phi}_{x}\right)\right\|^{2}\right)+\nu \int(1+t)^{k}\|(\phi, \psi, \bar{\phi}, \bar{\psi})\|_{a, \lambda-k-1}^{2} d \tau \\
& +\int_{0}^{t}(1+\tau)^{k}\left\|\left(\psi_{x}, \bar{\psi}_{x}, \psi-\bar{\psi}\right)\right\|_{a, \lambda-k}^{2} d \tau+\int_{0}^{t}(1+\tau)^{k}\left\|\left(\phi_{x}, \psi_{x x}, \bar{\phi}_{x}, \bar{\psi}_{x x}\right)\right\|^{2} d \tau \\
\leq & C\left(\left\|\left(\phi_{0}, \psi_{0}, \bar{\phi}_{0}, \bar{\psi}_{0}\right)\right\|_{a, \lambda}^{2}+\left\|\left(\phi_{0 x}, \psi_{0 x}, \bar{\phi}_{0 x}, \bar{\psi}_{0 x}\right)\right\|^{2}\right),
\end{aligned}
$$

and

$$
\begin{aligned}
& (1+t)^{k}\|(\phi, \psi, \bar{\phi}, \bar{\psi})\|_{1}^{2}+\int_{0}^{t}(1+\tau)^{k}\left\|\left(\phi_{x}, \psi_{x}, \psi_{x x}, \bar{\psi}_{x}, \bar{\phi}_{x}, \bar{\psi}_{x x}, \psi-\bar{\psi}\right)\right\|^{2} d \tau \\
\leq & C\left(\left\|\left(\phi_{0}, \psi_{0}, \bar{\phi}_{0}, \bar{\psi}_{0}\right)\right\|_{a, \lambda}^{2}+\left\|\left(\phi_{0 x}, \psi_{0 x}, \bar{\phi}_{0 x}, \bar{\psi}_{0 x}\right)\right\|^{2}\right) .
\end{aligned}
$$

To prove (4.16) and (4.17), we apply similar induction arguments as in [3,14,25] to (4.15).

Step 1. Taking $\xi=0, \nu=\lambda$ in (4.15) and using (3.9), we have (4.16) and (4.17) for $k=0$. Therefore, (4.16) and (4.17) hold for $0<\lambda<1$.

Step 2. Taking $\xi=1, \nu=0$ in (4.15) and using (4.16) with $k=0$, we have (4.17) with $k=1$. Then, taking $\xi=1, \nu=\lambda-1$ in (4.15) and using (4.17) with $k=1$ and (4.16) with $k=0$, we obtain the desired estimate (4.16) with $k=1$. Therefore, the proof is finished for $1 \leq \lambda<2$.

Step 3. We repeat the same procedure as in Step 2. The estimate (4.15) (with $\xi=2, \nu=0$ ) together with (4.17) (with $k=1$ ) lead to (4.17) (with $k=2$ ). Also, (4.15) (with $\xi=2, \nu=\lambda-2$ ) together with (4.17) (with $k=2$ ) and (4.16) (with $k=1$ ) lead to (4.16) (with $k=2$ ), which proves the estimates (4.16) and (4.17) for $2 \leq \lambda<3$.

Repeating the same procedure, we get the desired estimates (4.16) and (4.17) for any $\lambda>0$.

If $\lambda>0$ is integer, we obtain (4.2) from (4.16) letting $k=\lambda$.

If $\lambda>0$ is not integer, we obtain (4.2) as follows.

Taking $\nu=0$ in (4.15), we have

$$
\begin{aligned}
& (1+t)^{\xi}\|(\phi, \psi, \bar{\phi}, \bar{\psi})\|_{1}^{2}+\int_{0}^{t}(1+\tau)^{\xi}\left\|\left(\phi_{x}, \psi_{x}, \psi_{x x}, \bar{\phi}_{x}, \bar{\psi}_{x}, \bar{\psi}_{x x}, \psi-\bar{\psi}\right)\right\|^{2} d \tau \\
\leq & C\left(\left\|\left(\phi_{0}, \psi_{0}, \bar{\phi}_{0}, \bar{\psi}_{0}\right)\right\|_{a, \lambda}^{2}+\left\|\left(\phi_{0 x}, \psi_{0 x}, \bar{\phi}_{0 x}, \bar{\psi}_{0 x}\right)\right\|^{2}\right)+C \xi \int_{0}^{t}(1+\tau)^{\xi-1}\|(\phi, \psi, \bar{\phi}, \bar{\psi})\|_{1}^{2} d \tau .
\end{aligned}
$$

Using (4.16) with $k=[\lambda]$ and taking $s=1-(\lambda-[\lambda])$, we have

$$
\begin{aligned}
& \int_{0}^{t}(1+\tau)^{\xi-1}\|(\phi, \psi, \bar{\phi}, \bar{\psi})\|_{1}^{2} d \tau \\
\leq & \int_{0}^{t}(1+\tau)^{\xi-1-[\lambda]}\left\{\left((1+t)^{[\lambda]}\|(\phi, \psi, \bar{\phi}, \bar{\phi})\|_{a, \lambda-[\lambda]}^{2}\right)^{s}\left((1+t)^{[\lambda]}\|(\phi, \psi, \bar{\phi}, \bar{\phi})\|_{a, \lambda-[\lambda]-1}^{2}\right)^{1-s}\right. \\
& \left.+(1+t)^{[\lambda]}\left\|\left(\phi_{x}, \psi_{x}, \bar{\phi}_{x}, \bar{\psi}_{x}\right)\right\|^{2}\right\} d \tau \\
\leq & C\left(\left\|\left(\phi_{0}, \psi_{0}, \bar{\phi}_{0}, \bar{\psi}_{0}\right)\right\|_{a, \lambda}^{2}+\left\|\left(\phi_{0 x}, \psi_{0 x}, \bar{\phi}_{0 x}, \bar{\psi}_{0 x}\right)\right\|^{2}\right)^{s} \int_{0}^{t}(1+\tau)^{\xi-1-[\lambda]}\left((1+t)^{[\lambda]}\left\|\left(\phi_{x}, \psi_{x}, \bar{\phi}_{x}, \bar{\psi}_{x}\right)\right\|^{2}\right. \\
& \left.+(1+t)^{[\lambda]}\|(\phi, \psi, \bar{\phi}, \bar{\phi})\|_{a, \lambda-[\lambda]-1}^{2}\right)^{1-s} d \tau
\end{aligned}
$$




$$
\begin{aligned}
& \leq C\left(\left\|\left(\phi_{0}, \psi_{0}, \bar{\phi}_{0}, \bar{\psi}_{0}\right)\right\|_{a, \lambda}^{2}+\left\|\left(\phi_{0 x}, \psi_{0 x}, \bar{\phi}_{0 x}, \bar{\psi}_{0 x}\right)\right\|^{2}\right)\left(\int_{0}^{t}(1+\tau)^{\frac{\xi-1-[\lambda]}{1+[\lambda]-\lambda}} d \tau\right)^{1+[\lambda]-\lambda} \\
& \leq C\left(\left\|\left(\phi_{0}, \psi_{0}, \bar{\phi}_{0}, \bar{\psi}_{0}\right)\right\|_{a, \lambda}^{2}+\left\|\left(\phi_{0 x}, \psi_{0 x}, \bar{\phi}_{0 x}, \bar{\psi}_{0 x}\right)\right\|^{2}\right)(1+t)^{\theta}
\end{aligned}
$$

where we take $\xi=\lambda+\theta(1+[\lambda]-\lambda)$ and $\theta>0$.

\subsection{Convergence rate of sonic steady-state}

The function space $Y_{W}(0, T)$ for $T>0$ is denoted by

$$
\begin{aligned}
Y_{W}(0, T):=\{(\phi, \psi, \bar{\phi}, \bar{\psi}) \mid & (\phi, \psi, \bar{\phi}, \bar{\psi}) \in C\left([0, T] ; H_{W}^{1}\left(\mathbb{R}_{+}\right)\right), \\
& \left.\left(\phi_{x}, \bar{\phi}_{x}\right) \in L^{2}\left([0, T] ; L_{W}^{2}\left(\mathbb{R}_{+}\right)\right),\left(\psi_{x}, \bar{\psi}_{x}\right) \in L^{2}\left([0, T] ; H_{W}^{1}\left(\mathbb{R}_{+}\right)\right)\right\} .
\end{aligned}
$$

Proposition 4.5. Assume that $1 \leq \lambda<\lambda^{*}$ with $\lambda^{*}:=2+\sqrt{8+\frac{1}{1+b^{2}}}, b:=\frac{\rho_{+}\left(u_{+}^{2}-p_{1}^{\prime}\left(\rho_{+}\right)\right)}{\left|u_{+}\right| \sqrt{\left(\mu+n_{+}\right) n_{+}}}$, and that the same conditions in Theorem 1.3 hold for $M_{+}=1$. Let $(\phi, \psi, \bar{\phi}, \bar{\psi})$ be a solution to the IBVP (3.3)-(3.7) satisfying $(\phi, \psi, \bar{\phi}, \bar{\psi}) \in Y_{\sigma^{-\lambda}}(0, T)$ for any time $T>0$. Then for arbitrary $\nu \in(0, \lambda]$, there exist positive constants $\varepsilon>0$ and $C>0$ independent of $T$ such that if

$$
\sup _{0 \leq t \leq T}\left\|\sigma^{-\frac{\lambda}{2}}(\phi, \psi, \bar{\phi}, \bar{\psi})(t)\right\|_{1}+\delta^{\frac{1}{2}} \leq \varepsilon
$$

is satisfied, it holds for arbitrary $t \in[0, T]$ that

$$
\begin{aligned}
& \quad(1+\delta t)^{\frac{\lambda-\nu}{2}+\beta}\left\|\sigma^{-\frac{\nu}{2}}(\phi, \psi, \bar{\phi}, \bar{\psi})\right\|_{1}^{2}+\int_{0}^{t}(1+\delta \tau)^{\frac{\lambda-\nu}{2}+\beta}\left\|\sigma^{-\frac{\nu-2}{2}}(\phi, \psi, \bar{\phi}, \bar{\psi})\right\|^{2} d \tau \\
& \quad+\int_{0}^{t}(1+\delta \tau)^{\frac{\lambda-\nu}{2}+\beta}\left\|\sigma^{-\frac{\nu}{2}}\left(\phi_{x}, \psi_{x}, \bar{\phi}_{x}, \bar{\psi}_{x}\right)\right\|^{2} d \tau+\int_{0}^{t}(1+\delta \tau)^{\frac{\lambda-\nu}{2}+\beta}\left\|\sigma^{-\frac{\nu}{2}}\left(\psi_{x x}, \bar{\psi}_{x x}, \bar{\psi}-\psi\right)\right\|^{2} d \tau \\
& \leq C(1+\delta t)^{\beta}\left\|\sigma^{-\frac{\lambda}{2}}\left(\phi_{0}, \psi_{0}, \phi_{0 x}, \psi_{0 x}, \bar{\phi}_{0}, \bar{\psi}_{0}, \bar{\phi}_{0 x}, \bar{\psi}_{0 x}\right)\right\|^{2}, \\
& \text { with } \beta>0 .
\end{aligned}
$$

By the fact $\lambda \geq 1$ and (4.21), it is easy to verify the following estimate:

$$
\left\|\sigma^{-\frac{1}{2}}(\phi, \psi, \bar{\phi}, \bar{\psi})\right\|_{L^{\infty}} \leq\left\|\sigma^{-\frac{\lambda}{2}}(\phi, \psi, \bar{\phi}, \bar{\psi})\right\|_{1} \leq \sqrt{2} \varepsilon
$$

To deal with some nonlinear terms, we use the following inequality as in $[15,27,36]$.

Lemma 4.6 ( $[36])$. Let $\nu \geq 1$. Then a function $\sigma^{-\frac{\nu}{2}}(x) \phi(t, x) \in H^{1}\left(\mathbb{R}_{+}\right)$satisfies

$$
\int \sigma^{-\frac{\nu-1}{2}}|\phi|^{3} d x \leq C\left\|\sigma^{-\frac{1}{2}} \phi\right\|\left(\sigma(0) \phi^{2}(t, 0)+\left\|\sigma^{-\frac{\nu}{2}} \phi_{x}\right\|^{2}+\left\|\sigma^{-\frac{\nu-2}{2}} \phi\right\|^{2}\right),
$$

where the function $\sigma(x) \geq 0$ is defined by (2.28) with $\sigma(0)$ small enough.

To gain faster decay rates, it is necessary to use the following Hardy type inequality. 
Lemma 4.7 ( [13] ). Let $\zeta \in C^{1}[0, \infty)$ satisfies $\zeta>0, \zeta_{x}>0$ and $\zeta(x) \rightarrow \infty$ for $x \rightarrow \infty$. Then we have

$$
\int_{\mathbb{R}_{+}} \psi^{2} \zeta_{x} d x \leq 4 \int_{\mathbb{R}_{+}} \psi_{x}^{2} \frac{\zeta^{2}}{\zeta_{x}} d x
$$

for $\psi$ satisfying $\psi(t, 0)=0$ and $\sqrt{w} \psi \in H^{1}\left(\mathbb{R}_{+}\right)$, with the function $w:=\frac{\zeta^{2}}{\zeta_{x}}$.

With the aid of Lemmas 4.6-4.7, we obtain the weighted $L^{2}$ estimate of $(\phi, \psi, \bar{\phi}, \bar{\psi})$.

Lemma 4.8. Under the same conditions in Proposition 4.5, then the solution $(\phi, \psi, \bar{\phi}, \bar{\psi})$ to the problem (3.3)-(3.7) satisfies for $t \in[0, T]$ that

$$
\begin{aligned}
& \quad(1+\delta \tau)^{\xi}\left\|\sigma^{-\frac{\nu}{2}}(\phi, \psi, \bar{\phi}, \bar{\psi})\right\|^{2}+\int_{0}^{t}(1+\delta \tau)^{\xi}\left\|\sigma^{-\frac{\nu-2}{2}}(\phi, \psi, \bar{\phi}, \bar{\psi})\right\|^{2} d \tau \\
& \quad+\int_{0}^{t}(1+\delta \tau)^{\xi}\left\|\sigma^{-\frac{\nu}{2}}\left(\psi_{x}, \bar{\psi}_{x}, \bar{\psi}-\psi\right)\right\|^{2} d \tau+\int_{0}^{t}(1+\delta \tau)^{\xi} \frac{1}{\delta^{\nu}}\left(\phi^{2}(t, 0)+\bar{\phi}^{2}(t, 0)\right) d \tau \\
& \leq C\left\|\sigma^{-\frac{\lambda}{2}}\left(\phi_{0}, \psi_{0}, \bar{\phi}_{0}, \bar{\psi}_{0}\right)\right\|^{2}+C \delta \int_{0}^{t}(1+\delta \tau)^{\xi}\left\|\sigma^{-\frac{\nu}{2}}\left(\phi_{x}, \bar{\phi}_{x}\right)\right\|^{2} d \tau \\
& \quad+C \delta \xi \int_{0}^{t}(1+\delta \tau)^{\xi-1}\left\|\sigma^{-\frac{\nu}{2}}(\phi, \psi, \bar{\phi}, \bar{\psi})\right\|^{2} d \tau,
\end{aligned}
$$

with $\xi \geq 0$.

Proof. We multiply (3.16) by the space weight function $\sigma^{-\nu}$, where the space weight function $\sigma \geq 0$ satisfies (1.14) and (2.28). Then, we integrate the resulted equation over $\mathbb{R}_{+}$to get

$$
\begin{aligned}
& \frac{d}{d t} \int \sigma^{-\nu}\left(\mathcal{E}_{1}+\mathcal{E}_{2}\right) d x-\left(\sigma^{-\nu} G_{1}\right)(t, 0)-a \nu \int \sigma^{-(\nu-1)} G_{1} d x-a \nu \int \sigma^{-(\nu-1)} G_{2} d x \\
& +\int \sigma^{-\nu} n(\bar{\psi}-\psi)^{2} d x+\int \sigma^{-\nu}\left(\mu \psi_{x}^{2}+n \bar{\psi}_{x}^{2}\right) d x+\int \sigma^{-\nu} R_{1} d x \\
& =-\int \sigma^{-\nu} R_{2} d x-\int \sigma^{-\nu} R_{3} d x,
\end{aligned}
$$

where $\mathcal{E}_{i}, i=1,2$ are defined by (3.14)-(3.15), and $G_{j}$ for $j=1,2, R_{k}$ for $k=1,2,3$ are defined by (3.17). First, we estimate terms on the left hand side of (4.27). Under the condition (3.7), the second term on the left hand side is estimated as

$$
-\left(\sigma^{-\nu} G_{1}\right)(t, 0) \geq \frac{c}{\delta^{\nu}}\left(\phi^{2}(t, 0)+\bar{\phi}^{2}(t, 0)\right) \geq 0 .
$$

For the third term on the left hand side, using (4.24) and $\psi=\bar{\psi}+(\psi-\bar{\psi})$ yields

$$
\begin{aligned}
& -a \nu \int \sigma^{-(\nu-1)} G_{1} d x \\
\geq & a \nu \int \sigma^{-(\nu-1)}\left[\frac{1}{2}(\phi, \bar{\phi}, \bar{\psi}) M_{4}(\phi, \bar{\phi}, \bar{\psi})^{\mathrm{T}}-\rho_{+} u_{+} \bar{\psi}(\psi-\bar{\psi})-A_{1} \gamma \rho_{+}^{\gamma-1} \phi(\psi-\bar{\psi})\right] d x \\
& +a \nu \int \sigma^{-(\nu-1)}\left[-\left(A_{1} \gamma \widetilde{\rho}^{\gamma-2} \widetilde{u}-A_{1} \gamma \widetilde{\rho}_{+}^{\gamma-2} u_{+}\right) \frac{\phi^{2}}{2}-\left(A_{2} \alpha \widetilde{n}^{\alpha-2} \widetilde{v}-A_{2} \alpha n_{+}^{\alpha-2} u_{+}\right) \frac{\bar{\phi}^{2}}{2}\right. \\
& \left.-\left(A_{1} \gamma \widetilde{\rho}^{\gamma-1}-A_{1} \gamma \rho_{+}^{\gamma-1}\right) \phi \psi-\left(A_{2} \alpha \widetilde{n}^{\alpha-1}-A_{2} \alpha n_{+}^{\alpha-1}\right) \bar{\phi} \bar{\psi}\right] d x-C \frac{\varepsilon}{\delta^{\nu}}\left(\phi^{2}(t, 0)+\bar{\phi}^{2}(t, 0)\right) \\
& -C \varepsilon\left(\left\|\sigma^{-\left(\frac{\nu-2}{2}\right)}(\phi, \psi, \bar{\phi}, \bar{\psi})\right\|^{2}+\left\|\sigma^{-\frac{\nu}{2}}\left(\phi_{x}, \psi_{x}, \bar{\phi}_{x}, \bar{\psi}_{x}\right)\right\|^{2}\right),
\end{aligned}
$$


where the symmetric matrix $\boldsymbol{M}_{\mathbf{4}}$ is defined as

$$
\boldsymbol{M}_{4}=\left(\begin{array}{ccc}
-A_{1} \gamma \rho_{+}^{\gamma-2} u_{+} & 0 & -A_{1} \gamma \rho_{+}^{\gamma-1} \\
0 & -A_{2} \alpha n_{+}^{\alpha-2} u_{+} & -A_{2} \alpha n_{+}^{\alpha-1} \\
-A_{1} \gamma \rho_{+}^{\gamma-1} & -A_{2} \alpha n_{+}^{\alpha-1} & -\left(\rho_{+}+n_{+}\right) u_{+}
\end{array}\right) .
$$

Owing to $M_{+}=1$, it is easy to check that three eigenvalues of the matrix $\boldsymbol{M}_{\mathbf{4}}$ satisfy: $\hat{\lambda}_{1}>0, \hat{\lambda}_{2}>0$, $\hat{\lambda}_{3}=0$. Take the coordinate transformation

$$
\left(\begin{array}{l}
\phi \\
\bar{\phi} \\
\bar{\psi}
\end{array}\right)=\boldsymbol{P}\left(\begin{array}{l}
\hat{\rho} \\
\hat{n} \\
\hat{v}
\end{array}\right)
$$

where the matrix $\boldsymbol{P}$ is denoted by

$$
\boldsymbol{P}=\left(\begin{array}{ccc}
r_{11} & r_{21} & -\frac{\rho_{+}}{u_{+}} \\
r_{12} & r_{22} & -\frac{n_{+}}{u_{+}} \\
r_{13} & r_{23} & 1
\end{array}\right) \text { with constants } r_{i j} \text { for } 1 \leq i \leq 2,1 \leq j \leq 3,
$$

such that

$$
(\phi, \bar{\phi}, \bar{\psi}) \boldsymbol{M}_{\mathbf{4}}(\phi, \bar{\phi}, \bar{\psi})^{\mathrm{T}}=(\hat{\rho}, \hat{n}, \hat{v})\left(\begin{array}{ccc}
\hat{\lambda}_{1} & 0 & 0 \\
0 & \hat{\lambda}_{2} & 0 \\
0 & 0 & 0
\end{array}\right)(\hat{\rho}, \hat{n}, \hat{v})^{\mathrm{T}}=\hat{\lambda}_{1} \hat{\rho}^{2}+\hat{\lambda}_{2} \hat{n}^{2}
$$

By (1.13), (4.23), (4.29), and (4.31)-(4.33), the third term is estimated as

$$
\begin{aligned}
& -a \nu \int \sigma^{-(\nu-1)} G_{1} d x \\
\geq & a \nu \int \sigma^{-(\nu-1)}\left(\frac{\hat{\lambda}_{1}}{2} \hat{\rho}^{2}+\frac{\hat{\lambda}_{2}}{2} \hat{n}^{2}\right) d x+a \nu \int \sigma^{-(\nu-2)} \frac{A_{1} \gamma(\gamma+1) \rho_{+}^{\gamma}+A_{2} \alpha(\alpha+1) n_{+}^{\alpha}}{2\left|u_{+}\right|^{2}} \hat{v}^{2} d x \\
& +a \nu \int \sigma^{-(\nu-1)} \frac{\rho_{+}\left(u_{+}^{2}-A_{1} \gamma \rho_{+}^{\gamma-1}\right)}{\left|u_{+}\right|} \hat{v}(\psi-\bar{\psi}) d x-C \delta^{\frac{1}{2}}\left\|\sigma^{-\frac{\nu-1}{2}}(\hat{\rho}, \hat{n})\right\|^{2}-C \delta^{\frac{1}{2}}\left\|\sigma^{-\frac{\nu-2}{2}} \hat{v}\right\|^{2} \\
& -C \delta^{\frac{1}{2}}\left\|\sigma^{-\frac{\nu}{2}}(\psi-\bar{\psi})\right\|^{2}-C(\varepsilon+\delta)\left\|\sigma^{-\frac{\nu-2}{2}}(\phi, \psi, \bar{\phi}, \bar{\psi})\right\|^{2}-C \varepsilon\left\|\sigma^{-\frac{\nu}{2}}\left(\phi_{x}, \psi_{x}, \bar{\phi}_{x}, \bar{\psi}_{x}\right)\right\|^{2} \\
& -C \varepsilon \frac{1}{\delta^{\nu}}\left(\phi^{2}(t, 0)+\bar{\phi}^{2}(t, 0)\right),
\end{aligned}
$$

where we have used the following facts

$$
\begin{aligned}
& -\left(A_{1} \gamma \widetilde{\rho}^{\gamma-1}-A_{1} \gamma \rho_{+}^{\gamma-1}\right) \geq \frac{A_{1} \gamma(\gamma-1) \rho_{+}^{\gamma-1}}{\left|u_{+}\right|} \sigma-C \sigma^{2}, \\
& -\left(A_{2} \alpha \widetilde{n}^{\alpha-1}-A_{2} \alpha n_{+}^{\alpha-1}\right) \geq \frac{A_{2} \alpha(\alpha-1) n_{+}^{\alpha-1}}{\left|u_{+}\right|} \sigma-C \sigma^{2}, \\
& -\left(A_{1} \gamma \widetilde{\rho}^{\gamma-2} \widetilde{u}-A_{1} \gamma \rho_{+}^{\gamma-2} u_{+}\right) \geq A_{1} \gamma(3-\gamma) \rho_{+}^{\gamma-2} \sigma-C \sigma^{2}, \\
& -\left(A_{2} \alpha \widetilde{n}^{\alpha-2} \widetilde{v}-A_{2} \alpha n_{+}^{\alpha-2} u_{+}\right) \geq A_{2} \alpha(3-\alpha) n_{+}^{\alpha-2} \sigma-C \sigma^{2} .
\end{aligned}
$$


With the help of (1.12)-(1.14), (3.7), (4.21), (4.23)-(4.24), (4.31)-(4.33), and $\psi=\bar{\psi}+(\psi-\bar{\psi})$, it holds that

$$
\begin{aligned}
& -a \nu \int \sigma^{-(\nu-1)} G_{2}(t, x) d x \\
& \geq-a^{2} \frac{\mu+n_{+}}{2} \nu(\nu-1)\left\|\sigma^{-\frac{\nu-2}{2}} \bar{\psi}\right\|^{2}-C(\delta+\varepsilon)\left\|\sigma^{-\frac{\nu-2}{2}} \bar{\psi}\right\|^{2}-C \varepsilon\left\|\sigma^{-\frac{\nu}{2}} \bar{\psi}_{x}\right\|^{2}-C \delta\left\|\sigma^{-\frac{\nu}{2}}(\bar{\psi}-\psi)\right\|^{2} \\
& \geq-a^{2} \frac{\mu+n_{+}}{2} \nu(\nu-1)\left\|\sigma^{-\frac{\nu-2}{2}} \hat{v}\right\|^{2}-C \delta^{\frac{1}{2}}\left(\left\|\sigma^{-\frac{\nu-1}{2}}(\hat{\rho}, \hat{n})\right\|^{2}+\left\|\sigma^{-\frac{\nu-2}{2}} \hat{v}\right\|^{2}\right)-C(\delta+\varepsilon)\left\|\sigma^{-\frac{\nu-2}{2}} \bar{\psi}\right\|^{2} \\
& -C \varepsilon\left\|\sigma^{-\frac{\nu}{2}} \bar{\psi}_{x}\right\|^{2}-C \delta\left\|\sigma^{-\frac{\nu}{2}}(\bar{\psi}-\psi)\right\|^{2}, \\
& \int \sigma^{-\nu} n(\bar{\psi}-\psi)^{2} d x \geq\left[n_{+}-C(\delta+\varepsilon)\right]\left\|\sigma^{-\frac{\nu}{2}}(\bar{\psi}-\psi)\right\|^{2}, \\
& \quad \int \sigma^{-\nu} R_{1} d x \\
& \geq a \int \sigma^{-(\nu-2)}\left[\frac{A_{1} \gamma(\gamma-1) \rho_{+}^{\gamma-2}}{2} \phi^{2}+\rho_{+} \psi^{2}+\frac{A_{2} \alpha(\alpha-1) n_{+}^{\alpha-2}}{2} \bar{\phi}^{2}+n_{+} \bar{\psi}^{2}\right] d x \\
& \quad-C(\delta+\varepsilon)\left\|\sigma^{-\frac{\nu-2}{2}}(\phi, \psi, \bar{\phi}, \bar{\psi})\right\|^{2} \\
& \geq a \frac{A_{1} \gamma(\gamma+1) \rho_{+}^{\gamma}+A_{2} \alpha(\alpha+1) n_{+}^{\alpha}}{2\left|u_{+}\right|^{2}}\left\|\sigma^{-\frac{\nu-2}{2}} \hat{v}\right\|^{2}-C \delta\left(\left\|\sigma^{-\frac{\nu-1}{2}}(\hat{\rho}, \hat{n})\right\|^{2}+\left\|\sigma^{-\frac{\nu-2}{2}} \hat{v}\right\|^{2}\right) \\
& \quad-C(\delta+\varepsilon)\left\|\sigma^{-\frac{\nu-2}{2}}(\phi, \psi, \bar{\phi}, \bar{\psi})\right\|^{2} .
\end{aligned}
$$

For $\nu \in(0,3]$, with the help of (1.13) and (2.22), we add (4.34)-(4.37) together to have

$$
\begin{aligned}
& -a \nu \int \sigma^{-(\nu-1)} G_{1} d x-a \nu \int \sigma^{-(\nu-1)} G_{2} d x+\int \sigma^{-\nu} n(\bar{\psi}-\psi)^{2} d x+\int \sigma^{-\nu} R_{1} d x \\
& \geq c\left\|\sigma^{-\frac{\nu-1}{2}}(\hat{\rho}, \hat{n})\right\|^{2}+\frac{1}{4}\left\{a \frac{A_{1} \gamma(\gamma+1) \rho_{+}^{\gamma}+A_{2} \alpha(\alpha+1) n_{+}^{\alpha}}{2\left|u_{+}\right|^{2}}\left[1+\nu-\frac{\nu(\nu-1)}{2\left(1+b^{2}\right)}\right]\left\|\sigma^{-\frac{\nu-2}{2}} \hat{v}\right\|^{2}\right. \\
& \left.\quad+n_{+}\left\|\sigma^{-\frac{\nu}{2}}(\psi-\bar{\psi})\right\|^{2}\right\}+\int \sigma^{-\nu}(\psi-\bar{\psi}, \hat{v}) M_{5}(\psi-\bar{\psi}, \hat{v})^{\mathrm{T}} d x-C \delta^{\frac{1}{2}}\left\|\sigma^{-\frac{\nu-1}{2}}(\hat{\rho}, \hat{n})\right\|^{2} \\
& \quad-C \delta^{\frac{1}{2}}\left\|\sigma^{-\frac{\nu-2}{2}} \hat{v}\right\|^{2}-C\left(\varepsilon+\delta^{\frac{1}{2}}\right)\left\|\sigma^{-\frac{\nu}{2}}(\psi-\bar{\psi})\right\|^{2}-C(\varepsilon+\delta)\left\|\sigma^{-\frac{\nu}{2}}\left(\phi_{x}, \psi_{x}, \bar{\phi}_{x}, \bar{\psi}_{x}\right)\right\|^{2} \\
& \quad-C \frac{\varepsilon}{\delta^{\nu}}\left(\phi^{2}(t, 0)+\bar{\phi}^{2}(t, 0)\right)-C(\varepsilon+\delta)\left\|\sigma^{-\frac{\nu-2}{2}}(\phi, \psi, \bar{\phi}, \bar{\psi})\right\|^{2} \\
& \geq c\left\|\sigma^{-\frac{\nu-2}{2}}(\phi, \psi, \bar{\phi}, \bar{\psi})\right\|^{2}+c\left\|\sigma^{-\frac{\nu-1}{2}}(\hat{\rho}, \hat{n})\right\|^{2}+c\left\|\sigma^{-\frac{\nu}{2}}(\bar{\psi}-\psi)\right\|^{2} \\
& \quad-C(\delta+\varepsilon)\left\|\sigma^{-\frac{\nu}{2}}\left(\phi_{x}, \psi_{x}, \bar{\phi}_{x}, \bar{\psi}_{x}\right)\right\|^{2}-C \varepsilon \frac{\phi^{2}(t, 0)+\bar{\phi}^{2}(t, 0)}{\delta^{\nu}}
\end{aligned}
$$

where the positive definite matrix $\boldsymbol{M}_{\mathbf{5}}$ is defined by

$$
M_{\mathbf{5}}=\left(\begin{array}{cc}
\frac{3}{4} n_{+} & \frac{\sqrt{\left(\mu+n_{+}\right) n_{+}}}{2} a b \nu \sigma \\
\frac{\sqrt{\left(\mu+n_{+}\right) n_{+}}}{2} a b \nu \sigma & \frac{3}{4} a \frac{A_{1} \gamma(\gamma+1) \rho_{+}^{\gamma}+A_{2} \alpha(\alpha+1) n_{+}^{\alpha}}{2\left|u_{+}\right|^{2}}\left[(1+\nu)-\frac{\nu(\nu-1)}{2\left(1+b^{2}\right)}\right] \sigma^{2}
\end{array}\right) .
$$

Then, we consider the case $\nu \in\left[3,2+\sqrt{8+\frac{1}{1+b^{2}}}\right)$ using the Lemma 4.7 with $\zeta=\sigma^{-(\nu-1)}$. Therefore, 
with the aid of (2.22), the sixth term is estimated as below:

$$
\begin{aligned}
& \int \sigma^{-\nu}\left(\mu \psi_{x}^{2}+n \bar{\psi}_{x}^{2}\right) d x \\
\geq & a^{2}\left(\mu+n_{+}\right) \frac{(\nu-1)^{2}}{4}\left\|\sigma^{-\frac{\nu-2}{2}} \hat{v}\right\|^{2}-C \delta^{\frac{1}{2}}\left(\left\|\sigma^{-\frac{\nu-2}{2}} \hat{v}\right\|^{2}+\left\|\sigma^{-\frac{\nu-1}{2}}(\hat{\rho}, \hat{n})\right\|^{2}\right) \\
& -C(\delta+\varepsilon)\left\|\sigma^{-\frac{\nu}{2}}\left(\bar{\psi}-\psi, \bar{\psi}_{x}\right)\right\|^{2} .
\end{aligned}
$$

For $\nu \in(3, \lambda]$, adding (4.38) to (4.40), taking $k=\nu B\left[4\left(1+b^{2}\right) \nu+4 b^{2}+5-\nu^{2}\right]^{-\frac{1}{2}} \in(0,1)$, and using $(2.22)$ and $c|(\phi, \bar{\phi}, \bar{\psi})| \leq|(\hat{\rho}, \hat{n}, \hat{v})| \leq C|(\phi, \bar{\phi}, \bar{\psi})|$, we have

$$
\begin{aligned}
& \quad-a \nu \int \sigma^{-(\nu-1)} G_{1} d x-a \nu \int \sigma^{-(\nu-1)} G_{2} d x+\int \sigma^{-\nu} n(\bar{\psi}-\psi)^{2} d x+\int \sigma^{-\nu} R_{1} d x \\
& \quad+\int \sigma^{-\nu}\left(\mu \psi_{x}^{2}+n \bar{\psi}_{x}^{2}\right) d x \\
& \geq c\left\|\sigma^{-\frac{\nu-1}{2}}(\hat{\rho}, \hat{n})\right\|^{2}+\int \sigma^{-\nu}(\psi-\bar{\psi}, \hat{v}) M_{6}(\psi-\bar{\psi}, \hat{v})^{\mathrm{T}} d x+(1-k)\left\{n_{+}\left\|\sigma^{-\frac{\nu}{2}}(\psi-\bar{\psi})\right\|^{2}\right. \\
& \left.\quad+a \frac{A_{1} \gamma(\gamma+1) \rho_{+}^{\gamma}+A_{2} \alpha(\alpha+1) n_{+}^{\alpha}}{2\left|u_{+}\right|^{2}}\left[1+\nu-\frac{\nu(\nu-1)}{2\left(1+b^{2}\right)}+\frac{(\nu-1)^{2}}{4\left(1+b^{2}\right)}\right]\left\|\sigma^{-\frac{\nu-2}{2}} \hat{v}\right\|^{2}\right\} \\
& \quad-C \delta^{\frac{1}{2}}\left(\left\|\sigma^{-\frac{\nu-1}{2}}(\hat{\rho}, \hat{n})\right\|^{2}+\left\|\sigma^{-\frac{\nu-2}{2}} \hat{v}\right\|^{2}\right)-C(\varepsilon+\delta)\left\|\sigma^{-\frac{\nu}{2}}\left(\phi_{x}, \psi_{x}, \bar{\phi}_{x}, \bar{\psi}_{x}\right)\right\|^{2} \\
& \quad-C\left(\varepsilon+\delta^{\frac{1}{2}}\right)\left\|\sigma^{-\frac{\nu}{2}}(\psi-\bar{\psi})\right\|^{2}-C \frac{\varepsilon}{\delta^{\nu}}\left(\phi^{2}(t, 0)+\bar{\phi}^{2}(t, 0)\right)-C(\varepsilon+\delta)\left\|\sigma^{-\frac{\nu-2}{2}}(\phi, \psi, \bar{\phi}, \bar{\psi})\right\|^{2} \\
& \geq c\left\|\sigma^{-\frac{\nu-2}{2}}(\phi, \psi, \bar{\phi}, \bar{\psi})\right\|^{2}+c\left\|\sigma^{-\frac{\nu-1}{2}}(\hat{\rho}, \hat{n})\right\|^{2}+c\left\|\sigma^{-\frac{\nu}{2}}\left(\bar{\psi}-\psi, \psi_{x}, \bar{\psi}_{x}\right)\right\|^{2} \\
& \quad-C(\delta+\varepsilon)\left\|\sigma^{-\frac{\nu}{2}}\left(\phi_{x}, \bar{\phi}_{x}\right)\right\|^{2}-C \frac{\varepsilon}{\delta^{\nu}}\left(\phi^{2}(t, 0)+\bar{\phi}^{2}(t, 0)\right),
\end{aligned}
$$

where $\delta^{\frac{1}{2}}$ and $\varepsilon$ are small enough, and the positive definite matrix $\boldsymbol{M}_{\mathbf{6}}$ is defined as

$$
\boldsymbol{M}_{\mathbf{6}}=\left(\begin{array}{cc}
\frac{k n_{+}}{\frac{\sqrt{\left(\mu+n_{+}\right) n_{+}}}{2}} a b \nu \sigma \\
\frac{\sqrt{\left(\mu+n_{+}\right) n_{+}}}{2} a b \nu \sigma & k a \frac{A_{1} \gamma(\gamma+1) \rho_{+}^{\gamma}+A_{2} \alpha(\alpha+1) n_{+}^{\alpha}}{2\left|u_{+}\right|^{2}}\left[1+\nu-\frac{\nu(\nu-1)}{2\left(1+b^{2}\right)}+\frac{(\nu-1)^{2}}{4\left(1+b^{2}\right)}\right] \sigma^{2}
\end{array}\right) .
$$

By (1.12)-(1.13), (4.31)-(4.33), Cauchy-Schwarz inequality and $M_{+}=1$, we estimate terms on the right hand side as

$$
\begin{aligned}
& \left|\int \sigma^{-\nu} R_{2} d x+\int \sigma^{-\nu} R_{3} d x\right| \\
\leq & C \delta^{\frac{1}{2}}\left\|\sigma^{-\frac{\nu-1}{2}}(\hat{\rho}, \hat{n})\right\|^{2}+C \delta^{\frac{1}{2}}\left\|\sigma^{-\frac{\nu-2}{2}}(\phi, \psi, \bar{\phi}, \bar{\psi})\right\|^{2}+C \delta\left\|\sigma^{-\frac{\nu}{2}}\left(\bar{\psi}_{x}, \bar{\psi}-\psi\right)\right\|^{2} .
\end{aligned}
$$

Finally, taking $\delta^{\frac{1}{2}}$ and $\varepsilon$ small enough, and combining (4.28)-(4.43), we obtain

$$
\begin{aligned}
& \frac{d}{d t} \int \sigma^{-\nu}\left(\mathcal{E}_{1}+\mathcal{E}_{2}\right) d x+c\left\|\sigma^{-\frac{\nu-2}{2}}(\phi, \psi, \bar{\phi}, \bar{\psi})\right\|^{2}+c\left\|\sigma^{-\frac{\nu}{2}}\left(\psi_{x}, \bar{\psi}_{x}, \bar{\psi}-\psi\right)\right\|^{2}+\frac{c}{\delta^{\nu}}\left[\phi^{2}(t, 0)+\bar{\phi}^{2}(t, 0)\right] \\
\leq & C(\delta+\varepsilon)\left\|\sigma^{-\frac{\nu}{2}}\left(\phi_{x}, \bar{\phi}_{x}\right)\right\|^{2} .
\end{aligned}
$$

Multiplying (4.44) by $(1+\delta \tau)^{\xi}$ and integrating the resulted equation in $\tau$ over $[0, t]$, we obtain (4.26). The proof of Lemma 4.8 is completed. 
In order to show Proposition 4.5, we need to obtain the high order weighted estimates of $(\phi, \psi, \bar{\phi}, \bar{\psi})$.

Lemma 4.9. Under the same conditions in Proposition 4.5, then the solution $(\phi, \psi, \bar{\phi}, \bar{\psi})$ to the IBVP (3.3)-(3.7) satisfies for $t \in[0, T]$ that

$$
\begin{aligned}
& (1+\delta t)^{\xi}\left\|\sigma^{-\frac{\nu}{2}}\left(\phi_{x}, \bar{\phi}_{x}\right)\right\|^{2}+\int_{0}^{t}(1+\delta \tau)^{\xi}\left\|\sigma^{-\frac{\nu}{2}}\left(\phi_{x}, \bar{\phi}_{x}\right)\right\|^{2} d \tau \\
\leq & C\left(\left\|\sigma^{-\frac{\lambda}{2}}\left(\phi_{0}, \psi_{0}, \bar{\phi}_{0}, \bar{\psi}_{0}\right)\right\|^{2}+\left\|\sigma^{-\frac{\lambda}{2}}\left(\phi_{0 x}, \bar{\phi}_{0 x}\right)\right\|^{2}\right)+C(\varepsilon+\delta) \int(1+\delta \tau)^{\xi}\left\|\sigma^{-\frac{\nu}{2}}\left(\psi_{x x}, \bar{\psi}_{x x}\right)\right\|^{2} d \tau \\
& +\delta \xi \int_{0}^{t}(1+\delta \tau)^{\xi-1}\left\|\sigma^{-\frac{\nu}{2}}\left(\phi, \psi, \phi_{x}, \bar{\phi}, \bar{\psi}, \bar{\phi}_{x}\right)\right\|^{2} d \tau,
\end{aligned}
$$

with $\xi \geq 0$.

Proof. Adding (3.24)-(3.27) together, and multiplying the resulted equation by $\sigma^{-\nu}$ with the weight function $\sigma \geq 0$ satisfying (1.14) and (2.28), we integrate the resulted equation in $x$ over $\mathbb{R}_{+}$to obtain

$$
\begin{aligned}
& \frac{d}{d t} \int \sigma^{-\nu}\left(\mu \frac{\phi_{x}^{2}}{2}+\frac{\bar{\phi}_{x}^{2}}{2}+\widetilde{\rho}^{2} \phi_{x} \psi+\widetilde{n} \bar{\phi}_{x} \bar{\psi}\right) d x-\left[\sigma^{-\nu}\left(\mu u \frac{\phi_{x}^{2}}{2}+v \frac{\bar{\phi}_{x}^{2}}{2}-\widetilde{\rho}^{2} \phi_{t} \psi-\widetilde{n} \bar{\phi}_{t} \bar{\psi}\right)\right](t, 0) \\
& -a \nu \int \sigma^{-(\nu-1)}\left[\mu u \frac{\phi_{x}^{2}}{2}+v \frac{\bar{\phi}_{x}^{2}}{2}-\widetilde{\rho}^{2} \phi_{t} \psi-\widetilde{n} \bar{\phi}_{t} \bar{\psi}\right] d x+\int \sigma^{-\nu}\left(\widetilde{\rho}^{2} \frac{p_{1}^{\prime}(\rho)}{\rho} \phi_{x}^{2}+\widetilde{n} \frac{p_{2}^{\prime}(n)}{n} \bar{\phi}_{x}^{2}\right) d x \\
= & \sum_{i=1}^{6} \mathcal{J}_{i},
\end{aligned}
$$

where

$$
\begin{aligned}
\mathcal{J}_{1}= & -\int \sigma^{-\nu}\left[\widetilde{\rho}^{2}\left(\phi_{t}+u \phi_{x}\right) \psi_{x}+\widetilde{n}\left(\bar{\phi}_{t}+v \bar{\phi}_{x}\right) \bar{\psi}_{x}+2 \widetilde{\rho} \widetilde{\rho}_{x} \phi_{t} \psi+\widetilde{n}_{x} \bar{\phi}_{t} \bar{\psi}\right] d x, \\
\mathcal{J}_{2}= & \int \sigma^{-\nu}\left[-\mu \phi \phi_{x} \psi_{x x}-\bar{\phi}_{\bar{\phi}_{x}} \bar{\psi}_{x x}+\mu \widetilde{\rho}^{2}\left(\frac{1}{\rho}-\frac{1}{\widetilde{\rho}}\right) \phi_{x} \psi_{x x}+\widetilde{n}\left(\frac{1}{n}-\frac{1}{\widetilde{n}}\right)\left(\widetilde{n} \bar{\psi}_{x}\right)_{x} \bar{\phi}_{x}\right] d x, \\
\mathcal{J}_{3}= & \int \sigma^{-\nu}\left[\widetilde{\rho}^{2} \frac{n}{\rho}(\bar{\psi}-\psi) \phi_{x}-\widetilde{n}(\bar{\psi}-\psi) \bar{\phi}_{x}\right] d x, \\
\mathcal{J}_{4}= & -\int \sigma^{-\nu}\left(\frac{3}{2} \mu \psi_{x} \frac{\phi_{x}^{2}}{2}+\frac{3}{2} \bar{\psi}_{x} \bar{\phi}_{x}^{2}\right) d x+\int \sigma^{-\nu} \widetilde{n} \frac{\left(\bar{\phi} \bar{\psi}_{x}\right)_{x}}{n} \bar{\phi}_{x} d x, \quad \mathcal{J}_{5}=\int \sigma^{-\nu}\left(F_{1} \widetilde{\rho}^{2} \phi_{x}+F_{2} \widetilde{n} \bar{\phi}_{x}\right) d x, \\
\mathcal{J}_{6}= & -\int \sigma^{-\nu}\left[\mu\left(\frac{1}{2} \phi_{x} \widetilde{u}_{x}+\psi_{x} \widetilde{\rho}_{x}\right) \phi_{x}+\mu\left(\phi \widetilde{u}_{x}+\psi \widetilde{\rho}_{x}\right)_{x} \phi_{x}\right. \\
& \left.-\widetilde{n}_{x} \bar{\phi}_{x} \bar{\psi}_{x x}+\left(\frac{1}{2} \bar{\phi}_{x} \widetilde{v}_{x}+\bar{\psi}_{x} \widetilde{n}_{x}\right) \bar{\phi}_{x}-\left(\bar{\phi} \widetilde{v}_{x}+\bar{\psi} \widetilde{n}_{x}\right)_{x} \bar{\phi}_{x}\right] d x .
\end{aligned}
$$

Owing to (1.12), (3.7), and (4.23), we obtain the estimates for terms on the left hand side as below

$$
\begin{aligned}
- & {\left[\sigma^{-\nu}\left(\mu u \frac{\phi_{x}^{2}}{2}+v \frac{\bar{\phi}_{x}^{2}}{2}-\widetilde{\rho} \phi_{x} \psi-\widetilde{n} \bar{\phi}_{x} \bar{\psi}\right)\right](t, 0) \geq \frac{c}{\delta^{\nu}}\left(\phi_{x}^{2}(t, 0)+\bar{\phi}_{x}^{2}(t, 0)\right) \geq 0, } \\
& -a \nu \int \sigma^{-(\nu-1)}\left(\mu u \frac{\phi_{x}^{2}}{2}+v \frac{\bar{\phi}_{x}^{2}}{2}\right) d x \\
\geq & \frac{a \nu\left|u_{+}\right|}{2} \int \sigma^{-(\nu-1)}\left(\mu \phi_{x}^{2}+\bar{\phi}_{x}^{2}\right) d x-C(\varepsilon+\delta)\left\|\sigma^{-\frac{\nu-1}{2}}\left(\phi_{x}, \bar{\phi}_{x}\right)\right\|^{2}, \\
& -a \nu \int \sigma^{-(\nu-1)}\left(-\widetilde{\rho}^{2} \phi_{t} \psi-\widetilde{n} \bar{\phi}_{t} \bar{\psi}\right) d x \\
\geq & -C \varepsilon\left\|\sigma^{-\frac{\nu}{2}}\left(\phi_{x}, \bar{\phi}_{x}\right)\right\|^{2}-C\left\|\sigma^{-\frac{\nu-2}{2}}(\phi, \psi, \bar{\phi}, \bar{\psi})\right\|^{2}-C\left\|\sigma^{-\frac{\nu}{2}}\left(\psi_{x}, \bar{\psi}_{x}\right)\right\|^{2},
\end{aligned}
$$




$$
\int \sigma^{-\nu}\left(\widetilde{\rho}^{2} \frac{p_{1}^{\prime}(\rho)}{\rho} \phi_{x}^{2}+\widetilde{n} \frac{p_{2}^{\prime}(n)}{n} \bar{\phi}_{x}^{2}\right) d x \geq \frac{A_{1} \gamma \rho_{+}^{\gamma}}{2}\left\|\sigma^{-\frac{\nu}{2}} \phi_{x}\right\|^{2}+\frac{A_{2} \alpha n_{+}^{\alpha-1}}{2}\left\|\sigma^{-\frac{\nu}{2}} \bar{\phi}_{x}\right\|^{2}
$$

where we take $\delta$ and $\varepsilon$ small enough.

We turn to estimate terms on the right hand side of (4.46). With the help of (1.12), (4.23), Young inequality and Cauchy-Schwarz inequality, we gain

$$
\begin{aligned}
\left|\mathcal{J}_{1}\right| & \leq C \delta\left\|\sigma^{-\frac{\nu}{2}}\left(\phi_{x}, \bar{\phi}_{x}\right)\right\|^{2}+C\left\|\sigma^{-\frac{\nu}{2}}\left(\psi_{x}, \bar{\psi}_{x}\right)\right\|^{2}+C\left\|\sigma^{-\frac{\nu-2}{2}}(\phi, \psi, \bar{\phi}, \bar{\psi})\right\|^{2}, \\
\left|\mathcal{J}_{2}\right| & \leq C\left(\|(\phi, \bar{\phi})\| L^{\infty}+\delta\right)\left\|\sigma^{-\frac{\nu}{2}}\left(\phi_{x}, \bar{\phi}_{x}, \psi_{x x}, \bar{\psi}_{x x}\right)\right\|^{2}+C \delta^{2}\left\|\sigma^{-\frac{\nu}{2}} \bar{\psi}_{x}\right\|^{2} \\
& \leq C(\delta+\varepsilon)\left\|\sigma^{-\frac{\nu}{2}}\left(\phi_{x}, \bar{\phi}_{x}\right)\right\|^{2}+C(\delta+\varepsilon)\left\|\sigma^{-\frac{\nu}{2}}\left(\psi_{x x}, \bar{\psi}_{x x}\right)\right\|^{2}+C \delta\left\|\sigma^{-\frac{\nu}{2}} \bar{\psi}_{x}\right\|^{2}, \\
\left|\mathcal{J}_{3}\right| & \leq \frac{A_{1} \gamma \rho_{+}^{\gamma}}{8}\left\|\sigma^{-\frac{\nu}{2}} \phi_{x}\right\|^{2}+\frac{A_{2} \alpha n_{+}^{\alpha-1}}{8}\left\|\sigma^{-\frac{\nu}{2}} \bar{\phi}_{x}\right\|^{2}+C\left\|\sigma^{-\frac{\nu}{2}}(\bar{\psi}-\psi)\right\|^{2}, \\
\left|\mathcal{J}_{4}\right| & \leq C \varepsilon\left\|\sigma^{-\frac{\nu}{2}}\left(\phi_{x}, \bar{\phi}_{x}\right)\right\|^{2}+C \varepsilon\left\|\sigma^{-\frac{\nu}{2}}\left(\psi_{x x}, \bar{\psi}_{x x}\right)\right\|^{2}+C \varepsilon\left\|\sigma^{-\frac{\nu}{2}}\left(\psi_{x}, \bar{\psi}_{x}\right)\right\|^{2}, \\
\left|\mathcal{J}_{5}\right| & \leq C \delta\left\|\sigma^{-\frac{\nu}{2}}\left(\phi_{x}, \bar{\phi}_{x}\right)\right\|^{2}+C \delta\left\|\sigma^{-\frac{\nu-2}{2}}(\phi, \psi, \bar{\phi}, \bar{\psi})\right\|^{2}, \\
\left|\mathcal{J}_{6}\right| & \leq C \delta\left\|\sigma^{-\frac{\nu}{2}}\left(\phi_{x}, \bar{\phi}_{x}\right)\right\|^{2}+C \delta^{2}\left\|\sigma^{-\frac{\nu}{2}} \bar{\psi}_{x x}\right\|^{2}+C \delta\left\|\sigma^{-\frac{\nu}{2}}\left(\psi_{x}, \bar{\psi}_{x}\right)\right\|^{2}+C \delta\left\|\sigma^{-\frac{\nu-2}{2}}(\phi, \psi, \bar{\phi}, \bar{\psi})\right\|^{2} .
\end{aligned}
$$

Finally, the substitution of (4.47)-(4.56) into (4.46) for $\delta$ and $\varepsilon$ small enough leads to that

$$
\begin{aligned}
& \frac{d}{d t} \int \sigma^{-\nu}\left[\mu \frac{\phi_{x}^{2}}{2}+\frac{\bar{\phi}_{x}^{2}}{2}+\widetilde{\rho}^{2} \phi_{x} \psi+\widetilde{n} \bar{\phi}_{x} \bar{\psi}\right] d x+c\left\|\sigma^{-\frac{\nu-1}{2}}\left(\phi_{x}, \bar{\phi}_{x}\right)\right\|^{2} \\
& +\frac{A_{1} \gamma \rho_{+}^{\gamma}}{4}\left\|\sigma^{-\frac{\nu}{2}} \phi_{x}\right\|^{2}+\frac{A_{2} \alpha n_{+}^{\alpha-1}}{4}\left\|\sigma^{-\frac{\nu}{2}} \bar{\phi}_{x}\right\|^{2} \\
\leq & C(\varepsilon+\delta)\left\|\sigma^{-\frac{\nu}{2}}\left(\psi_{x x}, \bar{\psi}_{x x}\right)\right\|^{2}+C\left\|\sigma^{-\frac{\nu-2}{2}}(\phi, \psi, \bar{\phi}, \bar{\psi})\right\|^{2}+C\left\|\sigma^{-\frac{\nu}{2}}\left(\psi_{x}, \bar{\psi}_{x}, \bar{\psi}-\psi\right)\right\|^{2} .
\end{aligned}
$$

Multiplying (4.57) by $(1+\delta \tau)^{\xi}$ and integrating the resulted equation in $\tau$ over $[0, t]$, and using CauchySchwarz inequality and Lemma 4.8, we obtain the desired estimate (4.45). The proof of Lemma 4.9 is completed.

Lemma 4.10. Under the same conditions in Proposition 4.5 , then the solution $(\phi, \psi, \bar{\phi}, \bar{\psi})$ to the IBVP (3.3)-(3.7) satisfies for $t \in[0, T]$ that

$$
\begin{aligned}
& (1+\delta t)^{\xi}\left\|\sigma^{-\frac{\nu}{2}}\left(\psi_{x}, \bar{\psi}_{x}\right)\right\|^{2}+\int_{0}^{t}(1+\delta \tau)^{\xi}\left\|\sigma^{-\frac{\nu}{2}}\left(\psi_{x x}, \bar{\psi}_{x x}\right)\right\|^{2} d \tau \\
\leq & C\left\|\sigma^{-\frac{\lambda}{2}}\left(\phi_{0}, \psi_{0}, \phi_{0 x}, \psi_{0 x}, \bar{\phi}_{0}, \bar{\psi}_{0}, \bar{\phi}_{0 x}, \bar{\psi}_{0 x}\right)\right\|^{2} \\
& +C \delta \xi \int_{0}^{t}(1+\delta \tau)^{\xi-1}\left\|\sigma^{-\frac{\nu}{2}}\left(\phi, \psi, \phi_{x}, \psi_{x}, \bar{\phi}, \bar{\psi}, \bar{\phi}_{x}, \bar{\psi}_{x}\right)\right\|^{2} d \tau,
\end{aligned}
$$

with $\xi \geq 0$.

Proof. Multiplying $(3.3)_{2}$ by $-\sigma^{-\nu} \psi_{x x},(3.3)_{4}$ by $-\sigma^{-\nu} \bar{\psi}_{x x}$ respectively with the function $\sigma$ satisfying (1.14) and (2.28), then adding them together and integrating the resulted equation in $x$ over $\mathbb{R}_{+}$lead to

$$
\frac{d}{d t} \int \sigma^{-\nu}\left(\frac{\psi_{x}^{2}}{2}+\frac{\bar{\psi}_{x}^{2}}{2}\right) d x-a \nu \int \sigma^{-(\nu-1)}\left(\psi_{t} \psi_{x}+\bar{\psi}_{t} \bar{\psi}_{x}\right) d x+\int \sigma^{-\nu}\left(\frac{\mu}{\rho} \psi_{x x}^{2}+\bar{\psi}_{x x}^{2}\right) d x=\sum_{i=1}^{3} \mathcal{K}_{i},
$$


where

$$
\begin{aligned}
\mathcal{K}_{1}= & \int \sigma^{-\nu}\left[u \psi_{x} \psi_{x x}-\frac{n}{\rho}(\bar{\psi}-\psi) \psi_{x x}+\frac{p_{1}^{\prime}(\rho)}{\rho} \phi_{x} \psi_{x x}+v \bar{\psi}_{x} \bar{\psi}_{x x}+(\bar{\psi}-\psi) \bar{\psi}_{x x}+\frac{p_{2}{ }^{\prime}(n)}{n} \bar{\phi}_{x} \bar{\psi}_{x x}\right] d x, \\
\mathcal{K}_{2}= & \int \sigma^{-\nu}\left[\widetilde{u}_{x} \psi \psi_{x x}-\mu \widetilde{u}_{x x}\left(\frac{1}{\rho}-\frac{1}{\widetilde{\rho}}\right) \psi_{x x}+\left(\frac{p_{1}^{\prime}(\rho)}{\rho}-\frac{p_{1}^{\prime}(\widetilde{\rho})}{\widetilde{\rho}}\right) \psi_{x x} \widetilde{\rho}_{x}-\left(\frac{n}{\rho}-\frac{\widetilde{n}}{\widetilde{\rho}}\right)(\widetilde{v}-\widetilde{u}) \psi_{x x}+\widetilde{v}_{x} \bar{\psi} \bar{\psi}_{x x}\right. \\
& \left.+\left(\widetilde{n} \widetilde{v}_{x}\right)_{x}\left(\frac{1}{n}-\frac{1}{\widetilde{n}}\right) \bar{\psi}_{x x}+\left(\frac{p_{2}^{\prime}(n)}{n}-\frac{p_{2}^{\prime}(\widetilde{n})}{\widetilde{n}}\right) \bar{\psi}_{x x} \widetilde{n}_{x}-\frac{\widetilde{n}_{x}}{\widetilde{n}} \bar{\psi}_{x} \bar{\psi}_{x x}-\frac{\left(\bar{\phi} \widetilde{v}_{x}\right)_{x}}{n} \bar{\psi}_{x x}\right] d x, \\
\mathcal{K}_{3}= & -\int \sigma^{-\nu}\left[\frac{\left(\bar{\phi} \bar{\psi}_{x}\right)_{x}}{n} \bar{\psi}_{x x}+\left(\frac{1}{n}-\frac{1}{\widetilde{n}}\right)\left(\widetilde{n} \bar{\psi}_{x}\right)_{x} \bar{\psi}_{x x}\right] d x .
\end{aligned}
$$

First, we estimate terms in the left side of (4.59). The second term is estimated as follows:

$$
\begin{aligned}
& a \nu \int \sigma^{-(\nu-1)}\left(\psi_{t} \psi_{x}+\bar{\psi}_{t} \bar{\psi}_{x}\right) d x \\
\leq & C \delta\left\|\sigma^{-\frac{\nu}{2}}\left(\psi_{x x}, \bar{\psi}_{x x}\right)\right\|^{2}+C \delta\left\|\sigma^{-\frac{\nu}{2}}\left(\phi_{x}, \psi_{x}, \bar{\phi}_{x}, \bar{\psi}_{x}, \bar{\psi}-\psi\right)\right\|^{2}+C \delta\left\|\sigma^{-\frac{\nu-2}{2}}(\phi, \psi, \bar{\phi}, \bar{\psi})\right\|^{2},
\end{aligned}
$$

where we have used $(1.12),(3.3)_{2},(3.3)_{4},(4.23)$ and Cauchy-Schwarz inequality.

With the help of $\delta$ and $\varepsilon$ small enough, the third term is estimated as follows:

$$
\begin{aligned}
\int \sigma^{-\nu}\left(\frac{\mu}{\rho} \psi_{x x}^{2}+\bar{\psi}_{x x}^{2}\right) d x & \geq\left[\frac{\mu}{\rho_{+}}-C(\varepsilon+\delta)\right]\left\|\sigma^{-\frac{\nu}{2}} \psi_{x x}\right\|^{2}+\left\|\sigma^{-\frac{\nu}{2}} \bar{\psi}_{x x}\right\|^{2} \\
& \geq \frac{\mu}{2 \rho_{+}}\left\|\sigma^{-\frac{\nu}{2}} \psi_{x x}\right\|^{2}+\left\|\sigma^{-\frac{\nu}{2}} \bar{\psi}_{x x}\right\|^{2} .
\end{aligned}
$$

We turn to estimate terms on the right hand side of (4.59). With the help of (1.12), (4.23) and CauchySchwarz inequality, we obtain

$$
\begin{aligned}
\left|\mathcal{K}_{1}\right| & \leq \frac{\mu}{8 \rho_{+}}\left\|\sigma^{-\frac{\nu}{2}} \psi_{x x}\right\|^{2}+\frac{1}{8}\left\|\sigma^{-\frac{\nu}{2}} \bar{\psi}_{x x}\right\|^{2}+C\left\|\sigma^{-\frac{\nu}{2}}\left(\phi_{x}, \psi_{x}, \bar{\phi}_{x}, \bar{\psi}_{x}, \bar{\psi}-\psi\right)\right\|^{2}, \\
\left|\mathcal{K}_{2}\right| & \leq C \delta\left\|\sigma^{-\frac{\nu}{2}}\left(\psi_{x x}, \bar{\psi}_{x x}\right)\right\|^{2}+C \delta\left\|\sigma^{-\frac{\nu-2}{2}}(\phi, \psi, \bar{\phi}, \bar{\psi})\right\|^{2}+C \delta\left\|\sigma^{-\frac{\nu}{2}}\left(\bar{\phi}_{x}, \bar{\psi}_{x}\right)\right\|^{2}, \\
\left|\mathcal{K}_{3}\right| & \leq C(\varepsilon+\delta)\left\|\sigma^{-\frac{\nu}{2}}\left(\bar{\psi}_{x}, \bar{\psi}_{x x}\right)\right\|^{2} .
\end{aligned}
$$

Finally, we substitute (4.60)-(4.64) into (4.59) to gain under the condition $\delta$ and $\varepsilon$ small enough that

$$
\begin{aligned}
& \frac{d}{d t} \int \sigma^{-\nu}\left(\frac{\psi_{x}^{2}}{2}+\frac{\bar{\psi}_{x}^{2}}{2}\right) d x+\frac{\mu}{4 \rho_{+}}\left\|\sigma^{-\frac{\nu}{2}} \psi_{x x}\right\|^{2}+\frac{1}{4}\left\|\sigma^{-\frac{\nu}{2}} \bar{\psi}_{x x}\right\|^{2} \\
\leq & C\left\|\sigma^{-\frac{\nu}{2}}\left(\phi_{x}, \psi_{x}, \bar{\phi}_{x}, \bar{\psi}_{x}, \bar{\psi}-\psi\right)\right\|^{2}+C \delta\left\|\sigma^{-\frac{\nu-2}{2}}(\phi, \psi, \bar{\phi}, \bar{\psi})\right\|^{2}
\end{aligned}
$$

Multiplying (4.65) by $(1+\delta \tau)^{\xi}$ and integrating the resulted inequality in $\tau$ over $[0, t]$, and using Lemmas 4.8-4.9 and the smallness of $\delta$ and $\varepsilon$, we obtain (4.58). The proof of Lemma 4.10 is completed.

Proof of Proposition 4.5 With the help of Lemmas 4.8-4.10, it holds for $\delta$ and $\varepsilon$ suitably small that

$$
\begin{aligned}
& (1+\delta t)^{\xi}\left\|\sigma^{-\frac{\nu}{2}}(\phi, \psi, \bar{\phi}, \bar{\psi})\right\|_{1}^{2}+\int_{0}^{t}(1+\delta \tau)^{\xi}\left\|\sigma^{-\frac{\nu-2}{2}}(\phi, \psi, \bar{\phi}, \bar{\psi})\right\|^{2} d \tau+\int_{0}^{t}(1+\delta \tau)^{\xi}\left\|\sigma^{-\frac{\nu}{2}}\left(\phi_{x}, \psi_{x}, \bar{\phi}_{x}, \bar{\psi}_{x}\right)\right\|^{2} \\
& +C \int_{0}^{t}(1+\delta \tau)^{\xi}\left\|\sigma^{-\frac{\nu}{2}}\left(\psi_{x x}, \bar{\psi}_{x x}, \bar{\psi}-\psi\right)\right\|^{2}+C \frac{1}{\delta^{\nu}} \int_{0}^{t}(1+\delta \tau)^{\xi}\left(\phi^{2}(t, 0)+\bar{\phi}^{2}(t, 0)\right) d \tau \\
\leq & C\left\|\sigma^{-\frac{\lambda}{2}}\left(\phi_{0}, \psi_{0}, \bar{\phi}_{0}, \bar{\psi}_{0}\right)\right\|_{1}^{2}+C \delta \xi \int_{0}^{t}(1+\delta \tau)^{\xi-1}\left\|\sigma^{-\frac{\nu}{2}}(\phi, \psi, \bar{\phi}, \bar{\psi})\right\|_{1}^{2} d \tau
\end{aligned}
$$


where $C>0$ is a positive constant independent of $T, \nu$ and $\xi$. Applying similar induction arguments as in $[3,14,25]$ to $(4.66)$, we have

$$
\begin{aligned}
& (1+\delta t)^{\frac{\lambda-\nu}{2}+\beta}\left\|\sigma^{-\frac{\nu}{2}}\left(\phi, \psi, \phi_{x}, \psi_{x}, \bar{\phi}, \bar{\psi}, \bar{\phi}_{x}, \bar{\psi}_{x}\right)\right\|^{2}+\int_{0}^{t}(1+\delta \tau)^{\frac{\lambda-\nu}{2}+\beta}\left\|\sigma^{-\frac{\nu-2}{2}}(\phi, \bar{\phi}, \psi, \bar{\psi})\right\|^{2} \\
& +\int_{0}^{t}(1+\delta \tau)^{\frac{\lambda-\nu}{2}+\beta}\left\|\sigma^{-\frac{\nu}{2}}\left(\phi_{x}, \psi_{x}, \psi_{x x}, \bar{\phi}_{x}, \bar{\psi}_{x}, \bar{\psi}_{x x}, \bar{\psi}-\psi\right)\right\|^{2} \\
\leq & C(1+\delta t)^{\beta}\left\|\sigma^{-\frac{\lambda}{2}}\left(\phi_{0}, \psi_{0}, \phi_{0 x}, \psi_{0 x}, \bar{\phi}_{0}, \bar{\psi}_{0}, \bar{\phi}_{0 x}, \bar{\psi}_{0 x}\right)\right\|^{2}
\end{aligned}
$$

for $\beta>0$, which implies

$$
\left\|\sigma^{-\frac{\nu}{2}}\left(\phi, \psi, \phi_{x}, \psi_{x}, \bar{\phi}, \bar{\psi}, \bar{\phi}_{x}, \bar{\psi}_{x}\right)(t)\right\|^{2} \leq C(1+\delta t)^{-\frac{\lambda-\nu}{4}}\left\|\sigma^{-\frac{\lambda}{2}}\left(\phi_{0}, \psi_{0}, \phi_{0 x}, \psi_{0 x}, \bar{\phi}_{0}, \bar{\psi}_{0}, \bar{\phi}_{0 x}, \bar{\psi}_{0 x}\right)\right\|^{2}
$$

\section{Acknowledgments}

The research of the paper is supported by the National Natural Science Foundation of China (Nos. 11931010, 11871047, 11671384), by the key research project of Academy for Multidisciplinary Studies, Capital Normal University, and by the Capacity Building for Sci-Tech Innovation-Fundamental Scientific Research Funds (No. 007/20530290068).

\section{References}

[1] F. Bubba, B. Perthame, C. Pouchol, M. Schmidtchen, Hele-Shaw limit for a system of two reaction-(cross)diffusion equations for living tissues. Arch. Ration. Mech. Anal. 236 (2020), no. 2, 735-766.

[2] J. Carr, Applications of Center Manifold Theory, Springer-Verlag, 1981.

[3] Y.Z. Chen, H. Hong, X,D. Shi, Convergence rate of stationary solutions to outflow problem for full NavierStokes equations. Appl. Anal. 98 (2019), no. 7, 1267-1288.

[4] R.J. Duan, X.F. Yang, Stability of rarefaction wave and boundary layer for outflow problem on the two-fluid Navier- Stokes-Poisson equations, Commun. Pure Appl. Anal. 12 (2013) 985-1014.

[5] L.L. Fan, H.X. Liu, T. Wang, H.J. Zhao, Inflow problem for the one-dimensional compressible Navier-Stokes equations under large initial perturbation. J. Differential Equations 257 (2014), no. 10, 3521-3553.

[6] C.C. Hao and H.L. Li, Well-posedness for a multidimensional viscous liquid-gas two-phase flow model, SIAM J. Math. Anal. 44 (2012), 1304-1332.

[7] H. Hong, X.D. Shi, T. Wang, Stability of stationary solutions to the inflow problem for the two-fluid nonisentropic Navier-Stokes-Poisson system. J. Differential Equations 265 (2018), no. 4, 1129-1155.

[8] H. Hong, T. Wang, Stability of stationary solutions to the inflow problem for full compressible Navier-Stokes equations with a large initial perturbation. SIAM J. Math. Anal. 49 (2017), no. 3, 2138-2166.

[9] F.M. Huang, A. Matsumura, X.D. Shi, Viscous shock wave and boundary layer solution to an inflow problem for compressible viscous gas. Comm. Math. Phys. 239 (2003), no. 1-2, 261-285. 
[10] F.M. Huang, J. Li, X.D. Shi, Asymptotic behavior of solutions to the full compressible Navier-Stokes equations in the half space. Commun. Math. Sci. 8 (2010), no. 3, 639-654.

[11] F.M. Huang, X.H. Qin, Stability of boundary layer and rarefaction wave to an outflow problem for compressible Navier-Stokes equations under large perturbation. J. Differential Equations 246 (2009), no. 10, 4077-4096.

[12] M. Ishii and T. Hibiki, Thermo-fluid Dynamics of Two-Phase Flow. New York: Springer-Verlag, 2006.

[13] S. Kawashima, K. Kurata, Hardy type inequality and application to the stability of degenerate stationary waves. J. Funct. Anal. 257 (2009), no. 1, 1-19.

[14] S. Kawashima, A. Matsumura, Asymptotic stability of traveling wave solutions of systems for one-dimensional gas motion, Comm. Math. Phys. 101 (1985), no. 1, 97-127.

[15] S. Kawashima, T. Nakamura, S. Nishibata, P.C. Zhu, Stationary waves to viscous heat-conductive gases in half-space: existence, stability and convergence rate. Math. Models Methods Appl. Sci. 20 (2010), no. 12, 2201-2235.

[16] S. Kawashima, S. Nishibata and P. Zhu, Asymptotic stability of the stationary solution to the compressible Navier-Stokes equations in the half space, Comm. Math. Phys. 240 (2003), 483-500.

[17] S. Kawashima, P.C. Zhu, Asymptotic stability of nonlinear wave for the compressible Navier-Stokes equations in the half space. J. Differential Equations 244 (2008), no. 12, 3151-3179.

[18] S. Kawashima, P.C. Zhu, Asymptotic stability of rarefaction wave for the Navier-Stokes equations for a compressible fluid in the half space. Arch. Ration. Mech. Anal. 194 (2009), no. 1, 105-132.

[19] H.L. Li, T. Wang, Y. Wang, Wave phenomena to the three-dimensional fluid-particle model, preprint, 2020.

[20] H.L. Li, S. Zhao, Existence and nonlinear stability of stationary solutions to the full two-phase flow model in a half line. Appl. Math. Lett. 116 (2021), 107039.

[21] T. Lorenzi, A. Lorz, B. Perthame, On interfaces between cell populations with different mobilities. Kinet. Relat. Models 10 (2017), no. 1, 299-311.

[22] A. Matsumura, Inflow and outflow problems in the half space for a one-dimensional isentropic model system of compressible viscous gas, Methods Appl. Anal. 8(2001), 645-666.

[23] A. Matsumura, K. Nishihara, Large-time behaviors of solutions to an inflow problem in the half space for a one-dimensional system of compressible viscous gas. Comm. Math. Phys. 222 (2001), no. 3, 449-474.

[24] A. Mellet, A. Vasseur, Asymptotic analysis for a Vlasov-Fokker-Planck/compressible Navier- Stokes system of equations, Comm. Math. Phys. 281 (2008), no. 3, 573-596.

[25] M. Nishikawa, Convergence rate to the traveling wave for viscous conservation laws, Funkcial. Ekvac. 41 (1998), no. 1, 107-132.

[26] T. Nakamura, S. Nishibata, Stationary wave associated with an inflow problem in the half line for viscous heat-conductive gas. J. Hyperbolic Differ. Equ. 8 (2011), no. 4, 651-670. 
[27] T. Nakamura, S. Nishibata, Existence and asymptotic stability of stationary waves for symmetric hyperbolicparabolic systems in half-line. Math. Models Methods Appl. Sci. 27 (2017), no. 11, 2071-2110.

[28] T. Nakamura, S. Nishibata, N. Usami, Convergence rate of solutions towards the stationary solutions to symmetric hyperbolic-parabolic systems in half space. Kinet. Relat. Models 11 (2018), no. 4, 757-793.

[29] T. Nakamura, S. Nishibata and T. Yuge, Convergence rate of solutions toward stationary solutions to the compressible Navier-Stokes equation in a half line, J. Differential Equations 241 (2007), 94-111.

[30] X.H. Qin, Large-time behaviour of solutions to the outflow problem of full compressible Navier-Stokes equations. Nonlinearity 24 (2011), no. 5, 1369-1394.

[31] X.H. Qin, Y. Wang, Stability of wave patterns to the inflow problem of full compressible Navier-Stokes equations. SIAM J. Math. Anal. 41 (2009), no. 5, 2057-2087.

[32] X.H. Qin, Y. Wang, Large-time behavior of solutions to the inflow problem of full compressible Navier-Stokes equations. SIAM J. Math. Anal. 43 (2011), no. 1, 341-366.

[33] L. Wan, T. Wang, H.J. Zhao, Asymptotic stability of wave patterns to compressible viscous and heatconducting gases in the half-space. J. Differential Equations 261 (2016), no. 11, 5949-5991.

[34] L. Wan, T. Wang, Q.Y. Zou, Stability of stationary solutions to the outflow problem for full compressible Navier-Stokes equations with large initial perturbation. Nonlinearity 29 (2016), no. 4, 1329-1354.

[35] H.Y. Yin, J.S. Zhang, C.J. Zhu, Stability of the superposition of boundary layer and rarefaction wave for outflow problem on the two-fluid Navier-Stokes-Poisson system, Nonlinear Anal. Real World Appl. 31 (2016) 492-512.

[36] H.Y. Yin, Converge rates towards stationary solutions for the outflow problem of planar magnetohydrodynamics on a half line. Proc. Roy. Soc. Edinburgh Sect. A 149 (2019), no. 5, 1291-1322.

[37] H.Y. Yin, C.J. Zhu, Convergence rate of solutions toward stationary solutions to a viscous liquid-gas twophase flow model in a half line, Commun. Pure Appl. Anal. 14 (2015), no. 5, 2021-2042.

[38] F. Zhou, Y.P. Li, Convergence rate of solutions toward stationary solutions to the bipolar Navier-StokesPoisson equations in a half line, Bound. Value Probl. 2013 (2013) 1. 


\title{
Existence and Nonlinear Stability of Steady-States to Outflow Problem for the Full Two-Phase Flow
}

\author{
Hai-Liang Li *a,b and Shuang Zhao ${ }^{\dagger a, b}$ \\ ${ }^{a}$ School of Mathematical Sciences, Capital Normal University, Beijing 100048, P.R. China. \\ ${ }^{\mathrm{b}}$ Academy for Multidisciplinary Studies, Capital Normal University, Beijing 100048, P.R. China.
}

\begin{abstract}
The outflow problem for the viscous full two-phase flow model in a half line is investigated in the present paper. The existence, uniqueness and the nonlinear time stability of the steady-state are shown corresponding to supersonic, sonic or subsonic flow at far field. Furthermore, we can obtain either the exponential time decay rate for supersonic state or the algebraic time decay rate for supersonic state and sonic state in the weighted Sobolev space.
\end{abstract}

Key words. Full two-phase flow, outflow problem, stationary solution, nonlinear stability.

\section{Introduction}

Two-phase flow models play important roles in applied scientific areas, for instance, nuclear, engines, chemical engineering, medicine, oil-gas, fluidization, waste water treatment, liquid crystals, lubrication, biomedical flows $[8,13,22]$, etc. In this paper, we consider the full two-phase flow model which can be formally obtained from a Vlasov-Fokker-Planck equation coupled with the compressible Navier-Stokes equations through the Chapman-Enskog expansion [20].

We are concerned with the initial-boundary value problem (IBVP) for the full two-phase flow model as follows:

$$
\left\{\begin{array}{l}
\rho_{t}+(\rho u)_{x}=0 \\
(\rho u)_{t}+\left[\rho u^{2}+p_{1}(\rho)\right]_{x}=\left(\mu u_{x}\right)_{x}+n(v-u), \\
n_{t}+(n v)_{x}=0 \\
(n v)_{t}+\left[n v^{2}+p_{2}(n)\right]_{x}=\left(n v_{x}\right)_{x}-n(v-u), \quad x>0, t>0
\end{array}\right.
$$

*E-mail: hailiang.li.math@gmail.com (H.-L. Li)

${ }^{\dagger}$ Corresponding author: E-mail: shuangzhaomath@163.com(S. Zhao) 
where $\rho>0$ and $n>0$ stand for the densities of two fluids, and $u, v$ are the velocities of two fluids. The constant $\mu>0$ is the viscosity coefficient and the pressure-density functions take the form

$$
p_{1}(\rho)=A_{1} \rho^{\gamma}, \quad p_{2}(n)=A_{2} n^{\alpha}
$$

with $A_{1}>0, A_{2}>0, \gamma \geq 1$ and $\alpha \geq 1$. The initial data are given by

$$
\begin{gathered}
(\rho, u, n, v)(0, x)=\left(\rho_{0}, u_{0}, n_{0}, v_{0}\right)(x), \quad \inf _{x \in \mathbb{R}_{+}} \rho_{0}(x)>0, \quad \inf _{x \in \mathbb{R}_{+}} n_{0}(x)>0, \\
\lim _{x \rightarrow+\infty}\left(\rho_{0}, u_{0}, n_{0}, v_{0}\right)(x)=\left(\rho_{+}, u_{+}, n_{+}, u_{+}\right), \quad \rho_{+}>0, \quad n_{+}>0,
\end{gathered}
$$

and the outflow boundary condition is imposed

$$
(u, v)(t, 0)=\left(u_{-}, u_{-}\right), \quad u_{-}<0,
$$

where $\rho_{+}>0, n_{+}>0, u_{+}$and $u_{-}<0$ are constants.

There are important progress made recently about the global existence and time decay rates of solutions for the two-phase flow models $[3,5-8,14,29,31,32,34-36,39]$. For instance, the global existence of three-dimensional strong solutions to Cauchy problem for Euler-NS model was proved in [3]. Later, time decay rates of the three-dimensional strong solution to Cauchy problem for Euler-NS model were obtained in [31]. The global existence and time decay rates of three-dimensional strong solutions to the initial boundary problem for Euler-NS model was proved in [14]. The global well-posedness of strong solutions to Cauchy problem in Besov space or Sobolev space was obtained in $[8,32,39]$ for drift-flux models. The existence of multi-dimensional global weak solutions for drift-flux models to both Cauchy problem [6,34] and the initial boundary value problem [29] was proved. The global weak solution to the free boundary value problem in $1 \mathrm{D}$ was shown in $[5,7,35,36]$ for drift-flux models.

There are important progress made recently about the existence and nonlinear stability of steadystates for the inflow/outflow problem of the compressible Navier-Stokes system $[2,4,9-12,15,18,19,21$, $23,25-28,30,33,38,40]$. Among them, the existence and nonlinear stability of steady-states to the inflow problem of Navier-Stokes equations in a half line was obtained in $[10,11,21,25,30]$ for both subsonic and sonic cases. Concerning with the outflow problem of Navier-Stokes equations, the nonlinear stability of the steady-states for both supersonic and sonic cases in a half line was proved in $[12,19,26]$, and the stability of the multidimensional planar stationary solution for both supersonic and sonic cases was obtained in [15]. The existence and time decay rates of the solution to the outflow problem for Navier-Stokes equations in a half line were shown in $[27,28]$ for supersonic and sonic cases. The existence and nonlinear stability of steady-states to the outflow problem for full compressible Navier-Stokes equations $[2,18]$ were shown for supersonic, sonic and subsonic cases, and the time decay rates for the supersonic case or sonic case were obtained. 
However, there are also important results about the outflow/inflow problem for two-phase flow models except for $[4,9,33,38,40]$. Among them, the existence of steady-states for supersonic and sonic cases, and the nonlinear stability of steady-states for the supersonic case to the outflow problem were gained in [38] concerning with the drift-flux model. For the two-fluid Navier-Stokes-Poisson system, the existence and nonlinear stability of steady-states to the outflow problem for supersonic and sonic cases in $[4,33,40]$ were proved. The existence and nonlinear stability of steady-states to inflow problem of the two-fluid Navier-Stokes-Poisson system for both subsonic and sonic cases were shown in [9].

However, there is no result about the nonlinear time stability and time decay rates of steady-states for the outflow problem (1.1)-(1.5). The main purpose of this paper is to prove the nonlinear time stability of steady-states for supersonic, sonic and subsonic cases, and obtain either the exponential time decay rate for the supersonic flow or the algebraic time decay rate for both supersonic and sonic flows.

The steady-state $(\widetilde{\rho}, \widetilde{u}, \widetilde{n}, \widetilde{v})(x)$ to the problem (1.1)-(1.5) satisfies the following system

$$
\left\{\begin{array}{l}
(\widetilde{\rho u})_{x}=0, \\
{\left[\widetilde{\rho} \widetilde{u}^{2}+p_{1}(\widetilde{\rho})\right]_{x}=\left(\mu \widetilde{u}_{x}\right)_{x}+\widetilde{n}(\widetilde{v}-\widetilde{u}),} \\
(\widetilde{n} \widetilde{v})_{x}=0, \\
{\left[\widetilde{n} \widetilde{v}^{2}+p_{2}(\widetilde{n})\right]_{x}=\left(\widetilde{n} \widetilde{v}_{x}\right)_{x}-\widetilde{n}(\widetilde{v}-\widetilde{u}), \quad x>0,}
\end{array}\right.
$$

for the boundary conditions and spatial far field conditions

$$
(\widetilde{u}, \widetilde{v})(0)=\left(u_{-}, u_{-}\right), \quad \lim _{x \rightarrow \infty}(\widetilde{\rho}, \widetilde{u}, \widetilde{n}, \widetilde{v})(x)=\left(\rho_{+}, u_{+}, n_{+}, u_{+}\right) . \quad \inf _{x \in \mathbb{R}_{+}} \widetilde{n}(x)>0, \quad \inf _{x \in \mathbb{R}_{+}} \widetilde{n}(x)>0
$$

Integrating $(1.6)_{1}$ and $(1.6)_{3}$ over $(x,+\infty)$ leads to

$$
\widetilde{u}=\frac{\rho_{+}}{\widetilde{\rho}} u_{+}, \quad \widetilde{v}=\frac{n_{+}}{\widetilde{n}} u_{+},
$$

which implies the following relationship

$$
u_{+}=\frac{\widetilde{n}(0)}{n_{+}} u_{-}=\frac{\widetilde{\rho}(0)}{\rho_{+}} u_{-}<0
$$

is necessary for the existence of the steady-state to BVP (1.6)-(1.7).

Define the generalized Mach number $M_{+}$and the generalized sound speed $c_{+}$as below

$$
M_{+}:=\frac{\left|u_{+}\right|}{c_{+}}, \quad c_{+}:=\left(\frac{A_{1} \gamma \rho_{+}^{\gamma}+A_{2} \alpha n_{+}^{\alpha}}{\rho_{+}+n_{+}}\right)^{\frac{1}{2}} .
$$

Then, we have the following results about the existence and uniqueness of the steady-state.

Theorem 1.1. Let $\delta:=\left|u_{-}-u_{+}\right|>0$ and $u_{+}<0$ hold. Then there exists a set $\Omega_{-} \subset \mathbb{R}_{-}$such that if $u_{-} \in \Omega_{-}$and $\delta$ sufficiently small, there exists a unique strong solution $(\widetilde{\rho}, \widetilde{u}, \widetilde{n}, \widetilde{v})(x)$ to the boundary value problem (1.6)-(1.7) which satisfies either for $M_{+} \neq 1$ that

$$
\left|\partial_{x}^{k}\left(\widetilde{\rho}-\rho_{+}, \widetilde{u}-u_{+}, \widetilde{n}-n_{+}, \widetilde{v}-u_{+}\right)\right| \leq C_{1} \delta e^{-c_{0} x}, \quad k=0,1,2,3,
$$


or for $M_{+}=1$ that

$$
\left|\partial_{x}^{k}\left(\widetilde{\rho}-\rho_{+}, \widetilde{u}-u_{+}, \widetilde{n}-n_{+}, \widetilde{v}-u_{+}\right)\right| \leq C_{2} \frac{\delta^{k+1}}{(1+\delta x)^{k+1}}, k=0,1,2,3,
$$

and

$$
\left(\widetilde{u}_{x}, \widetilde{v}_{x}\right)=\left(a \sigma^{2}(x), a \sigma^{2}(x)\right)+O\left(|\sigma(x)|^{3}\right),
$$

where $\sigma(x)$ is a smooth function satisfying $\sigma_{x}=-a \sigma^{2}+O\left(|\sigma|^{3}\right)$ and

$$
c_{1} \frac{\delta}{1+\delta x} \leq \sigma(x) \leq C_{3} \frac{\delta}{1+\delta x}, \quad\left|\partial_{x}^{k} \sigma(x)\right| \leq C_{3} \frac{\delta^{k+1}}{(1+\delta x)^{k+1}}, \quad k=0,1,2,3 \ldots,
$$

and $C_{i}>0, i=1,2,3, c_{0}>0 c_{1}>0$ and $a>0$ are positive constants.

Then, we have the nonlinear stability of the steady-state to the IBVP (1.1)-(1.5) for supersonic, sonic and subsonic cases.

Theorem 1.2. Let the same conditions in Theorem 1.1 hold. In addition, assume that $\left|p_{1}^{\prime}\left(\rho_{+}\right)-p_{2}^{\prime}\left(n_{+}\right)\right| \leq$ $\sqrt{2}\left|u_{+}\right| \min \left\{\left(1+\frac{\rho_{+}}{n_{+}}\right)\left[(\gamma-1) p_{1}^{\prime}\left(\rho_{+}\right)\right]^{\frac{1}{2}},\left(1+\frac{n_{+}}{\rho_{+}}\right)\left[(\alpha-1) p_{2}^{\prime}\left(n_{+}\right)\right]^{\frac{1}{2}}\right\}$ hold if $M_{+}=1$. Then, there exists a small positive constant $\varepsilon_{0}>0$ such that if

$$
\left\|\left(\rho_{0}-\widetilde{\rho}, u_{0}-\widetilde{u}, n_{0}-\widetilde{n}, v_{0}-\widetilde{v}\right)\right\|_{H^{1}}+\delta \leq \varepsilon_{0}
$$

the initial boundary value problem (1.1)-(1.5) has a unique global solution $(\rho, u, n, v)(t, x)$ satisfying

$$
\left\{\begin{array}{l}
(\rho-\widetilde{\rho}, u-\widetilde{u}, n-\widetilde{n}, v-\widetilde{v}) \in C\left([0,+\infty) ; H^{1}\right), \\
(\rho-\widetilde{\rho}, n-\widetilde{n})_{x} \in L^{2}\left([0,+\infty) ; L^{2}\right), \\
(u-\widetilde{u}, v-\widetilde{v})_{x} \in L^{2}\left([0,+\infty) ; H^{1}\right),
\end{array}\right.
$$

and

$$
\lim _{t \rightarrow+\infty} \sup _{x \in \mathbb{R}_{+}}|(\rho-\widetilde{\rho}, u-\widetilde{u}, n-\widetilde{n}, v-\widetilde{v})(t, x)|=0 .
$$

In addition, we have the time convergence rates of the steady-state to the IBVP (1.1)-(1.5) for both supersonic and sonic cases.

Theorem 1.3. Assume that the same conditions in Theorem 1.1 hold. Then, the following results hold. (i) For $M_{+}>1$ and $\lambda>0$, if the initial data satisfy $\left((1+x)^{\frac{\lambda}{2}}\left(\rho_{0}-\widetilde{\rho}\right),(1+x)^{\frac{\lambda}{2}}\left(u_{0}-\widetilde{u}\right),(1+x)^{\frac{\lambda}{2}}\left(n_{0}-\right.\right.$ $\left.\widetilde{n}),(1+x)^{\frac{\lambda}{2}}\left(v_{0}-\widetilde{v}\right)\right) \in L^{2}\left(\mathbb{R}_{+}\right)$and

$$
\left\|\left(\rho_{0}-\widetilde{\rho}, u_{0}-\widetilde{u}, n_{0}-\widetilde{n}, v_{0}-\widetilde{v}\right)\right\|_{H^{1}}+\delta \leq \varepsilon_{0},
$$

for a small positive constant $\varepsilon_{0}>0$, then the solution $(\rho, u, n, v)(t, x)$ to the IBVP (1.1)-(1.5) satisfies

$$
\|(\rho-\widetilde{\rho}, u-\widetilde{u}, n-\widetilde{n}, v-\widetilde{v})(t)\|_{L^{\infty}} \leq C_{4} \delta_{0}(1+t)^{-\frac{\lambda}{2}},
$$


where $C_{4}>0$ is a positive constant independent of time and $\delta_{0}:=\left\|\left(\rho_{0}-\widetilde{\rho}, u_{0}-\widetilde{u}, n_{0}-\widetilde{n}, v_{0}-\widetilde{v}\right)\right\|_{H^{1}}+$ $\left\|(1+x)^{\frac{\lambda}{2}}\left(\rho_{0}-\widetilde{\rho}, u_{0}-\widetilde{u}, n_{0}-\widetilde{n}, v_{0}-\widetilde{v}\right)\right\|_{L^{2}}$ is a non-negative constant.

(ii) For $M_{+}=1,1 \leq \lambda<2+\sqrt{8+\frac{1}{1+b^{2}}}$ and $b:=\frac{\rho_{+}\left(u_{+}^{2}-p_{1}^{\prime}\left(\rho_{+}\right)\right)}{\left|u_{+}\right| \sqrt{\left(\mu+n_{+}\right) n_{+}}}$, if for $\nu \in(0, \lambda]$, there exists a small positive constant $\varepsilon_{0}>0$ such that if

$$
\left\|\sigma^{-\frac{\lambda}{2}}\left(\rho_{0}-\widetilde{\rho}, u_{0}-\widetilde{u}, n_{0}-\widetilde{n}, v_{0}-\widetilde{v}\right)\right\|_{H^{1}}+\delta^{\frac{1}{2}} \leq \varepsilon_{0}
$$

then the IBVP (1.1)-(1.5) has a unique global solution $(\rho, u, n, v)(t, x)$ satisfying

$$
\left\{\begin{array}{l}
\left(\sigma^{-\frac{\nu}{2}}(\rho-\widetilde{\rho}), \sigma^{-\frac{\nu}{2}}(u-\widetilde{u}), \sigma^{-\frac{\nu}{2}}(n-\widetilde{n}), \sigma^{-\frac{\nu}{2}}(v-\widetilde{v})\right) \in C\left([0,+\infty) ; H^{1}\right), \\
\left(\sigma^{-\frac{\nu}{2}}(\rho-\widetilde{\rho})_{x}, \sigma^{-\frac{\nu}{2}}(n-\widetilde{n})_{x}\right) \in L^{2}\left([0,+\infty) ; L^{2}\right), \\
\left(\sigma^{-\frac{\nu}{2}}(u-\widetilde{u})_{x}, \sigma^{-\frac{\nu}{2}}(v-\widetilde{v})_{x}\right) \in L^{2}\left([0,+\infty) ; H^{1}\right)
\end{array}\right.
$$

and

$$
\left\|\sigma^{-\frac{\nu}{2}}(\rho-\widetilde{\rho}, u-\widetilde{u}, n-\widetilde{n}, v-\widetilde{v})(t)\right\|_{H^{1}} \leq C_{5} \delta_{1}(1+t)^{-\frac{\lambda-\nu}{4}},
$$

where $\sigma(x)$ id defined by (1.14) satisfying (2.28), $C_{5}>0$ is a positive constant independent of time, and $\delta_{1}:=\left\|\sigma^{-\frac{\lambda}{2}}\left(\rho_{0}-\widetilde{\rho}, u_{0}-\widetilde{u}, n_{0}-\widetilde{n}, v_{0}-\widetilde{v}\right)\right\|_{H^{1}}$ is a non-negative constant.

Remark 1.1. For $M_{+}>1$, the nonlinear stability of the steady-state $(\widetilde{\rho}, \widetilde{u}, \widetilde{n}, \widetilde{v})$ for the outflow problem (1.1)-(1.5) with an exponential time decay rate can be gained. Indeed, assume that $M_{+}>1, u_{+}<0$ and a certain positive constant $\lambda>0$ hold. For a certain positive constant $\kappa \in(0, \lambda]$, there exists a small positive constant $\varepsilon_{0}>0$ such that if $\left(e^{\frac{\lambda}{2} x}\left(n_{0}-\widetilde{n}, \rho_{0}-\widetilde{\rho}, u_{0}-\widetilde{u}, n_{0}-\widetilde{n}, v_{0}-\widetilde{v}\right)\right) \in L^{2}\left(\mathbb{R}_{+}\right)$and

$$
\left.\|\left(\rho_{0}-\widetilde{\rho}, u_{0}-\widetilde{u}, n_{0}-\widetilde{n}, v_{0}-\widetilde{v}\right)\right) \|_{H^{1}}+\delta \leq \varepsilon_{0},
$$

then then the solution $(\rho, u, n, v)(t, x)$ to the IBVP (1.1)-(1.5) satisfies

$$
\|(\rho-\widetilde{\rho}, u-\widetilde{u}, n-\widetilde{n}, v-\widetilde{v})(t)\|_{H^{1}} \leq C_{6} \delta_{2} e^{-\frac{\kappa_{1}}{2} t},
$$

where $C_{6}>0$ and $\kappa_{1} \ll \kappa$ are positive constants independent of time, and $\delta_{2}:=\|\left(\rho_{0}-\widetilde{\rho}, u_{0}-\widetilde{u}, n_{0}-\right.$ $\left.\widetilde{n}, v_{0}-\widetilde{v}\right)\left\|_{H^{1}}+\right\| e^{\frac{\lambda}{2} x}\left(\rho_{0}-\widetilde{\rho}, u_{0}-\widetilde{u}, n_{0}-\widetilde{n}, v_{0}-\widetilde{v}\right) \|_{L^{2}}$ is a non-negative constant.

The proof of (1.22) can be obtained by similar arguments as (1.18). The details are omitted.

We explain the strategies to prove above main Theorems. The system (1.1)-(1.5) can be viewed as the two Navier-Stokes systems coupled by the drag force. On the one hand, compared with the single Navier-Stokes system, it is more difficult to prove the existence of the steady-states to IBVP (1.1)-(1.5). Indeed, it is easy to check that there is no trivial steady-states which are constructed by steady-states to the Navier-Stokes system compared with $[4,9,33,40]$. In order to use the center manifold theory [1], we need to introduce the new variable $\widetilde{w}:=\widetilde{u}_{x}$, get the estimates $\left|\widetilde{u}_{x}(0)\right| \leq C\left|u_{-}-u_{+}\right|$in Lemma 2.1, and reformulate the system (1.6) into the first-order autonomous system (2.9). On the other hand, the 
linearized reformulated system has at least one eigenvalue with negative real part due to the effect of the drag force. Thus, we obtain the existence of the steady-states for supersonic, sonic and subsonic cases in Theorem 1.1. The steady-states exist for the subsonic case, which is different from the single Navier-Stokes system [19].

To prove the nonlinear stability of the steady-states in Theorem 1.2, it is necessary to obtain the uniform estimates on the perturbations around steady-states $(\phi, \psi, \bar{\phi}, \bar{\psi})$. In fact, compared with the single Navier-Stokes system, it is more difficult to get the uniform estimates for sonic flow due to the drag force. Indeed, the bad term

$$
\int R_{2} d x=\int \phi \psi \frac{\left(\widetilde{\rho} \widetilde{u}^{2}+p_{1}(\widetilde{\rho})\right)_{x}}{\widetilde{\rho}}+\bar{\phi} \bar{\psi} \frac{\left(\widetilde{n} \widetilde{v}^{2}+p_{2}(\widetilde{n})\right)_{x}}{\widetilde{n}} d x=\int \phi \psi \frac{\mu \widetilde{u}_{x x}+\widetilde{n}(\widetilde{v}-\widetilde{u})}{\widetilde{\rho}}+\bar{\phi} \bar{\psi} \frac{(\widetilde{n} \widetilde{v})_{x}-\widetilde{n}(\widetilde{v}-\widetilde{u})}{\widetilde{n}} d x
$$

can be controlled by the good term

$$
\int R_{1} d x=\int\left[\rho \psi^{2}+p_{1}(\rho)-p_{1}(\widetilde{\rho})-p_{1}^{\prime}(\widetilde{\rho}) \phi\right] \widetilde{u}_{x}+\left[n \bar{\psi}^{2}+p_{2}(n)-p_{2}(\widetilde{n})-p_{2}^{\prime}(\widetilde{n}) \bar{\phi}\right] \widetilde{v}_{x} d x
$$

under the assumption $\left|p_{1}^{\prime}\left(\rho_{+}\right)-p_{2}^{\prime}\left(n_{+}\right)\right| \leq \sqrt{2} \min \left\{\left(1+\frac{\rho_{+}}{n_{+}}\right)\left[(\gamma-1) p_{1}^{\prime}\left(\rho_{+}\right)\right]^{\frac{1}{2}},\left(1+\frac{n_{+}}{\rho_{+}}\right)\left[(\alpha-1) p_{2}^{\prime}\left(n_{+}\right)\right]^{\frac{1}{2}}\right\}$.

With the weighted energy method, we get the exponential or algebraic time decay rates for the supersonic case. For sonic case, we obtain the existence and the algebraic time decay rates of the solution to IBVP (1.1)-(1.5) without the restriction $\left|p_{1}^{\prime}\left(\rho_{+}\right)-p_{2}^{\prime}\left(n_{+}\right)\right| \leq \sqrt{2} \min \left\{\left(1+\frac{\rho_{+}}{n_{+}}\right)\left[(\gamma-1) p_{1}^{\prime}\left(\rho_{+}\right)\right]^{\frac{1}{2}},(1+\right.$ $\left.\left.\frac{n_{+}}{\rho_{+}}\right)\left[(\alpha-1) p_{2}^{\prime}\left(n_{+}\right)\right]^{\frac{1}{2}}\right\}$ by the weighted energy method. It is difficult to obtain the convergence rate for the supersonic case by the ideas used in compressible Navier-Stokes equations [28] except for $p_{1}^{\prime}\left(\rho_{+}\right)=p_{2}^{\prime}\left(n_{+}\right)$. And for the sonic case, the ideas in compressible Navier-Stokes equations [28] can't work except for the special case $n_{+}=\rho_{+}, \mu=n_{+}, p_{1}^{\prime}\left(\rho_{+}\right)=p_{2}^{\prime}\left(n_{+}\right)$and $\gamma=\alpha$. To get decay rates for both supersonic and sonic case, we make full use of the drag force term. Indeed, one important observation is decomposing $\psi$ as

$$
\psi=\bar{\psi}+(\psi-\bar{\psi})
$$

in basic energy estimates for both supersonic and sonic cases. For the sonic case, inspired by [27,28], we take the linear coordinate transformation

$$
\left(\begin{array}{l}
\phi \\
\bar{\phi} \\
\bar{\psi}
\end{array}\right)=\boldsymbol{P}\left(\begin{array}{l}
\hat{\rho} \\
\hat{n} \\
\hat{v}
\end{array}\right)
$$

with a invertible matrix $\boldsymbol{P}$. With the help of $\psi=\bar{\psi}+(\psi-\bar{\psi})$, the coordinate transformation and (1.13), we can get the good term for $\nu \in(0,3]$ that

$$
a \frac{A_{1} \gamma(\gamma+1) \rho_{+}^{\gamma}+A_{2} \alpha(\alpha+1) n_{+}^{\alpha+1}}{2\left|u_{+}\right|^{2}}\left[1+\nu-\frac{\nu(\nu-1)}{2\left(1+b^{2}\right)}\right] \int \sigma^{-(\nu-2)} \hat{v}^{2} d x+c \int \sigma^{-(\nu-1)}\left(\hat{\rho}^{2}+\hat{n}^{2}\right) d x,
$$

to control the bad term

$$
\int \sigma^{-\nu} R_{2}=\int \sigma^{-\nu}\left[\phi \psi \frac{\left(\widetilde{\rho} \widetilde{u}^{2}+p_{1}(\widetilde{\rho})\right)_{x}}{\widetilde{\rho}}+\bar{\phi} \bar{\psi} \frac{\left(\widetilde{n} \widetilde{v}^{2}+p_{2}(\widetilde{n})\right)_{x}}{\widetilde{n}}\right] d x
$$


where the constants $a, b$ are defined by (2.22) and the space weight function $\sigma \geq 0$ satisfies (1.14) and (2.28). However, it is more difficult to get decay rates for $p_{1}^{\prime}\left(\rho_{+}\right) \neq p_{2}^{\prime}\left(n_{+}\right)$due to the term

$$
\begin{aligned}
& a \nu \int \sigma^{-(\nu-1)}\left[-\rho_{+} u_{+} \bar{\psi}(\psi-\bar{\psi})-A_{1} \gamma \rho_{+}^{\gamma-1} \phi(\psi-\bar{\psi})\right] d x \\
\geq & a \nu \int \sigma^{-(\nu-1)} \frac{\rho_{+}\left(u_{+}^{2}-A_{1} \gamma \rho_{+}^{\gamma-1}\right)}{\left|u_{+}\right|} \hat{v}(\psi-\bar{\psi}) d x-C \delta^{\frac{1}{2}}\left[\int \sigma^{-(\nu-1)}\left(\hat{\rho}^{2}+\hat{n}^{2}\right) d x+\int \sigma^{-(\nu-2)} \hat{v}^{2} d x\right],
\end{aligned}
$$

which is caused by the decomposition $\psi=\bar{\psi}+(\psi-\bar{\psi})$. To overcome the difficulty, it is necessary to control the bad term by using good terms for $\nu \in(0,3]$ that

$$
\begin{aligned}
& a \frac{A_{1} \gamma(\gamma+1) \rho_{+}^{\gamma}+A_{2} \alpha(\alpha+1) n_{+}^{\alpha+1}}{2\left|u_{+}\right|^{2}}\left[1+\nu-\frac{\nu(\nu-1)}{2\left(1+b^{2}\right)}\right] \int \sigma^{-(\nu-2)} \hat{v}^{2} d x+n_{+} \int \sigma^{-\nu}(\psi-\bar{\psi})^{2} d x \\
& +c \int \sigma^{-(\nu-1)}\left(\hat{\rho}^{2}+\hat{n}^{2}\right) d x,
\end{aligned}
$$

where $a>0, b \geq 0$ are constants defined by (2.22), and the space weight function $\sigma \geq 0$ satisfies (1.14) and (2.28).

Notation. We denote by $\|\cdot\|_{L^{p}}$ the norm of the usual Lebesgue space $L^{p}=L^{p}\left(\mathbb{R}_{+}\right), 1 \leq p \leq \infty$. And if $p=2$, we write $\|\cdot\|_{L^{p}\left(\mathbb{R}_{+}\right)}=\|\cdot\| \cdot H^{s}\left(\mathbb{R}_{+}\right)$stands for the standard $s$-th Sobolev space over $\mathbb{R}_{+}$equipped with its norm

$$
\|f\|_{H^{s}\left(\mathbb{R}_{+}\right)}=\|f\|_{s}=\left(\sum_{i=0}^{s}\left\|\partial^{i} f\right\|^{2}\right)^{\frac{1}{2}} .
$$

$C\left([0, T] ; H^{1}\left(\mathbb{R}_{+}\right)\right)$represents the space of continuous functions on the interval $[0, T]$ with values in $H^{s}\left(\mathbb{R}_{+}\right) . \quad L^{2}([0, T] ; \mathcal{B})$ denotes the space of $L^{2}$ functions on the interval $[0, T]$ with values in the Banach space $\mathcal{B}$. For a scalar function $W(x)>0$, the weighted $L^{2}\left(\mathbb{R}_{+}\right)$and $H^{1}\left(\mathbb{R}_{+}\right)$spaces are defined as follows:

$$
\begin{aligned}
& L_{W}^{2}\left(\mathbb{R}_{+}\right):=\left\{f \in L^{2}\left(\mathbb{R}_{+}\right) \mid\|f\|_{L_{W}^{2}}:=\left(\int_{\mathbb{R}_{+}} W(x) f^{2} d x\right)^{\frac{1}{2}}<+\infty\right\}, \\
& H_{W}^{1}\left(\mathbb{R}_{+}\right):=\left\{f \in H^{1}\left(\mathbb{R}_{+}\right) \mid\|f\|_{H_{W}^{1}}:=\left(\sum_{i=0}^{1}\left\|\partial_{i} f\right\|_{L_{W}^{2}}^{2}\right)^{\frac{1}{2}}<+\infty\right\},
\end{aligned}
$$

For a scalar function $W_{a, \nu}:=(1+x)^{\nu}$ with $\nu \geq 0$, we denote $\|f\|_{a, \nu}:=\left\|(1+x)^{\frac{\nu}{2}} f\right\|$.

The rest of this paper will be organized as follows. We prove the existence and uniqueness of the steady-states in Section 2, get the nonlinear stability of the steady-states in Section 3 for supersonic, sonic and subsonic cases, and obtain the convergence rates of the steady-states for supersonic flow in Subsection 4.1 and sonic flow in Subsection 4.2.

\section{Existence of Stationary Solution}

We prove Theorem 1.1 on the the existence and uniqueness of the stationary solution to (1.6)-(1.7) with $u_{+}<0$ and $\delta$ sufficiently small as follows. First, to apply the center manifold theory [1], it is necessary to get the bounds of $\widetilde{u}_{x}(0)$ or $\widetilde{v}_{x}(0)$. 
Lemma 2.1. Assume that $u_{+}<0$ and $\delta=\left|u_{-}-u_{+}\right|$hold with $\delta$ sufficiently small. Let $(\widetilde{\rho}, \widetilde{u}, \widetilde{n}, \widetilde{v})$ be the solution to the BVP (1.6)-(1.7). Then we have

$$
\left|\widetilde{u}_{x}(0)\right| \leq C\left|u_{-}-u_{+}\right|, \quad\left|\widetilde{v}_{x}(0)\right| \leq C\left|u_{-}-u_{+}\right|
$$

where $C>0$ is a positive constant.

Proof. Due to $\widetilde{\rho}=\frac{\rho_{+} u_{+}}{\widetilde{u}}$ and $\widetilde{n}=\frac{n_{+} u_{+}}{\widetilde{v}}$, we can obtain

$$
\left\{\begin{array}{l}
\left(\rho_{+} u_{+} \widetilde{u}+A_{1} \rho_{+}^{\gamma} u_{+}^{\gamma} \widetilde{u}^{-\gamma}\right)_{x}=\left(\mu \widetilde{u}_{x}\right)_{x}+\frac{n_{+} u_{+}}{\widetilde{v}}(\widetilde{v}-\widetilde{u}), \\
\left(n_{+} u_{+} \widetilde{v}+A_{2} n_{+}^{\alpha} u_{+}^{\alpha} \widetilde{v}^{-\alpha}\right)_{x}=\left(n_{+} u_{+} \frac{\widetilde{v}_{x}}{\widetilde{v}}\right)_{x}-\frac{n_{+} u_{+}}{\widetilde{v}}(\widetilde{v}-\widetilde{u}) .
\end{array}\right.
$$

Adding $(2.2)_{1}$ to $(2.2)_{2}$ and integrating the resulted equation over $(0,+\infty)$ lead to

$$
\mu \widetilde{u}_{x}(0)+\frac{n_{+} u_{+}}{u_{-}} \widetilde{v}_{x}(0)=\frac{1}{u_{+}}\left[\left(\rho_{+}+n_{+}\right) u_{+}^{2}-\left(A_{1} \gamma \rho_{+}^{\gamma}+A_{2} \alpha n_{+}^{\alpha}\right)\right]\left(u_{-}-u_{+}\right)+O\left(\left|u_{-}-u_{+}\right|^{2}\right) .
$$

Multiplying $(2.2)_{1}$ by $\widetilde{u},(2.2)_{2}$ by $\widetilde{v}$, respectively, then adding them together and integrating the resulted equation over $(0,+\infty)$, we can gain

$$
\begin{aligned}
& \int_{0}^{+\infty}\left(\mu \widetilde{u}_{x}^{2}+n_{+} u_{+} \frac{\widetilde{v}_{x}^{2}}{\widetilde{v}}\right) d x+\int_{0}^{+\infty} \frac{n_{+} u_{+}}{\widetilde{v}}(\widetilde{v}-\widetilde{u})^{2} d x \\
& =-\left[\mu \widetilde{u}_{x}(0)+n_{+} u_{+} \frac{\widetilde{v}_{x}(0)}{u_{-}}\right]+\left[\left(\rho_{+}+n_{+}\right) u_{+}^{2}-\left(A_{1} \gamma \rho_{+}^{\gamma}+A_{2} \alpha n_{+}^{\alpha}\right)\right]\left(u_{-}-u_{+}\right)+O\left(\left|u_{-}-u_{+}\right|^{2}\right) \\
& =O\left(\left|u_{-}-u_{+}\right|^{2}\right),
\end{aligned}
$$

where we have used (2.3). Multiplying $(2.2)_{2}$ by $\frac{\widetilde{v}_{x}}{\widetilde{v}}$ and then integrating the resulted equation over $(0, \infty)$ yield

$$
-n_{+} u_{+} \frac{\widetilde{v}_{x}^{2}(0)}{2 u_{-}^{2}}+\int_{0}^{+\infty} A_{2} \alpha n_{+}^{\alpha} \frac{u_{+}^{\alpha}}{\widetilde{v}^{\alpha+2}} \widetilde{v}_{x}^{2} d x=\int_{0}^{+\infty} \frac{n_{+} u_{+}}{\widetilde{v}}(\widetilde{v}-\widetilde{u}) \frac{\widetilde{v}_{x}}{\widetilde{v}} d x+\int_{0}^{+\infty} n_{+} u_{+} \frac{\widetilde{v}_{x}^{2}}{\widetilde{v}} d x
$$

We estimate terms in the right hand side of (2.5). Using $\inf _{x \in \mathbb{R}_{+}} \widetilde{n}>0, \alpha \geq 1$ and (2.3), we have

$$
\begin{aligned}
& \int_{0}^{+\infty} \frac{n_{+} u_{+}}{\widetilde{v}}(\widetilde{v}-\widetilde{u}) \frac{\widetilde{v}_{x}}{\widetilde{v}} d x+\int_{0}^{+\infty} n_{+} u_{+} \frac{\widetilde{v}_{x}^{2}}{\widetilde{v}} d x \\
\leq & C \int_{0}^{+\infty} \frac{n_{+} u_{+}}{\widetilde{v}}(\widetilde{v}-\widetilde{u})^{2} d x+\frac{1}{4} \int_{0}^{+\infty} A_{2} \alpha n_{+}^{\alpha} \frac{u_{+}^{\alpha}}{\widetilde{v}^{\alpha+2}} \widetilde{v}_{x}^{2} d x \\
\leq & C\left|u_{-}-u_{+}\right|^{2}+\frac{1}{4} \int_{0}^{+\infty} A_{2} \alpha n_{+}^{\alpha} \frac{u_{+}^{\alpha}}{\widetilde{v}^{\alpha+2}} \widetilde{v}_{x}^{2} d x .
\end{aligned}
$$

By using (2.5) and (2.6), we can get

$$
\left|\widetilde{v}_{x}(0)\right| \leq C\left|u_{-}-u_{+}\right|, \quad\left|\widetilde{u}_{x}(0)\right| \leq C\left|u_{-}-u_{+}\right| .
$$


Then, with the above lemma, we can prove Theorem 1.1. For $(1.6)_{2}$ and $(1.6)_{4}$, using $\widetilde{\rho}=\frac{\rho_{+} u_{+}}{\widetilde{u}}$, $\widetilde{n}=\frac{n_{+} u_{+}}{\widetilde{v}}$ and integrating the summation of $(1.6)_{2},(1.6)_{4}$ over $(x,+\infty)$, we have

$$
\left\{\begin{array}{l}
\widetilde{v}_{x}=\frac{\widetilde{v}}{n_{+} u_{+}}\left[\rho_{+} u_{+}\left(\widetilde{u}-u_{+}\right)+A_{1} \rho_{+}^{\gamma}\left(\frac{u_{+}^{\gamma}}{\widetilde{u}^{\gamma}}-1\right)+n_{+} u_{+}\left(\widetilde{v}-u_{+}\right)+A_{2} n_{+}^{\alpha}\left(\frac{u_{+}^{\alpha}}{\widetilde{v}^{\alpha}}-1\right)-\mu \widetilde{u}_{x}\right], \\
\widetilde{u}_{x x}=\frac{1}{\mu}\left[\left(\rho_{+} u_{+}-A_{1} \rho_{+}^{\gamma} u_{+}^{\gamma} \widetilde{u}^{-\gamma-1}\right) \widetilde{u}_{x}-n_{+} u_{+}\left(1-\frac{\widetilde{u}}{\widetilde{v}}\right)\right] .
\end{array}\right.
$$

Define $\widetilde{w}:=\widetilde{u}_{x}$ and $\bar{U}:=(\bar{u}, \bar{w}, \bar{v})^{\mathrm{T}}:=\left(\widetilde{u}-u_{+}, \widetilde{w}, \widetilde{v}-u_{+}\right)^{\mathrm{T}}$. The system (2.8) can be reformulated into the autonomous first-order system as follows

$$
\left\{\begin{array}{l}
\bar{U}_{x}=\boldsymbol{J}_{+} \bar{U}+\left(0, \bar{g}_{2}(\bar{U}), \bar{g}_{3}(\bar{U})\right)^{\mathrm{T}}, \\
\bar{U}_{-}:=(\bar{u}, \bar{w}, \bar{v})^{\mathrm{T}}(0)=\left(u_{-}-u_{+}, \widetilde{u}_{x}(0), u_{-}-u_{+}\right)^{\mathrm{T}}, \lim _{x \rightarrow \infty} \bar{U}^{\mathrm{T}}=(0,0,0),
\end{array}\right.
$$

where

$$
\begin{aligned}
\boldsymbol{J}_{+}= & \left(\begin{array}{ccc}
0 & 1 & 0 \\
\frac{n_{+}}{\mu} & \frac{\rho_{+} u_{+}^{2}-A_{1} \gamma \rho_{+}^{\gamma}}{\mu u_{+}} & -\frac{n_{+}}{\mu} \\
\frac{\rho_{+} u_{+}^{2}-A_{1} \gamma \rho_{+}^{\gamma}}{n_{+} u_{+}} & -\frac{\mu}{n_{+}} & \frac{n_{+} u_{+}^{2}-A_{2} \alpha n_{+}^{\alpha}}{n_{+} u_{+}}
\end{array}\right), \\
\bar{g}_{2}(\bar{U})= & \frac{1}{2}\left(2 \frac{n_{+}}{\mu} \frac{1}{u_{+}} \bar{v}^{2}-2 \frac{n_{+}}{\mu} \frac{1}{u_{+}} \bar{u} \bar{v}+2 \frac{A_{1} \gamma(\gamma+1) \rho_{+}^{\gamma}}{\mu u_{+}^{2}} \bar{w} \bar{u}\right)+O\left(|\bar{U}|^{3}\right), \\
\bar{g}_{3}(\bar{U})= & \frac{1}{2}\left[\frac{A_{1} \gamma(\gamma+1) \rho_{+}^{\gamma}}{n_{+} u_{+}^{2}} \bar{u}^{2}+2 \frac{\rho_{+} u_{+}^{2}-A_{1} \gamma \rho_{+}^{\gamma}}{n_{+} u_{+}^{2}} \bar{v} \bar{u}+\left(2 \frac{n_{+} u_{+}^{2}-A_{2} \alpha n_{+}^{\alpha}}{n_{+} u_{+}^{2}}\right.\right. \\
& \left.\left.+\frac{A_{2} \alpha(\alpha+1) n_{+}^{\alpha}}{n_{+} u_{+}^{2}}\right) \bar{v}^{2}-2 \frac{\mu}{n_{+} u_{+}} \bar{w} \bar{v}\right]+O\left(|\bar{U}|^{3}\right) .
\end{aligned}
$$

Three eigenvalues $\lambda_{1}, \lambda_{2}, \lambda_{3}$ of matrix $\boldsymbol{J}_{+}$satisfy

$$
\left\{\begin{array}{l}
\lambda_{1} \lambda_{2} \lambda_{3}=-\frac{\left(n_{+}+\rho_{+}\right) u_{+}^{2}-\left(A_{1} \gamma \rho_{+}^{\gamma}+A_{2} \alpha n_{+}^{\alpha}\right)}{\mu u_{+}}, \\
\lambda_{1}+\lambda_{2}+\lambda_{3}=\frac{\rho_{+} u_{+}^{2}-A_{1} \gamma \rho_{+}^{\gamma}}{\mu u_{+}}+\frac{n_{+} u_{+}^{2}-A_{2} \alpha n_{+}^{\alpha}}{n_{+} u_{+}}, \\
\lambda_{1} \lambda_{2}+\lambda_{1} \lambda_{3}+\lambda_{2} \lambda_{3}=\rho_{+} \frac{\left(u_{+}^{2}-A_{1} \gamma \rho_{+}^{\gamma-1}\right)\left(u_{+}^{2}-A_{2} \alpha n_{+}^{\alpha-1}\right)}{\mu u_{+}^{2}}-1-\frac{n_{+}}{\mu} .
\end{array}\right.
$$

If $M_{+}>1$, it is easy to obtain $\lambda_{1} \lambda_{2} \lambda_{3}>0$ and $u_{+}^{2}>\min \left\{A_{1} \gamma \rho_{+}^{\gamma-1}, A_{2} \alpha n_{+}^{\alpha-1}\right\}$. Without loss of generality, we assume $A_{1} \gamma \rho_{+}^{\gamma-1} \geq A_{2} \alpha n_{+}^{\alpha-1}$. Moreover, we have

$$
\lambda_{1}+\lambda_{2}+\lambda_{3}<0 \text { for } u_{+}^{2}>A_{1} \gamma \rho_{+}^{\gamma-1}, \quad \lambda_{1} \lambda_{2}+\lambda_{1} \lambda_{3}+\lambda_{2} \lambda_{3}<0 \text { for } A_{2} \alpha n_{+}^{\alpha-1} \leq u_{+}^{2} \leq A_{1} \gamma \rho_{+}^{\gamma-1},
$$

which can imply $\operatorname{Re} \lambda_{1}<0, \operatorname{Re} \lambda_{2}<0$ and $\lambda_{3}>0$ for $M_{+}>1$. Using similar arguments, we have the following cases:

$$
\left\{\begin{array}{l}
\text { if } M_{+}>1, \text { then } \operatorname{Re} \lambda_{1}<0, \operatorname{Re} \lambda_{2}<0, \lambda_{3}>0, \\
\text { if } M_{+}<1, \text { then } \operatorname{Re} \lambda_{1}>0, \operatorname{Re} \lambda_{2}>0, \lambda_{3}<0 \\
\text { if } M_{+}=1, \text { then } \lambda_{1}>0, \lambda_{2}<0, \lambda_{3}=0 .
\end{array}\right.
$$


Then, applying the center manifold theory [1], it is not difficult to show the case $M_{+} \neq 1$ in Theorem 1.1 if $\delta$ is small. Finally, we prove case $M_{+}=1$ in Theorem 1.1 which implies $\lambda_{1}>0, \lambda_{2}<0, \lambda_{3}=0$. The eigenvectors $r_{1}, r_{2}, r_{3}$ of $\lambda_{1}, \lambda_{2}, \lambda_{3}$ are obtained respectively as follows

$$
r_{1}=\left(\begin{array}{c}
1 \\
\lambda_{1} \\
-\frac{\mu}{n_{+}}\left(\lambda_{1}^{2}-\frac{\rho_{+} u_{+}^{2}-A_{1} \gamma \rho_{+}^{\gamma}}{\mu u_{+}} \lambda_{1}\right)+1
\end{array}\right), r_{2}=\left(\begin{array}{c}
1 \\
\lambda_{2} \\
-\frac{\mu}{n_{+}}\left(\lambda_{2}^{2}-\frac{\rho_{+} u_{+}^{2}-A_{1} \gamma \rho_{+}^{\gamma}}{\mu u_{+}} \lambda_{2}\right)+1
\end{array}\right), r_{3}=\left(\begin{array}{l}
1 \\
0 \\
1
\end{array}\right) .
$$

Define the matrix $\boldsymbol{P}_{\mathbf{1}}:=\left[r_{1}, r_{2}, r_{3}\right]$ and take a linear change of coordinate $Z:=\left(z_{1}, z_{2}, z_{3}\right)^{\mathrm{T}}=\boldsymbol{P}_{\mathbf{1}}{ }^{-1} \bar{U}$. With (2.11) and (2.12), the system (2.9) can be reformulated as follows

$$
\left\{\begin{array}{l}
\frac{d}{d x}\left(\begin{array}{c}
z_{1} \\
z_{2} \\
z_{3}
\end{array}\right)=\left(\begin{array}{ccc}
\lambda_{1} & * & 0 \\
0 & \lambda_{2} & 0 \\
0 & 0 & \lambda_{3}
\end{array}\right)\left(\begin{array}{l}
z_{1} \\
z_{2} \\
z_{3}
\end{array}\right)+\left(\begin{array}{l}
g_{1}\left(z_{1}, z_{2}, z_{3}\right) \\
g_{2}\left(z_{1}, z_{2}, z_{3}\right) \\
g_{3}\left(z_{1}, z_{2}, z_{3}\right)
\end{array}\right) \\
\left(z_{1}, z_{2}, z_{3}\right)(0)=\left(z_{1-}, z_{2-}, z_{3-}\right)=\left(\boldsymbol{P}_{1}^{-1} \bar{U}_{-}\right)^{\mathrm{T}}, \quad \lim _{x \rightarrow \infty}\left(z_{1}, z_{2}, z_{3}\right)=(0,0,0),
\end{array}\right.
$$

where nonlinear functions $g_{i}(i=1,2,3)$ are denoted by

$$
\left(\begin{array}{l}
g_{1}\left(z_{1}, z_{2}, z_{3}\right) \\
g_{2}\left(z_{1}, z_{2}, z_{3}\right) \\
g_{3}\left(z_{1}, z_{2}, z_{3}\right)
\end{array}\right)=\boldsymbol{P}_{\mathbf{1}}^{-1}\left(\begin{array}{c}
0 \\
\bar{g}_{2}(\bar{u}, \bar{w}, \bar{v}) \\
\bar{g}_{3}(\bar{u}, \bar{w}, \bar{v})
\end{array}\right)
$$

With the help of the manifold theory [1], there exist a local center manifold $W^{c}(0,0,0)$ and a local stable manifold $W_{3}^{s}(0,0,0)$

$$
\begin{aligned}
& W^{c}(0,0,0)=\left\{\left(z_{1}, z_{2}, z_{3}\right)\left|z_{1}=f_{1}^{c}\left(z_{3}\right), z_{2}=f_{2}^{c}\left(z_{3}\right),\right| z_{3} \mid \text { sufficient small }\right\} \\
& W_{3}^{s}(0,0,0)=\left\{\left(z_{1}, z_{2}, z_{3}\right)\left|z_{1}=f_{1}^{s}\left(z_{2}\right), z_{3}=f_{2}^{s}\left(z_{2}\right),\right| z_{2} \mid \text { sufficient small }\right\}
\end{aligned}
$$

where $f_{i}^{c}, f_{i}^{s}, i=1,2$ are smooth functions and $f_{i}^{c}(0)=0, D f_{i}^{c}(0)=0, f_{i}^{s}(0)=0, D f_{i}^{s}(0)=0, i=1,2$. Using $\bar{U}=P Z,(2.12),(2.18)$, we can gain

$$
\bar{g}_{3}\left(z_{3}\right)=a z_{3}^{2}+O\left(\left|z_{1}\right|^{2}+\left|z_{2}\right|^{2}+\left|z_{3}\right|^{3}+\left|z_{1} z_{3}\right|+\left|z_{2} z_{3}\right|\right),
$$

where

$$
a=\frac{A_{1} \gamma(\gamma+1) \rho_{+}^{\gamma}+A_{2} \alpha(\alpha+1) n_{+}^{\alpha}}{2 u_{+}^{2}\left(1+b^{2}\right)\left(\mu+n_{+}\right)}, \quad b:=\frac{\rho_{+}\left(u_{+}^{2}-p_{1}^{\prime}\left(\rho_{+}\right)\right)}{\left|u_{+}\right| \sqrt{\left(\mu+n_{+}\right) n_{+}}} .
$$

Therefore, the system (2.17) can be reformulated as follows

$$
\left\{\begin{array}{l}
z_{1 x}=\lambda_{1} z_{1}+O\left(|Z|^{2}\right), \\
z_{2 x}=\lambda_{2} z_{2}+O\left(|Z|^{2}\right), \\
z_{3 x}=a z_{3}^{2}+O\left(\left|z_{1}\right|^{2}+\left|z_{2}\right|^{2}+\left|z_{3}\right|^{3}+\left|z_{1} z_{3}\right|+\left|z_{2} z_{3}\right|\right), \\
\left(z_{1}, z_{2}, z_{3}\right)(0):=\left(z_{1-}, z_{2-}, z_{3-}\right)=\left(\boldsymbol{P}_{1}^{-1} \bar{U}_{-}\right)^{\mathrm{T}}, \lim _{x \rightarrow \infty}\left(z_{1}, z_{2}, z_{3}\right)=(0,0,0) .
\end{array}\right.
$$


Let $\sigma_{1}(x)$ be a solution to $(2.23)_{1}$ restricted on the local center manifold satisfying the equation

$$
\sigma_{1 x}=a \sigma_{1}^{2}+O\left(\sigma_{1}^{3}\right), \quad \sigma_{1}(x) \rightarrow 0 \text { as } x \rightarrow+\infty .
$$

which implies that there exists the monotonically increasing solution $\sigma_{1}(x)<0$ to $(2.24)$ for $\sigma_{1}(0)<0$ and $\left|\sigma_{1}(0)\right|$ sufficiently small. Therefore, if the initial data $\left(z_{1-}, z_{2-}, z_{3-}\right)$ belongs to the region $\mathcal{M} \in \mathbb{R}^{3}$ associated to the local stable manifold and the local center manifold, then we have

$$
\left\{\begin{array}{l}
z_{i}=O\left(\sigma_{1}^{2}\right)+O\left(\delta e^{-c x}\right), i=1,2 \\
z_{3}=\sigma_{1}+O\left(\delta e^{-c x}\right)
\end{array}\right.
$$

with $z_{3-}<0$, the smallness of $\left|\left(z_{1-}, z_{2-}, z_{3-}\right)\right|$ and

$$
c \frac{\delta}{1+\delta x} \leq\left|\sigma_{1}\right| \leq C \frac{\delta}{1+\delta x}, \quad\left|\partial^{k} \sigma_{1}\right| \leq C \frac{\delta^{k+1}}{(1+\delta x)^{k+1}}, \quad C>0, \quad k=0,1,2,3 .
$$

Due to $\sigma_{1}(x) \leq 0$, we define

$$
\sigma(x):=-\sigma_{1},
$$

which satisfies

$$
\sigma_{x}=-a \sigma^{2}+O\left(|\sigma|^{3}\right), \quad \sigma \rightarrow 0 \text { as } x \rightarrow+\infty
$$

It is easy to get

$$
\left|\partial_{x}^{k}\left(\widetilde{\rho}-\rho_{+}, \widetilde{u}-u_{+}, \widetilde{n}-n_{+}, \widetilde{v}-u_{+}\right)\right| \leq C \frac{\delta^{k+1}}{(1+\delta x)^{k+1}}, \quad C>0, \quad k=0,1,2,3,
$$

and

$$
\left(\widetilde{u}-u_{+}, \widetilde{v}-u_{+}\right)=(-\sigma(x),-\sigma(x))+O\left(|\sigma(x)|^{2}\right), \quad\left(\widetilde{u}_{x}, \widetilde{v}_{x}\right)=\left(a \sigma^{2}(x), a \sigma^{2}(x)\right)+O\left(|\sigma|^{3}\right),
$$

with the help of $\bar{U}=\boldsymbol{P} Z$ and (2.16).

\section{Nonlinear stability of steady-states}

The function space $Y(0, T)$ for $T>0$ is denoted by

$$
\begin{aligned}
Y(0, T):=\{(\phi, \psi, \bar{\phi}, \bar{\psi}) \mid & (\phi, \psi, \bar{\phi}, \bar{\psi}) \in C\left([0, T] ; H^{1}\right), \\
& \left.\left(\phi_{x}, \bar{\phi}_{x}\right) \in L^{2}\left([0, T] ; L^{2}\right),\left(\psi_{x}, \bar{\psi}_{x}\right) \in L^{2}\left([0, T] ; H^{1}\right)\right\} .
\end{aligned}
$$

Let

$$
\phi=\rho-\widetilde{\rho}, \quad \psi=u-\widetilde{u}, \quad \bar{\phi}=n-\widetilde{n}, \quad \bar{\psi}=v-\widetilde{v} .
$$


Then the perturbation $(\phi, \psi, \bar{\phi}, \bar{\psi})$ satisfies the following system

$$
\left\{\begin{array}{l}
\phi_{t}+u \phi_{x}+\rho \psi_{x}=-\left(\psi \widetilde{\rho}_{x}+\phi \widetilde{u}_{x}\right), \\
\psi_{t}+u \psi_{x}+\frac{p_{1}^{\prime}(\rho)}{\rho} \phi_{x}-\frac{\mu \psi_{x x}}{\rho}-\frac{n(\bar{\psi}-\psi)}{\rho}=F_{1}, \\
\bar{\phi}_{t}+v \bar{\phi}_{x}+n \bar{\psi}_{x}=-\left(\bar{\psi} \widetilde{n}_{x}+\bar{\phi} \widetilde{v}_{x}\right), \\
\bar{\psi}_{t}+v \bar{\psi}_{x}+\frac{p_{2}^{\prime}(n)}{n} \bar{\phi}_{x}-\frac{\left(n \bar{\psi}_{x}\right)_{x}}{n}+(\bar{\psi}-\psi)=F_{2},
\end{array}\right.
$$

where

$$
\begin{aligned}
& F_{1}=-\left[-\mu\left(\frac{1}{\rho}-\frac{1}{\widetilde{\rho}}\right) \widetilde{u}_{x x}+\psi \widetilde{u}_{x}+\left(\frac{p_{1}^{\prime}(\rho)}{\rho}-\frac{p_{1}^{\prime}(\widetilde{\rho})}{\widetilde{\rho}}\right) \widetilde{\rho}_{x}-\left(\frac{n}{\rho}-\frac{\widetilde{n}}{\widetilde{\rho}}\right)(\widetilde{v}-\widetilde{u})\right], \\
& F_{2}=-\left[-\left(\frac{1}{n}-\frac{1}{\widetilde{n}}\right)\left(\widetilde{n}_{v_{x}}\right)_{x}-\frac{\left(\bar{\phi} \widetilde{v}_{x}\right)_{x}}{n}+\bar{\psi} \widetilde{v}_{x}+\left(\frac{p_{2}^{\prime}(n)}{n}-\frac{p_{2}^{\prime}(\widetilde{n})}{\widetilde{n}}\right) \widetilde{n}_{x}\right] .
\end{aligned}
$$

The initial and boundary conditions to the system (3.3) satisfy

$$
\begin{gathered}
(\phi, \psi, \bar{\phi}, \bar{\psi})(0, x):=\left(\phi_{0}, \psi_{0}, \bar{\phi}_{0}, \bar{\psi}_{0}\right)=\left(\rho_{0}-\widetilde{\rho}, u_{0}-\widetilde{u}, n_{0}-\widetilde{n}, v_{0}-\widetilde{v}\right), \\
\lim _{x \rightarrow \infty}\left(\phi_{0}, \psi_{0}, \bar{\phi}_{0}, \bar{\psi}_{0}\right)=(0,0,0,0), \quad(\psi, \bar{\psi})(t, 0)=(0,0) .
\end{gathered}
$$

Proposition 3.1. Assume that the same assumptions in Theorem 1.2 hold. Let $(\phi, \psi, \bar{\phi}, \bar{\psi})$ be the solution to the problem (3.3)-(3.7) satisfying $(\phi, \psi, \bar{\phi}, \bar{\psi}) \in Y(0, T)$ for any time $T>0$. Then there exist positive constants $\varepsilon>0$ and $C>0$ independent of $T$ such that if

$$
\sup _{0 \leq t \leq T}\|(\phi, \psi, \bar{\phi}, \bar{\psi})\|_{1}+\delta \leq \varepsilon
$$

is satisfied, it holds for arbitrary $t \in[0, T]$ that

$$
\|(\phi, \psi, \bar{\phi}, \bar{\psi})\|_{1}+\int_{0}^{t}\left\|\left(\phi_{x}, \psi_{x}, \bar{\phi}_{x}, \bar{\psi}_{x}\right)\right\|^{2} d \tau+\int_{0}^{t}\left\|\left(\bar{\psi}-\psi, \psi_{x x}, \bar{\psi}_{x x}\right)\right\|^{2} d \tau \leq C\left\|\left(\phi_{0}, \psi_{0}, \bar{\phi}_{0}, \bar{\psi}_{0}\right)\right\|_{1}^{2} .
$$

Under the condition (3.8), it is easy to verify the following Sobolev inequality

$$
\|(\phi, \psi, \bar{\phi}, \bar{\psi})\|_{L^{\infty}} \leq\|(\phi, \psi, \bar{\phi}, \bar{\psi})\|_{H^{1}} \leq \sqrt{2} \varepsilon \text {. }
$$

Lemma 3.2 ( [19] ). The function $\psi(\cdot, t) \in H^{1}\left(\mathbb{R}_{+}\right)$satisfies for the constants $\delta>0$ and $c_{0}>0$ that

$$
\begin{aligned}
& \int_{0}^{\infty} \delta e^{-c_{0} x}|\psi|^{2} d x \leq C \delta\left(|\psi(0, t)|^{2}+\left\|\psi_{x}(t)\right\|^{2}\right), \\
& \int_{0}^{\infty} \frac{\delta^{j}}{(1+\delta x)^{j}}|\psi|^{2} d x \leq C \delta^{j-2}\left(|\psi(0, t)|^{2}+\left\|\psi_{x}(t)\right\|^{2}\right), \quad j>2,
\end{aligned}
$$

where $C>0$ is a positive constant independent of time.

With the above Lemma, we can gain the basic $L^{2}$ energy estimates of $(\phi, \psi, \bar{\phi}, \bar{\psi})$. 
Lemma 3.3. Under the same conditions in Proposition 3.1, then the solution $(\phi, \psi, \bar{\phi}, \bar{\psi})$ to the problem (3.3)-(3.7) satisfies for $t \in[0, T]$ that

$$
\begin{aligned}
& \|(\phi, \psi, \bar{\phi}, \bar{\psi})\|^{2}+\int_{0}^{t}\left\|\left(\psi_{x}, \bar{\psi}_{x}, \bar{\psi}-\psi\right)\right\|^{2} d \tau+\int_{0}^{t}|\phi(t, 0)|^{2}+|\bar{\phi}(t, 0)|^{2} d \tau \\
\leq & C\left\|\left(\phi_{0}, \psi_{0}, \bar{\phi}_{0}, \bar{\psi}_{0}\right)\right\|^{2}+C(\delta+\varepsilon) \int_{0}^{t}\left\|\left(\phi_{x}, \bar{\phi}_{x}\right)\right\|^{2} d \tau .
\end{aligned}
$$

Proof. Define

$$
\begin{array}{lll}
h_{1}(\rho)=\rho \int^{\rho} \frac{p_{1}(s)}{s^{2}} d s, & \Phi_{1}(\rho, \widetilde{\rho})=\int_{\widetilde{\rho}}^{\rho} \frac{p_{1}(s)-p_{1}(\widetilde{\rho})}{s^{2}} d s, & \mathcal{E}_{1}=\rho\left(\frac{\psi^{2}}{2}+\Phi_{1}\right), \\
h_{2}(n)=n \int^{n} \frac{p_{2}(s)}{s^{2}} d s, & \Phi_{2}(n, \widetilde{n})=\int_{\widetilde{n}}^{n} \frac{p_{2}(s)-p_{2}(\widetilde{n})}{s^{2}} d s, & \mathcal{E}_{2}=n\left(\frac{\bar{\psi}^{2}}{2}+\Phi_{2}\right) .
\end{array}
$$

Then, by (1.1) and (1.6), the direct computations lead to that

$$
\left(\mathcal{E}_{1}+\mathcal{E}_{2}\right)_{t}+\left(G_{1}+G_{2}\right)_{x}+n(\bar{\psi}-\psi)^{2}+\mu \psi_{x}^{2}+n \bar{\psi}_{x}^{2}+R_{1}+R_{2}=-R_{3},
$$

where

$$
\left\{\begin{array}{l}
G_{1}:=u \mathcal{E}_{1}+v \mathcal{E}_{2}+\left(p_{1}(\rho)-p_{1}(\widetilde{\rho})\right) \psi+\left(p_{2}(n)-p_{2}(\widetilde{n})\right) \bar{\psi} \\
G_{2}:=-\left(\mu \psi \psi_{x}+n \bar{\psi} \bar{\psi}_{x}+\bar{\phi} \bar{\psi} \widetilde{v}_{x}\right), \\
R_{1}:=\left[\rho \psi^{2}+p_{1}(\rho)-p_{1}(\widetilde{\rho})-p_{1}^{\prime}(\widetilde{\rho}) \phi\right] \widetilde{u}_{x}+\left[n \bar{\psi}^{2}+p_{2}(n)-p_{2}(\widetilde{n})-p_{2}^{\prime}(\widetilde{n}) \bar{\phi}\right] \widetilde{v}_{x}, \\
R_{2}:=\phi \psi \frac{\widetilde{\rho} \widetilde{u} \widetilde{u}_{x}+\left(p_{1}(\widetilde{\rho})\right)_{x}}{\widetilde{\rho}}+\bar{\phi} \bar{\psi} \frac{\widetilde{n} \widetilde{v} \widetilde{v}_{x}+\left(p_{2}(\widetilde{n})\right)_{x}}{\widetilde{n}}, \\
R_{3}:=\bar{\phi}(\bar{\psi}-\psi)(\widetilde{v}-\widetilde{u})+\bar{\phi} \bar{\psi}_{x} \widetilde{v}_{x} .
\end{array}\right.
$$

Integrating (3.16) in $x$ over $\mathbb{R}_{+}$leads to

$$
\frac{d}{d t} \int \mathcal{E}_{1}+\mathcal{E}_{2} d x-G_{1}(t, 0)+\int n(\bar{\psi}-\psi)^{2}+\mu \psi_{x}^{2}+n \bar{\psi}_{x}^{2} d x+\int R_{1} d x+\int R_{2} d x=-\int R_{3} d x .
$$

Under the condition (3.7), we get

$$
-G_{1}(t, 0)=-u_{-}\left[\Phi_{1}(\rho(t, 0), \widetilde{\rho}(0))+\Phi_{2}(n(t, 0), \widetilde{n}(0))\right] \geq c\left(\phi^{2}(t, 0)+\bar{\phi}^{2}(t, 0)\right) .
$$

For the case $M_{+} \neq 1$, with the help of (1.11), (3.10) and (3.11), we can obtain

$$
\int_{0}^{\infty}\left|R_{1}\right|+\left|R_{2}\right|+\left|R_{3}\right| d x \leq C \delta\left\|\left(\phi_{x}, \psi_{x}, \bar{\phi}_{x}, \bar{\psi}_{x}, \bar{\psi}-\psi\right)\right\|^{2}+C \delta\left(\phi^{2}(t, 0)+\bar{\phi}^{2}(t, 0)\right) .
$$

For the case $M_{+}=1$ and the restriction $\left|p_{1}^{\prime}\left(\rho_{+}\right)-p_{2}^{\prime}\left(n_{+}\right)\right| \leq \sqrt{2}\left|u_{+}\right| \min \left\{\left(1+\frac{\rho_{+}}{n_{+}}\right)\left[(\gamma-1) p_{1}^{\prime}\left(\rho_{+}\right)\right]^{\frac{1}{2}},(1+\right.$ $\left.\left.\frac{n_{+}}{\rho_{+}}\right)\left[(\alpha-1) p_{2}^{\prime}\left(n_{+}\right)\right]^{\frac{1}{2}}\right\}$, using (1.12)-(1.14), (3.10), (3.12), we can get

$$
\begin{aligned}
& \int R_{1}+R_{2}+R_{3} d x \\
\geq & \int(\psi, \phi) \boldsymbol{M}_{\mathbf{1}}(\psi, \phi)^{\mathrm{T}} \widetilde{u}_{x}+(\bar{\psi}, \bar{\phi}) \boldsymbol{M}_{\mathbf{2}}(\bar{\psi}, \bar{\phi})^{\mathrm{T}} \widetilde{v}_{x} d x-C \int \frac{\delta^{3}}{(1+\delta x)^{3}}\left(\phi^{2}+\psi^{2}+\bar{\phi}^{2}+\bar{\psi}^{2}\right) d x \\
& -C \delta \int|\bar{\psi}-\psi|^{2}+\psi_{x}^{2} d x-C \delta^{\frac{1}{2}}\|(\phi, \psi, \bar{\phi}, \bar{\psi})\|\left[\phi^{2}(t, 0)+\bar{\phi}^{2}(t, 0)+\left\|\left(\phi_{x}, \psi_{x}, \bar{\phi}_{x}, \bar{\phi}_{x}\right)\right\|^{2}\right]
\end{aligned}
$$




$$
\begin{aligned}
\geq & \int(\psi, \phi) \boldsymbol{M}_{\mathbf{1}}(\psi, \phi)^{\mathrm{T}} \widetilde{u}_{x}+(\bar{\psi}, \bar{\phi}) \boldsymbol{M}_{\mathbf{2}}(\bar{\psi}, \bar{\phi})^{\mathrm{T}} \widetilde{v}_{x} d x-C(\delta+\varepsilon)\left\|\left(\phi_{x}, \psi_{x}, \bar{\phi}_{x}, \bar{\psi}_{x}, \bar{\psi}-\psi\right)\right\|^{2} \\
& -C \delta\left(\phi^{2}(t, 0)+\bar{\phi}^{2}(t, 0)\right) \\
\geq & -C(\delta+\varepsilon)\left\|\left(\phi_{x}, \psi_{x}, \bar{\phi}_{x}, \bar{\psi}_{x}, \bar{\psi}-\psi\right)\right\|^{2}-C \delta\left(\phi^{2}(t, 0)+\bar{\phi}^{2}(t, 0)\right),
\end{aligned}
$$

where $\boldsymbol{M}_{1}, \boldsymbol{M}_{\mathbf{2}}$ are non-negative definite matrices defined by

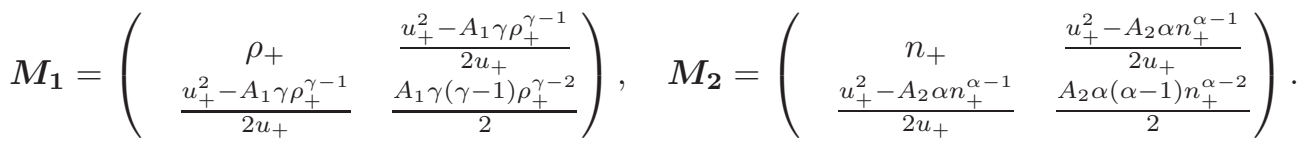

Finally, with the help of (3.18)-(3.21), we get

$$
\begin{aligned}
& \|(\phi, \psi, \bar{\phi}, \bar{\psi})\|^{2}+\int_{0}^{t}\left\|\left(\psi_{x}, \bar{\psi}_{x}, \bar{\psi}-\psi\right)\right\|^{2} d \tau+\int_{0}^{t} \phi^{2}(t, 0)+\bar{\phi}^{2}(t, 0) d \tau \\
\leq & C\left\|\left(\phi_{0}, \psi_{0}, \bar{\phi}_{0}, \bar{\psi}_{0}\right)\right\|^{2}+C \delta \int_{0}^{t}\left\|\left(\phi_{x}, \bar{\phi}_{x}\right)\right\|^{2} d \tau .
\end{aligned}
$$

Hence, we complete the proof of Lemma 3.3.

In order to complete the proof of Proposition 3.1, we need to obtain the estimates of $\left(\phi_{x}, \psi_{x}, \bar{\phi}_{x}, \bar{\psi}_{x}\right)$.

Lemma 3.4. Under the same conditions in Proposition 3.1, then the solution $(\phi, \psi, \bar{\phi}, \bar{\psi})$ to the problem (3.3)-(3.7) satisfies for $t \in[0, T]$ that

$$
\begin{aligned}
& \left\|\left(\phi_{x}, \bar{\phi}_{x}\right)\right\|^{2}+\int_{0}^{t}\left\|\left(\phi_{x}, \bar{\phi}_{x}\right)\right\|^{2} d \tau+\int_{0}^{t} \phi_{x}^{2}(t, 0)+\bar{\phi}_{x}^{2}(t, 0) d \tau \\
\leq & C\left\|\left(\phi_{0}, \psi_{0}, \phi_{0 x}, \bar{\phi}_{0}, \bar{\psi}_{0}, \bar{\phi}_{0 x}\right)\right\|^{2}+C(\varepsilon+\delta) \int_{0}^{t}\left\|\left(\psi_{x x}, \bar{\psi}_{x x}\right)\right\|^{2} .
\end{aligned}
$$

Proof. Differentiating $(3.3)_{1}$ in $x$, then multiplying the resulted equation by $\mu \phi_{x},(3.3)_{2}$ by $\widetilde{\rho}^{2} \phi_{x}$ respectively, we gain

$$
\begin{aligned}
& \left(\mu \frac{\phi_{x}^{2}}{2}\right)_{t}+\left(\mu u \frac{\phi_{x}^{2}}{2}\right)_{x}+\mu \widetilde{\rho} \phi_{x} \psi_{x x}=-\mu\left[\frac{3}{2} \psi_{x} \phi_{x}^{2}+\left(\phi \psi_{x x}+\frac{1}{2} \phi_{x} \widetilde{u}_{x}+\psi_{x} \widetilde{\rho}_{x}\right) \phi_{x}+\left(\phi \widetilde{u}_{x}+\psi \widetilde{\rho}_{x}\right)_{x} \phi_{x}\right], \\
& \left(\widetilde{\rho}^{2} \phi_{x} \psi\right)_{t}-\left(\widetilde{\rho}^{2} \phi_{t} \psi\right)_{x}+2 \widetilde{\rho} \widetilde{\rho}_{x} \phi_{t} \psi+\widetilde{\rho}^{2} \phi_{t} \psi_{x}+\widetilde{\rho}^{2} u \phi_{x} \psi_{x}+\widetilde{\rho}^{2} \frac{p_{1}^{\prime}(\rho)}{\rho} \phi_{x}^{2}-\mu \widetilde{\rho} \phi_{x} \psi_{x x}-\widetilde{\rho}^{2} \frac{n}{\rho}(\bar{\psi}-\psi) \phi_{x} \\
& =\mu \widetilde{\rho}^{2}\left(\frac{1}{\rho}-\frac{1}{\widetilde{\rho}}\right) \phi_{x} \psi_{x x}+F_{1} \widetilde{\rho}^{2} \phi_{x} .
\end{aligned}
$$

Similarly, differentiating $(3.3)_{3}$ in $x$, then multiplying the resulted equation by $\bar{\phi}_{x},(3.3)_{4}$ by $\widetilde{n} \bar{\phi}_{x}$ respectively lead to

$$
\begin{aligned}
& \left(\frac{\bar{\phi}_{x}^{2}}{2}\right)_{t}+\left(v \frac{\bar{\phi}_{x}^{2}}{2}\right)_{x}+\widetilde{n} \bar{\phi}_{x} \bar{\psi}_{x x}=-\left[\frac{3}{2} \bar{\psi}_{x} \bar{\phi}_{x}^{2}+\left(\bar{\phi} \bar{\psi}_{x x}+\frac{1}{2} \bar{\phi}_{x} \widetilde{v}_{x}+\bar{\psi}_{x} \widetilde{n}_{x}\right) \bar{\phi}_{x}-\left(\bar{\phi} \widetilde{v}_{x}+\bar{\psi} \widetilde{n}_{x}\right)_{x} \bar{\phi}_{x}\right], \\
& \left(\widetilde{n} \bar{\phi}_{x} \bar{\psi}\right)_{t}-\left(\widetilde{n} \bar{\phi}_{t} \bar{\psi}\right)_{x}+\widetilde{n}_{x} \bar{\phi}_{t} \bar{\psi}+\widetilde{n} \bar{\phi}_{t} \bar{\psi}_{x}+\widetilde{n} u \bar{\phi}_{x} \bar{\psi}_{x}+\widetilde{n} \frac{p_{2}^{\prime}(n)}{n} \bar{\phi}_{x}^{2}-\widetilde{n} \bar{\phi}_{x} \bar{\psi}_{x x}+\widetilde{n}(\bar{\psi}-\psi) \bar{\phi}_{x} \\
= & \widetilde{n} \frac{\left(\bar{\phi} \bar{\psi}_{x}\right)_{x}}{n} \bar{\phi}_{x}+\widetilde{n}\left(\frac{1}{n}-\frac{1}{\widetilde{n}}\right)\left(\widetilde{n} \bar{\psi}_{x}\right)_{x} \bar{\phi}_{x}+\widetilde{n}_{x} \bar{\phi}_{x} \bar{\psi}_{x x}+F_{2} \widetilde{n} \bar{\phi}_{x} .
\end{aligned}
$$


Adding (3.25)-(3.28) together and integrating over $\mathbb{R}_{+}$yield

$$
\begin{aligned}
& \frac{d}{d t} \int\left(\mu \frac{\phi_{x}^{2}}{2}+\frac{\bar{\phi}_{x}^{2}}{2}+\widetilde{\rho}^{2} \phi_{x} \psi+\widetilde{n} \bar{\phi}_{x} \bar{\psi}\right) d x+\int\left(\mu u \frac{\phi_{x}^{2}}{2}+v \frac{\bar{\phi}_{x}^{2}}{2}-\widetilde{\rho}^{2} \phi_{t} \psi-\widetilde{n} \bar{\phi}_{t} \bar{\psi}\right)_{x} d x \\
& +\int\left(\widetilde{\rho}^{2} \frac{p_{1}^{\prime}(\rho)}{\rho} \phi_{x}^{2}+\widetilde{n} \frac{p_{2}^{\prime}(n)}{n} \bar{\phi}_{x}^{2}\right) d x=\sum_{i=1}^{5} J_{i}
\end{aligned}
$$

where

$$
\begin{aligned}
& J_{1}=-\int\left[\widetilde{\rho}^{2}\left(\phi_{t}+u \phi_{x}\right) \psi_{x}+\widetilde{n}\left(\bar{\phi}_{t}+v \bar{\phi}_{x}\right) \bar{\psi}_{x}+2 \widetilde{\rho} \widetilde{\rho}_{x} \phi_{t} \psi+\widetilde{n}_{x} \bar{\phi}_{t} \bar{\psi}\right] d x, \\
& J_{2}=\int-\mu \phi \phi_{x} \psi_{x x}-\bar{\phi} \bar{\phi}_{x} \bar{\psi}_{x x}+\mu \widetilde{\rho}^{2}\left(\frac{1}{\rho}-\frac{1}{\widetilde{\rho}}\right) \phi_{x} \psi_{x x}+\widetilde{n}\left(\frac{1}{n}-\frac{1}{\widetilde{n}}\right)\left(\widetilde{n} \bar{\psi}_{x}\right)_{x} \bar{\phi}_{x}+\widetilde{\rho}^{2} \frac{n}{\rho}(\bar{\psi}-\psi) \phi_{x}-\widetilde{n}(\bar{\psi}-\psi) \bar{\phi}_{x} d x, \\
& J_{3}=-\int \frac{3}{2} \mu \psi_{x} \frac{\phi_{x}^{2}}{2}+\frac{3}{2} \bar{\psi}_{x} \bar{\phi}_{x}^{2} d x+\int \widetilde{n} \frac{\left(\bar{\phi} \bar{\psi}_{x}\right)_{x}}{n} \bar{\phi}_{x} d x, \quad J_{4}=\int F_{1} \widetilde{\rho}^{2} \phi_{x}+F_{2} \widetilde{n} \bar{\phi}_{x} d x, \\
& J_{5}=-\int\left[\mu\left(\frac{1}{2} \phi_{x} \widetilde{u}_{x}+\psi_{x} \widetilde{\rho}_{x}\right) \phi_{x}+\mu\left(\phi \widetilde{u}_{x}+\psi \widetilde{\rho}_{x}\right)_{x} \phi_{x}\right. \\
&\left.+\left(\frac{1}{2} \bar{\phi}_{x} \widetilde{v}_{x}+\bar{\psi}_{x} \widetilde{n}_{x}\right) \bar{\phi}_{x}-\left(\bar{\phi} \widetilde{v}_{x}+\bar{\psi} \widetilde{n}_{x}\right)_{x} \bar{\phi}_{x}-\widetilde{n}_{x} \bar{\phi}_{x} \bar{\psi}_{x x}\right] d x .
\end{aligned}
$$

First, we estimate terms in the left side of (3.29). Under the condition (3.7), the second term in the left side is estimated as follows

$$
\int\left(\mu u \frac{\phi_{x}^{2}}{2}+v \frac{\bar{\phi}_{x}^{2}}{2}-\widetilde{\rho}^{2} \phi_{t} \psi-\widetilde{n} \bar{\phi}_{t} \bar{\psi}\right)_{x} d x=-u_{-} \frac{\mu \phi_{x}^{2}(0, t)+\bar{\phi}_{x}^{2}(0, t)}{2} \geq 0,
$$

The third term is estimated as follows

$$
\int \widetilde{\rho}^{2} \frac{p_{1}^{\prime}(\rho)}{\rho} \phi_{x}^{2}+\widetilde{n} \frac{p_{2}^{\prime}(n)}{n} \bar{\phi}_{x}^{2} d x \geq c\left\|\left(\phi_{x}, \bar{\phi}_{x}\right)\right\|^{2}-C(\varepsilon+\delta)\left\|\left(\phi_{x}, \bar{\phi}_{x}\right)\right\|^{2} .
$$

We turn to estimate terms in the right hand side of (3.29). By (1.11)-(1.12), (3.3) $)_{1},(3.3)_{3},(3.11)-(3.12)$, Cauchy-Schwartz inequality and Young inequality, we can obtain

$$
\begin{gathered}
\left|J_{1}\right| \leq C\left\|\left(\psi_{x}, \bar{\psi}_{x}\right)\right\|^{2}+C \delta\left\|\left(\phi_{x}, \bar{\phi}_{x}\right)\right\|^{2}+C \delta\left(\phi^{2}(t, 0)+\bar{\phi}^{2}(t, 0)\right), \\
\left|J_{2}\right| \leq C\|(\phi, \bar{\phi})\|_{L^{\infty}}\left\|\left(\phi_{x}, \bar{\phi}_{x}, \psi_{x x}, \bar{\psi}_{x x}\right)\right\|^{2}+C_{\eta}\|\bar{\psi}-\psi\|^{2}+\eta\left\|\left(\phi_{x}, \bar{\phi}_{x}\right)\right\|^{2}+C \delta\left\|\left(\bar{\phi}_{x}, \bar{\psi}_{x}\right)\right\|^{2} \\
\leq C(\varepsilon+\delta+\eta)\left\|\left(\phi_{x}, \bar{\phi}_{x}\right)\right\|^{2}+C \varepsilon\left\|\left(\psi_{x x}, \bar{\psi}_{x x}\right)\right\|^{2}+C_{\eta}\|\bar{\psi}-\psi\|^{2}+C \delta\left\|\bar{\psi}_{x}\right\|^{2}, \\
\left|J_{3}\right| \leq C\left\|\left(\psi_{x}, \bar{\psi}_{x}\right)\right\|_{L^{\infty}}\left\|\left(\phi_{x}, \bar{\phi}_{x}\right)\right\|^{2}+C\|\bar{\phi}\|_{L^{\infty}}\left\|\left(\bar{\phi}_{x}, \bar{\psi}_{x x}\right)\right\|^{2} \\
\leq C \varepsilon\left\|\left(\phi_{x}, \psi_{x}, \bar{\phi}_{x}, \bar{\psi}_{x}\right)\right\|^{2}+C \varepsilon\left\|\left(\psi_{x x}, \bar{\psi}_{x x}\right)\right\|^{2}, \\
\left|J_{4}+J_{5}\right| \leq C \delta\left\|\left(\phi_{x}, \psi_{x}, \bar{\phi}_{x}, \bar{\psi}_{x}\right)\right\|^{2}+C \delta\left\|\bar{\psi}_{x x}\right\|^{2}+C \delta\left(\phi^{2}(t, 0)+\bar{\phi}^{2}(t, 0)\right) .
\end{gathered}
$$

Finally, the substitution of (3.30)-(3.35) into (3.29) for $\delta, \varepsilon$ and $\eta$ small enough leads to

$$
\begin{aligned}
& \frac{d}{d t} \int\left(\phi_{x}^{2}+\bar{\phi}_{x}^{2}+\widetilde{\rho}^{2} \phi_{x} \psi+\widetilde{n} \bar{\phi}_{x} \bar{\psi}\right) d x+\left\|\left(\phi_{x}, \bar{\phi}_{x}\right)\right\|^{2}+\phi_{x}^{2}(t, 0)+\bar{\phi}_{x}^{2}(t, 0) \\
\leq & C\left\|\left(\psi_{x}, \bar{\psi}_{x}, \bar{\psi}-\psi_{x}\right)\right\|^{2}+C(\delta+\varepsilon)\left\|\left(\psi_{x x}, \bar{\psi}_{x x}\right)\right\|^{2}+C \delta\left(\phi^{2}(t, 0)+\bar{\phi}^{2}(t, 0)\right) .
\end{aligned}
$$


Integrating (3.36) over $[0, t]$, we can obtain

$$
\left\|\left(\phi_{x}, \bar{\phi}_{x}\right)\right\|^{2}+\int_{0}^{t}\left\|\left(\phi_{x}, \bar{\phi}_{x}\right)\right\|^{2} d \tau \leq C\left\|\left(\phi_{0}, \bar{\phi}_{0}, \psi_{0}, \bar{\psi}_{0}, \phi_{0 x}, \bar{\phi}_{0 x}\right)\right\|^{2}+C(\delta+\varepsilon) \int_{0}^{t}\left\|\left(\psi_{x x}, \bar{\psi}_{x x}\right)\right\|^{2} d \tau,
$$

where we have used (3.13) and Young inequality. Hence, we complete the proof of Lemma 3.4.

Lemma 3.5. Under the same conditions in Proposition 3.1, then the solution $(\phi, \psi, \bar{\phi}, \bar{\psi})$ to the problem (3.3)-(3.7) satisfies for $t \in[0, T]$ that

$$
\left\|\left(\psi_{x}, \bar{\psi}_{x}\right)\right\|^{2}+\int_{0}^{t}\left\|\left(\psi_{x x}, \bar{\psi}_{x x}\right)\right\|^{2} d \tau \leq C\left\|\left(\phi_{0}, \psi_{0}, \bar{\phi}_{0}, \bar{\psi}_{0}\right)\right\|_{1}^{2} .
$$

Proof. Multiplying $(3.3)_{2}$ by $-\psi_{x x},(3.3)_{4}$ by $-\bar{\psi}_{x x}$, respectively, then adding them together and integrating the resulted equation in $x$ over $\mathbb{R}_{+}$imply

$$
\frac{d}{d t} \int \frac{\psi_{x}^{2}}{2}+\frac{\bar{\psi}_{x}^{2}}{2} d x+\int \mu \frac{1}{\rho} \psi_{x x}^{2}+\bar{\psi}_{x x}^{2} d x=\sum_{i=1}^{3} K_{i},
$$

where

$$
\begin{aligned}
K_{1}= & \int\left[u \psi_{x} \psi_{x x}-\frac{n}{\rho}(\bar{\psi}-\psi) \psi_{x x}+\frac{p_{1}^{\prime}(\rho)}{\rho} \phi_{x} \psi_{x x}+v \bar{\psi}_{x} \bar{\psi}_{x x}+(\bar{\psi}-\psi) \bar{\psi}_{x x}+\frac{p_{2}^{\prime}(n)}{n} \bar{\phi}_{x} \bar{\psi}_{x x}\right] d x, \\
K_{2}= & \int\left[\widetilde{u}_{x} \psi \psi_{x x}-\mu \widetilde{u}_{x x}\left(\frac{1}{\rho}-\frac{1}{\widetilde{\rho}}\right) \psi_{x x}+\left(\frac{p_{1}^{\prime}(\rho)}{\rho}-\frac{p_{1}^{\prime}(\widetilde{\rho})}{\widetilde{\rho}}\right) \psi_{x x} \widetilde{\rho}_{x}-\left(\frac{n}{\rho}-\frac{\widetilde{n}}{\widetilde{\rho}}\right)(\widetilde{v}-\widetilde{u}) \psi_{x x}+\widetilde{v}_{x} \bar{\psi} \bar{\psi}_{x x}\right. \\
& \left.+\left(\widetilde{n} \widetilde{v}_{x}\right)_{x}\left(\frac{1}{n}-\frac{1}{\widetilde{n}}\right) \bar{\psi}_{x x}+\left(\frac{p_{2}^{\prime}(n)}{n}-\frac{p_{2}^{\prime}(\widetilde{n})}{\widetilde{n}}\right) \bar{\psi}_{x x} \widetilde{n}_{x}-\frac{\widetilde{n}_{x}}{\widetilde{n}} \bar{\psi}_{x} \bar{\psi}_{x x}-\frac{\left(\bar{\phi} \widetilde{v}_{x}\right)_{x}}{n} \bar{\psi}_{x x}\right] d x, \\
K_{3}= & -\int\left[\frac{\left(\bar{\phi} \bar{\psi}_{x}\right)_{x}}{n} \bar{\psi}_{x x}+\left(\frac{1}{n}-\frac{1}{\widetilde{n}}\right)\left(\widetilde{n} \bar{\psi}_{x}\right)_{x} \bar{\psi}_{x x}\right] d x .
\end{aligned}
$$

We estimate terms in the left side of (3.39). By the decomposition $\frac{1}{\rho}=\left(\frac{1}{\rho}-\frac{1}{\widetilde{\rho}}\right)+\left(\frac{1}{\widetilde{\rho}}-\frac{1}{\rho_{+}}\right)+\frac{1}{\rho_{+}}$, the second term is estimated as follows:

$$
\begin{aligned}
\int \frac{\mu}{\rho} \psi_{x x}^{2}+\bar{\psi}_{x x}^{2} d x & \geq \frac{\mu}{\rho_{+}}\left\|\psi_{x x}\right\|^{2}+\left\|\bar{\psi}_{x x}\right\|^{2}-C\left(\|\phi\|_{L^{\infty}}+\delta\right)\left\|\psi_{x x}\right\|^{2} \\
& \geq \frac{\mu}{\rho_{+}}\left\|\psi_{x x}\right\|^{2}+\left\|\bar{\psi}_{x x}\right\|^{2}-C(\varepsilon+\delta)\left\|\psi_{x x}\right\|^{2} .
\end{aligned}
$$

We turn to estimate terms in the right side of (3.39). With the aid of (1.11), Sobolev inequality and Cauchy-Schwarz inequality, we have

$$
\begin{gathered}
\left|K_{1}\right| \leq \frac{\mu}{16 \rho_{+}}\left\|\psi_{x x}\right\|^{2}+\frac{1}{16}\left\|\bar{\psi}_{x x}\right\|^{2}+C\left\|\left(\phi_{x}, \psi_{x}, \bar{\phi}_{x}, \bar{\psi}_{x}, \bar{\psi}-\psi\right)\right\|^{2}, \\
\left|K_{2}\right| \leq C \delta\left\|\left(\phi_{x}, \psi_{x}, \bar{\phi}_{x}, \bar{\psi}_{x}\right)\right\|^{2}+C \delta\left\|\left(\psi_{x x}, \bar{\psi}_{x x}\right)\right\|^{2}+C \delta\left(\phi^{2}(t, 0)+\bar{\phi}^{2}(t, 0)\right), \\
\left|K_{3}\right| \leq C\|\bar{\phi}\|_{L^{\infty}}\left\|\left(\bar{\psi}_{x}, \bar{\psi}_{x x}\right)\right\|^{2}+C\left\|\bar{\psi}_{x}\right\|_{L^{\infty}}\left\|\bar{\psi}_{x x}\right\|\left\|\bar{\phi}_{x}\right\| \leq C \varepsilon\left\|\left(\bar{\psi}_{x}, \bar{\psi}_{x x}\right)\right\|^{2} .
\end{gathered}
$$

Finally, taking $\delta$ and $\varepsilon$ small enough and substituting of (3.40)-(3.43) into (3.39), we obtain

$$
\frac{d}{d t} \int \psi_{x}^{2}+\bar{\psi}_{x}^{2} d x+\frac{\mu}{2 \rho_{+}}\left\|\psi_{x x}\right\|^{2}+\frac{1}{2}\left\|\bar{\psi}_{x x}\right\|^{2} \leq C\left\|\left(\phi_{x}, \psi_{x}, \bar{\phi}_{x}, \bar{\psi}_{x}, \bar{\psi}-\psi\right)\right\|^{2}+C \delta\left(\phi^{2}(t, 0)+\bar{\phi}^{2}(t, 0)\right) .
$$


Integrating (3.44) in $\tau$ over $[0, t]$ leads to

$$
\|\left(\psi_{x}, \bar{\psi}_{x}\left\|^{2}+\int_{0}^{t}\right\|\left(\psi_{x x}, \bar{\psi}_{x x}\right)\left\|^{2} d \tau \leq C\right\|\left(\phi_{0}, \psi_{0}, \bar{\phi}_{0}, \bar{\psi}_{0}\right) \|_{1}^{2}\right.
$$

where we have used (3.13), (3.24) and the smallness of $\delta$ and $\varepsilon$. Thus, we complete the proof of Lemma 3.5 .

With the help of Lemmas 3.3-3.5, we get (3.9) and complete the proof of Proposition 3.1.

\section{Time convergence rates}

\subsection{Convergence rate of supersonic steady-state}

Proposition 4.1. Assume that the same conditions in Theorem 1.3 for $M_{+}>1$ hold and let $(\phi, \psi, \bar{\phi}, \bar{\psi})$ be a solution to the IBVP (3.3)-(3.7) satisfying $(1+x)^{\frac{\nu}{2}}(\phi, \psi, \bar{\phi}, \bar{\psi}) \in C\left([0, T] ; L^{2}\right)$ for any time $T>0$. Then for $\nu \in[0, \lambda]$, there exist positive constants $\varepsilon>0$ and $C>0$ independent of $T$ such that if

$$
\sup _{0 \leq t \leq T}\|(\phi, \psi, \bar{\phi}, \bar{\psi})\|_{1}+\delta \leq \varepsilon
$$

is satisfied, it holds for arbitrary $t \in[0, T]$ that

$$
\begin{aligned}
& (1+t)^{\lambda-\nu+\theta}\left(\|(\phi, \psi, \bar{\phi}, \bar{\psi})\|_{1}+\|(\phi, \psi, \bar{\phi}, \bar{\psi})\|_{a, \nu}^{2}\right)+\nu \int_{0}^{t}(1+\tau)^{\lambda-\nu+\theta}\|(\phi, \bar{\phi}, \psi, \bar{\psi})\|_{a, \nu-1}^{2} d \tau \\
& +\int_{0}^{t}(1+\tau)^{\lambda-\nu+\theta}\left\|\left(\psi_{x}, \bar{\psi}_{x}, \bar{\psi}-\psi\right)\right\|_{a, \nu}^{2} d \tau+\int_{0}^{t}(1+\tau)^{\lambda-\nu+\theta}\left\|\left(\phi_{x}, \psi_{x x}, \bar{\phi}_{x}, \bar{\psi}_{x x}\right)\right\|_{1}^{2} d \tau \\
\leq & C(1+t)^{\theta}\left(\left\|\left(\phi_{0}, \psi_{0}, \bar{\phi}_{0}, \bar{\psi}_{0}\right)\right\|_{1}^{2}+\left\|\left(\phi_{0}, \psi_{0}, \bar{\phi}_{0}, \bar{\psi}_{0}\right)\right\|_{a, \lambda}^{2}\right),
\end{aligned}
$$

with $\theta>0$.

Our first goal is to obtain the basic weighted energy estimates of $(\phi, \psi, \bar{\phi}, \bar{\psi})$.

Lemma 4.2. Under the same conditions in Proposition 4.1, then the solution $(\phi, \psi, \bar{\phi}, \bar{\psi})$ to the IBVP (3.3)-(3.7) satisfies for $t \in[0, T]$ that

$$
\begin{aligned}
& (1+t)^{\xi}\|(\phi, \psi, \bar{\phi}, \bar{\psi})\|_{a, \nu}^{2}+\nu \int_{0}^{t}(1+\tau)^{\xi}\|(\phi, \psi, \bar{\phi}, \bar{\psi})\|_{a, \nu-1}^{2} d \tau \\
& +\int_{0}^{t}(1+\tau)^{\xi}\left\|\left(\psi_{x}, \bar{\psi}_{x}, \bar{\psi}-\psi\right)\right\|_{a, \nu}^{2} d \tau+\int_{0}^{t}(1+\tau)^{\xi}\left(\phi^{2}(t, 0)+\bar{\phi}^{2}(t, 0)\right) d \tau \\
\leq & C\left\|\left(\phi_{0}, \psi_{0}, \bar{\phi}_{0}, \bar{\psi}_{0}\right)\right\|_{a, \lambda}^{2}+C \delta \int_{0}^{t}(1+\tau)^{\xi}\left\|\left(\phi_{x}, \bar{\phi}_{x}\right)\right\|^{2} d \tau \\
& +C \nu \int_{0}^{t}(1+\tau)^{\xi}\left\|\left(\psi_{x}, \bar{\psi}_{x}, \bar{\psi}-\psi\right)\right\|_{a, \nu-1}^{2} d \tau+C \xi \int_{0}^{t}(1+\tau)^{\xi-1}\|(\phi, \psi, \bar{\phi}, \bar{\psi})\|_{a, \nu}^{2} d \tau .
\end{aligned}
$$

with $\xi \geq 0$. 
Proof. We multiply (3.16) by $W_{a, \nu}$, where $W_{a, \nu}:=(1+x)^{\nu}$ is a space weight function. We integrate the resulted equality over $\mathbb{R}_{+}$to obtain

$$
\begin{aligned}
& \frac{d}{d t} \int W_{a, \nu}\left(\mathcal{E}_{1}+\mathcal{E}_{2}\right)-\left(W_{a, \nu} G_{1}\right)(t, 0)-\int W_{a, \nu-1} G_{1} d x-\int W_{a, \nu-1} G_{2} d x \\
& +\int W_{a, \nu}\left[\mu \psi_{x}^{2}+n \bar{\psi}_{x}^{2}+n(\bar{\psi}-\psi)^{2}\right] d x \\
& =-\int W_{a, \nu}\left(R_{1}+R_{2}+R_{3}\right) d x,
\end{aligned}
$$

where $G_{i}$ for $i=1,2, R_{j}$ for $j=1,2,3$ and $\mathcal{E}_{k}, k=1,2$ are defined by (3.17).

First, we estimate terms on the left hand side of (4.4). Under the condition (3.7), the second term on the left hand side is estimated as

$$
-\left(W_{a, \nu} G_{1}\right)(t, 0)=\left|u_{-}\right|\left[\Phi_{1}(\rho(t, 0), \widetilde{\rho}(0))+\Phi_{2}(n(t, 0), \widetilde{n}(0))\right] \geq c\left(\phi^{2}(t, 0)+\bar{\phi}^{2}(t, 0)\right),
$$

We decompose $\psi$ as $\psi=\bar{\psi}+(\psi-\bar{\psi})$ and use (1.11) to gain

$$
\begin{aligned}
& -\nu \int W_{a, \nu-1} G_{1} d x \\
\geq & \nu \int W_{a, \nu-1}\left[-\frac{u_{+}}{2} n_{+} \bar{\psi}^{2}-\frac{u_{+}}{2} A_{1} \gamma \rho_{+}^{\gamma-2} \phi^{2}-A_{1} \gamma \rho_{+}^{\gamma-1} \phi \bar{\psi}-\frac{u_{+}}{2} \rho_{+} \bar{\psi}^{2}-A_{2} \alpha n_{+}^{\alpha-1} \bar{\phi} \bar{\psi}\right. \\
& \left.-\frac{u_{+}}{2} A_{2} \alpha n_{+}^{\alpha-2} \bar{\phi}^{2}-\frac{u_{+}}{2} \rho_{+}|\psi-\bar{\psi}|^{2}-\rho_{+} u_{+} \bar{\psi}(\psi-\bar{\psi})-A_{1} \gamma \rho_{+}^{\gamma-1} \phi(\psi-\bar{\psi})\right] d x \\
& -C(\delta+\varepsilon)\|(\phi, \psi, \bar{\phi}, \bar{\psi})\|_{a, \nu-1}^{2} \\
\geq & \nu \int W_{a, \nu-1}\left[\frac{1}{2}(\phi, \bar{\phi}, \bar{\psi}) M_{3}(\phi, \bar{\phi}, \bar{\psi})^{\mathrm{T}}-\rho_{+} u_{+} \bar{\psi}(\psi-\bar{\psi})-A_{1} \gamma \rho_{+}^{\gamma-1} \phi(\psi-\bar{\psi})\right] d x \\
& -C(\delta+\varepsilon)\|(\phi, \psi, \bar{\phi}, \bar{\psi})\|_{a, \nu-1}^{2},
\end{aligned}
$$

where the symmetric matrix $\boldsymbol{M}_{\mathbf{3}}$ is denoted by

$$
\boldsymbol{M}_{\mathbf{3}}=\left(\begin{array}{ccc}
-A_{1} \gamma \rho_{+}^{\gamma-2} u_{+} & 0 & -A_{1} \gamma \rho_{+}^{\gamma-1} \\
0 & -A_{2} \alpha n_{+}^{\alpha-2} u_{+} & -A_{2} \alpha n_{+}^{\alpha-1} \\
-A_{1} \gamma \rho_{+}^{\gamma-1} & -A_{2} \alpha n_{+}^{\alpha-1} & -\left(\rho_{+}+n_{+}\right) u_{+}
\end{array}\right) .
$$

It is easy to verify that $M_{3}$ is a positive definite matrix under the condition $M_{+}>1$. Hence, the estimate of the third term on the left hand side is obtained under the condition $\varepsilon, \delta$ and $\eta$ small enough that

$$
\begin{aligned}
& -\nu \int W_{a, \nu-1} G_{1} d x \\
\geq & c \nu\|(\phi, \psi, \bar{\phi}, \bar{\psi})\|_{a, \nu-1}^{2}-\eta \nu\|(\phi, \bar{\psi})\|_{a, \nu-1}^{2}-C_{\eta} \nu\|\bar{\psi}-\psi\|_{a, \nu-1}^{2}-C(\varepsilon+\delta)\|(\phi, \psi, \bar{\phi}, \bar{\psi})\|_{a, \nu-1}^{2} \\
\geq & c \nu\|(\phi, \psi, \bar{\phi}, \bar{\psi})\|_{a, \nu-1}^{2}-C \nu\|\bar{\psi}-\psi\|_{a, \nu-1}^{2},
\end{aligned}
$$

The forth and the fifth terms on the left hand side are estimated as below

$$
-\nu \int W_{a, \nu-1} G_{2} d x \leq \nu \eta\|(\psi, \bar{\psi})\|_{a, \nu-1}^{2}+\nu C_{\eta}\left\|\left(\psi_{x}, \bar{\psi}_{x}\right)\right\|_{a, \nu-1}^{2}+C \delta\|(\bar{\phi}, \bar{\psi})\|_{a, \nu-1}^{2}
$$




$$
\int W_{a, \nu}\left[\mu \psi_{x}^{2}+n \bar{\psi}_{x}^{2}+n(\psi-\bar{\psi})^{2}\right] d x \geq c\left\|\left(\psi_{x}, \bar{\psi}_{x}, \bar{\psi}-\psi\right)\right\|_{a, \nu}^{2}-C(\varepsilon+\delta)\left\|\left(\psi_{x}, \bar{\psi}_{x}, \psi-\bar{\psi}\right)\right\|_{a, \nu}^{2}
$$

With the help of (1.11), (3.11), Sobolev inequality and Cauchy-Schwarz inequality, we have the estimate of the term on the right hand side of (4.4) as

$$
\begin{aligned}
\left|\int W_{a, \nu}\left(R_{1}+R_{2}+R_{3}\right) d x\right| & \leq C \delta \int e^{-\frac{c_{0}}{2} x}\left(\phi^{2}+\psi^{2}+\bar{\phi}^{2}+\bar{\psi}^{2}+|\bar{\psi}-\psi|^{2}\right) d x \\
& \leq C \delta\left\|\left(\phi_{x}, \psi_{x}, \bar{\phi}_{x}, \bar{\psi}_{x}, \bar{\psi}-\psi\right)\right\|^{2}+C \delta\left(\phi^{2}(t, 0)+\bar{\phi}^{2}(t, 0)\right) .
\end{aligned}
$$

Finally, with $\eta, \delta$ and $\varepsilon$ suitably small, the substitution of (4.5)-(4.11) into (4.4) leads to

$$
\begin{aligned}
& \frac{d}{d t} \int W_{a, \nu}\left(\mathcal{E}_{1}+\mathcal{E}_{2}\right) d x+c \nu\|(\phi, \psi, \bar{\phi}, \bar{\psi})\|_{a, \nu-1}^{2}+c\left\|\left(\psi_{x}, \bar{\psi}_{x}, \psi-\bar{\psi}\right)\right\|_{a, \nu}^{2}+c\left(\phi^{2}(t, 0)+\bar{\phi}^{2}(t, 0)\right) \\
\leq & C \delta\left\|\left(\phi_{x}, \bar{\phi}_{x}\right)\right\|^{2}+C \nu\left\|\left(\psi_{x}, \bar{\psi}_{x}, \psi-\bar{\psi}\right)\right\|_{a, \nu-1}^{2} .
\end{aligned}
$$

Multiplying (4.12) by $(1+\tau)^{\xi}$ and integrating the resulted equation in $\tau$ over $[0, t]$ yield for $\xi \geq 0$ that

$$
\begin{aligned}
& (1+t)^{\xi}\|(\phi, \psi, \bar{\phi}, \bar{\psi})\|_{a, \nu}^{2}+\nu \int_{0}^{t}(1+\tau)^{\xi}\|(\phi, \psi, \bar{\phi}, \bar{\psi})\|_{a, \nu-1}^{2} d \tau \\
& \quad+\int_{0}^{t}(1+\tau)^{\xi}\left\|\left(\psi_{x}, \bar{\psi}_{x}, \psi-\bar{\psi}\right)\right\|_{a, \nu}^{2} d \tau+\int_{0}^{t}(1+\tau)^{\xi}\left(\phi^{2}(t, 0)+\bar{\phi}^{2}(t, 0)\right) d \tau \\
& \leq C\left\|\left(\phi_{0}, \psi_{0}, \bar{\phi}_{0}, \bar{\psi}_{0}\right)\right\|_{a, \lambda}^{2}+C \delta \int_{0}^{t}(1+\tau)^{\xi}\left\|\left(\phi_{x}, \bar{\phi}_{x}\right)\right\|^{2} d \tau \\
& \quad+C \nu \int_{0}^{t}(1+\tau)^{\xi}\left\|\left(\psi_{x}, \bar{\psi}_{x}, \psi-\bar{\psi}\right)\right\|_{a, \nu-1}^{2} d \tau+C \xi \int_{0}^{t}(1+x)^{\xi-1}\|(\phi, \psi, \bar{\phi}, \bar{\psi})\|_{a, \nu}^{2} d \tau,
\end{aligned}
$$

Hence we obtain (4.3) and complete the proof of Lemma 4.2.

To prove Proposition 4.1, we need to obtain the following weighted energy estimates of $\left(\phi_{x}, \psi_{x}, \bar{\phi}_{x}, \bar{\psi}_{x}\right)$. By similar arguments as showing Lemmas 3.4-3.5, we get the following Lemmas. The details are omitted.

Lemma 4.3. Under the same conditions in Proposition 4.1, then the solution $(\phi, \psi, \bar{\phi}, \bar{\psi})$ to the IBVP (3.3)-(3.7) satisfies for $t \in[0, T]$ that

$$
\begin{aligned}
& (1+t)^{\xi}\left\|\left(\phi_{x}, \bar{\phi}_{x}\right)\right\|^{2}+\int_{0}^{t}(1+\tau)^{\xi}\left\|\left(\phi_{x}, \bar{\phi}_{x}\right)\right\|^{2} d \tau \\
\leq & C\left(\left\|\left(\phi_{0}, \psi_{0}, \bar{\phi}_{0}, \bar{\psi}_{0}\right)\right\|_{a, \lambda}^{2}+\left\|\left(\phi_{0 x}, \bar{\phi}_{0 x}\right)\right\|^{2}\right)+C \varepsilon \int_{0}^{t}(1+\tau)^{\xi}\left\|\left(\psi_{x x}, \bar{\psi}_{x x}\right)\right\|_{a, \nu}^{2} d \tau \\
& +C \xi \int_{0}^{t}(1+\tau)^{\xi-1}\left(\|(\phi, \psi, \bar{\phi}, \bar{\psi})\|_{a, \nu}^{2}+\left\|\left(\phi_{x}, \bar{\phi}_{x}\right)\right\|^{2}\right) d \tau,
\end{aligned}
$$

with $\xi \geq 0$.

Lemma 4.4. Under the same conditions in Proposition 4.1 hold, then the solution $(\phi, \psi, \bar{\phi}, \bar{\psi})$ to the IBVP (3.3)-(3.7) satisfies for $t \in[0, T]$ that

$$
\begin{aligned}
& (1+t)^{\xi}\left\|\left(\psi_{x}, \bar{\psi}_{x}\right)\right\|^{2}+\int_{0}^{t}(1+\tau)^{\xi}\left\|\left(\psi_{x x}, \bar{\psi}_{x x}\right)\right\|^{2} d \tau \\
\leq & C\left(\left\|\left(\phi_{0}, \psi_{0}, \bar{\phi}_{0}, \bar{\psi}_{0}\right)\right\|_{a, \lambda}^{2}+\left\|\left(\phi_{0 x}, \bar{\phi}_{0 x}, \psi_{0 x}, \bar{\psi}_{0 x}\right)\right\|^{2}\right) \\
& +C \xi \int_{0}^{t}(1+\tau)^{\xi-1}\left(\|(\phi, \psi, \bar{\phi}, \bar{\psi})\|_{a, \nu}^{2}+\left\|\left(\phi_{x}, \psi_{x}, \bar{\phi}_{x}, \bar{\psi}_{x}\right)\right\|^{2}\right) d \tau
\end{aligned}
$$


with $\xi \geq 0$.

Proof of Proposition 4.1 For $\nu \in[0, \lambda]$ and $\xi \geq 0$, with the three Lemmas 4.2-4.4 above leads to

$$
\begin{aligned}
& (1+t)^{\xi}\left(\|(\phi, \psi, \bar{\phi}, \bar{\psi})\|_{a, \nu}^{2}+\left\|\left(\phi_{x}, \psi_{x}, \bar{\psi}_{x}, \bar{\phi}_{x}\right)\right\|^{2}\right)+\nu \int(1+t)^{\xi}\|(\phi, \psi, \bar{\phi}, \bar{\psi})\|_{a, \nu-1}^{2} d \tau \\
& +\int_{0}^{t}(1+\tau)^{\xi}\left\|\left(\psi_{x}, \bar{\psi}_{x}, \psi-\bar{\psi}\right)\right\|_{a, \nu}^{2} d \tau+\int_{0}^{t}(1+\tau)^{\xi}\left\|\left(\phi_{x}, \psi_{x x}, \bar{\phi}_{x}, \bar{\psi}_{x x}\right)\right\|^{2} d \tau \\
\leq & C\left(\left\|\left(\phi_{0}, \psi_{0}, \bar{\phi}_{0}, \bar{\psi}_{0}\right)\right\|_{a, \lambda}^{2}+\left\|\left(\phi_{0 x}, \psi_{0 x}, \bar{\phi}_{0 x}, \bar{\psi}_{0 x}\right)\right\|^{2}\right)+C \nu \int_{0}^{t}(1+\tau)^{\xi}\left\|\left(\psi_{x}, \bar{\psi}_{x}, \bar{\psi}-\psi\right)\right\|_{a, \nu-1}^{2} d \tau \\
& +C \xi \int_{0}^{t}(1+\tau)^{\xi-1}\left(\|(\phi, \psi, \bar{\phi}, \bar{\psi})\|_{a, \nu}+\left\|\left(\phi_{x}, \psi_{x}, \bar{\psi}_{x}, \bar{\phi}_{x}\right)\right\|^{2}\right)
\end{aligned}
$$

where $C>0$ is a generic positive constant independent of $T, \nu$, and $\xi$. Hence, applying induction arguments similar to those used in $[2,17,24]$ to $(4.16)$ lead to the desired estimate $(4.2)$.

\subsection{Convergence rate of sonic steady-state}

The function space $Y_{W}(0, T)$ for $T>0$ is denoted by

$$
\begin{aligned}
Y_{W}(0, T):=\{(\phi, \psi, \bar{\phi}, \bar{\psi}) \mid & (\phi, \psi, \bar{\phi}, \bar{\psi}) \in C\left([0, T] ; H_{W}^{1}\right) \\
\left(\phi_{x}, \bar{\phi}_{x}\right) & \left.\in L^{2}\left([0, T] ; L_{W}^{2}\right),\left(\psi_{x}, \bar{\psi}_{x}\right) \in L^{2}\left([0, T] ; H_{W}^{1}\right)\right\} .
\end{aligned}
$$

Proposition 4.5. Assume that $1 \leq \lambda<2+\sqrt{8+\frac{1}{1+b^{2}}}$ with $b:=\frac{\rho_{+}\left(u_{+}^{2}-p_{1}^{\prime}\left(\rho_{+}\right)\right)}{\left|u_{+}\right| \sqrt{\left(\mu+n_{+}\right) n_{+}}}$and that the same conditions in Theorem 1.3 hold for $M_{+}=1$. Let $(\phi, \psi, \bar{\phi}, \bar{\psi})$ be a solution to the IBVP (3.3)-(3.7) satisfying $(\phi, \psi, \bar{\phi}, \bar{\psi}) \in Y_{\sigma^{-\frac{\lambda}{2}}}(0, T)$ for any time $T>0$. Then for arbitrary $\nu \in(0, \lambda]$, there exist positive constants $\varepsilon>0$ and $C>0$ independent of $T$ such that if

$$
\sup _{0 \leq t \leq T}\left\|\sigma^{-\frac{\lambda}{2}}(\phi, \psi, \bar{\phi}, \bar{\psi})(t)\right\|_{1}+\delta^{\frac{1}{2}} \leq \varepsilon
$$

is satisfied, it holds for arbitrary $t \in[0, T]$ that

$$
\begin{aligned}
& \quad(1+\delta t)^{\frac{\lambda-\nu}{2}+\beta}\left\|\sigma^{-\frac{\nu}{2}}(\phi, \psi, \bar{\phi}, \bar{\psi})\right\|_{1}^{2}+\int_{0}^{t}(1+\delta \tau)^{\frac{\lambda-\nu}{2}+\beta}\left\|\sigma^{-\frac{\nu-2}{2}}(\phi, \psi, \bar{\phi}, \bar{\psi})\right\|^{2} d \tau \\
& \quad+\int_{0}^{t}(1+\delta \tau)^{\frac{\lambda-\nu}{2}+\beta}\left\|\sigma^{-\frac{\nu}{2}}\left(\phi_{x}, \psi_{x}, \bar{\phi}_{x}, \bar{\psi}_{x}\right)\right\|^{2} d \tau+\int_{0}^{t}(1+\delta \tau)^{\frac{\lambda-\nu}{2}+\beta}\left\|\sigma^{-\frac{\nu}{2}}\left(\psi_{x x}, \bar{\psi}_{x x}, \bar{\psi}-\psi\right)\right\|^{2} d \tau \\
& \leq C(1+\delta t)^{\beta}\left\|\sigma^{-\frac{\lambda}{2}}\left(\phi_{0}, \psi_{0}, \phi_{0 x}, \psi_{0 x}, \bar{\phi}_{0}, \bar{\psi}_{0}, \bar{\phi}_{0 x}, \bar{\psi}_{0 x}\right)\right\|^{2},
\end{aligned}
$$

with $\beta>0$.

Under the condition $\lambda \geq 1$ and (4.18), it is easy to gain the following estimate:

$$
\left\|\sigma^{-\frac{1}{2}}(\phi, \psi, \bar{\phi}, \bar{\psi})\right\|_{L^{\infty}} \leq\left\|\sigma^{-\frac{\lambda}{2}}(\phi, \psi, \bar{\phi}, \bar{\psi})\right\|_{1} \leq \sqrt{2} \varepsilon \text {. }
$$

To deal with some nonlinear terms, we use the following inequality as in $[18,26,37]$. 
Lemma 4.6 ( [37] ). Let $\nu \geq 1$. Then a function $\phi(t, x) \in H_{\sigma^{-\frac{\nu}{2}}}^{1}\left(\mathbb{R}_{+}\right)$satisfies

$$
\int \sigma^{-\frac{\nu-1}{2}}|\phi|^{3} d x \leq C\left\|\sigma^{-\frac{1}{2}} \phi\right\|\left(\sigma(0) \phi^{2}(t, 0)+\left\|\sigma^{-\frac{\nu}{2}} \phi_{x}\right\|^{2}+\left\|\sigma^{-\frac{\nu-2}{2}} \phi\right\|^{2}\right),
$$

where the function $\sigma(x) \geq 0$ is defined by (2.28) with $\sigma(0)$ small enough.

To gain faster decay rates, it is necessary to use the following Hardy type inequality which is proved in [16].

Lemma 4.7 ( [16] ). Let $\zeta \in C^{1}[0, \infty)$ satisfies $\zeta>0, \zeta_{x}>0$ and $\zeta(x) \rightarrow \infty$ for $x \rightarrow \infty$. Then we have

$$
\int_{\mathbb{R}_{+}} \psi^{2} \zeta_{x} d x \leq 4 \int_{\mathbb{R}_{+}} \psi_{x}^{2} \frac{\zeta^{2}}{\zeta_{x}} d x
$$

for $\psi$ satisfying $\psi(t, 0)=0$ and $w \psi \in H^{1}\left(\mathbb{R}_{+}\right)$, with the function $w:=\frac{\zeta^{2}}{\zeta_{x}}$.

With the aid of Lemmas 4.6-4.7, we obtain the weighted $L^{2}$ estimate of $(\phi, \psi, \bar{\phi}, \bar{\psi})$.

Lemma 4.8. Under the same conditions in Proposition 4.5, then the solution $(\phi, \psi, \bar{\phi}, \bar{\psi})$ to the problem (3.3)-(3.7) satisfies for $t \in[0, T]$ that

$$
\begin{aligned}
& (1+\delta \tau)^{\xi}\left\|\sigma^{-\frac{\nu}{2}}(\phi, \psi, \bar{\phi}, \bar{\psi})\right\|^{2}+\int_{0}^{t}(1+\delta \tau)^{\xi}\left\|\sigma^{-\frac{\nu-2}{2}}(\phi, \psi, \bar{\phi}, \bar{\psi})\right\|^{2} d \tau \\
& +\int_{0}^{t}(1+\delta \tau)^{\xi}\left\|\sigma^{-\frac{\nu}{2}}\left(\psi_{x}, \bar{\psi}_{x}, \bar{\psi}-\psi\right)\right\|^{2} d \tau+\int_{0}^{t}(1+\delta \tau)^{\xi} \frac{1}{\delta^{\nu}}\left(\phi^{2}(t, 0)+\bar{\phi}^{2}(t, 0)\right) d \tau \\
\leq & C\left\|\sigma^{-\frac{\lambda}{2}}\left(\phi_{0}, \psi_{0}, \bar{\phi}_{0}, \bar{\psi}_{0}\right)\right\|^{2}+C \delta \int_{0}^{t}(1+\delta \tau)^{\xi}\left\|\sigma^{-\frac{\nu}{2}}\left(\phi_{x}, \bar{\phi}_{x}\right)\right\|^{2} d \tau \\
& +C \delta \xi \int_{0}^{t}(1+\delta \tau)^{\xi-1}\left\|\sigma^{-\frac{\nu}{2}}(\phi, \psi, \bar{\phi}, \bar{\psi})\right\|^{2} d \tau .
\end{aligned}
$$

with $\xi \geq 0$.

Proof. We multiply (3.16) by the space weight function $\sigma^{-\nu}$, where the space weight function $\sigma \geq 0$ satisfies (1.14) and (2.28). Then, we integrate the resulted equation over $\mathbb{R}_{+}$to get

$$
\begin{aligned}
& \frac{d}{d t} \int \sigma^{-\nu}\left(\mathcal{E}_{1}+\mathcal{E}_{2}\right) d x-\left(\sigma^{-\nu} G_{1}\right)(t, 0)-a \nu \int \sigma^{-(\nu-1)} G_{1} d x-a \nu \int \sigma^{-(\nu-1)} G_{2} d x \\
& +\int \sigma^{-\nu} n(\bar{\psi}-\psi)^{2} d x+\int \sigma^{-\nu}\left(\mu \psi_{x}^{2}+n \bar{\psi}_{x}^{2}\right) d x+\int \sigma^{-\nu} R_{1} d x \\
& =-\int \sigma^{-\nu} R_{2} d x-\int \sigma^{-\nu} R_{3} d x,
\end{aligned}
$$

where $G_{i}$ for $i=1,2, R_{j}$ for $j=1,2,3$ and $\mathcal{E}_{k}, k=1,2$ are defind by (3.17).

First, we estimate terms on the left hand side of (4.24). Under the condition (3.7), the second term on the left hand side is estimated as

$$
-\left(\sigma^{-\nu} G_{1}\right)(t, 0) \geq \frac{c}{\delta^{\nu}}\left(\phi^{2}(t, 0)+\bar{\phi}^{2}(t, 0)\right) \geq 0
$$


For the third term on the left hand side, using (4.21) and $\psi=\bar{\psi}+(\psi-\bar{\psi})$ yields

$$
\begin{aligned}
& -a \nu \int \sigma^{-(\nu-1)} G_{1} d x \\
\geq & a \nu \int \sigma^{-(\nu-1)}\left[\frac{1}{2}(\phi, \bar{\phi}, \bar{\psi}) M_{4}(\phi, \bar{\phi}, \bar{\psi})^{\mathrm{T}}-\rho_{+} u_{+} \bar{\psi}(\psi-\bar{\psi})-A_{1} \gamma \rho_{+}^{\gamma-1} \phi(\psi-\bar{\psi})\right] d x \\
& +a \nu \int \sigma^{-(\nu-1)}\left[-\left(A_{1} \gamma \widetilde{\rho}^{\gamma-2} \widetilde{u}-A_{1} \gamma \widetilde{\rho}_{+}^{\gamma-2} u_{+}\right) \frac{\phi^{2}}{2}-\left(A_{2} \alpha \widetilde{n}^{\alpha-2} \widetilde{v}-A_{2} \alpha n_{+}^{\alpha-2} u_{+}\right) \frac{\bar{\phi}^{2}}{2}\right. \\
& \left.-\left(A_{1} \gamma \widetilde{\rho}^{\gamma-1}-A_{1} \gamma \rho_{+}^{\gamma-1}\right) \phi \psi-\left(A_{2} \alpha \widetilde{n}^{\alpha-1}-A_{2} \alpha n_{+}^{\alpha-1}\right) \bar{\phi} \bar{\psi}\right] d x-C \frac{\varepsilon}{\delta^{\nu}}\left(\phi^{2}(t, 0)+\bar{\phi}^{2}(t, 0)\right) \\
& -C \varepsilon\left(\left\|\sigma^{-\left(\frac{\nu-2}{2}\right)}(\phi, \psi, \bar{\phi}, \bar{\psi})\right\|^{2}+\left\|\sigma^{-\frac{\nu}{2}}\left(\phi_{x}, \psi_{x}, \bar{\phi}_{x}, \bar{\psi}_{x}\right)\right\|^{2}\right),
\end{aligned}
$$

where the symmetric matrix $\boldsymbol{M}_{\mathbf{4}}$ is defined as

$$
\boldsymbol{M}_{\mathbf{4}}=\left(\begin{array}{ccc}
-A_{2} \gamma \rho_{+}^{\gamma-2} u_{+} & 0 & -A_{1} \gamma \rho_{+}^{\gamma-1} \\
0 & -A_{2} \alpha n_{+}^{\alpha-2} u_{+} & -A_{2} \alpha n_{+}^{\alpha-1} \\
-A_{1} \gamma \rho_{+}^{\gamma-1} & -A_{2} \alpha n_{+}^{\alpha-1} & -\left(\rho_{+}+n_{+}\right) u_{+}
\end{array}\right) .
$$

Under the condition $M_{+}=1$, it is easy to check that three eigenvalues of the matrix $\boldsymbol{M}_{\mathbf{4}}$ satisfy: $\hat{\lambda}_{1}>0$, $\hat{\lambda}_{2}>0, \hat{\lambda}_{3}=0$. Take the coordinate transformation

$$
\left(\begin{array}{l}
\phi \\
\bar{\phi} \\
\bar{\psi}
\end{array}\right)=\boldsymbol{P}\left(\begin{array}{l}
\hat{\rho} \\
\hat{n} \\
\hat{v}
\end{array}\right)
$$

where the matrix $\boldsymbol{P}$ is denoted by

$$
\boldsymbol{P}=\left(\begin{array}{ccc}
r_{11} & r_{21} & -\frac{\rho_{+}}{u_{+}} \\
r_{12} & r_{22} & -\frac{n_{+}}{u_{+}} \\
r_{13} & r_{23} & 1
\end{array}\right) \text { with constants } r_{i j} \text { for } 1 \leq i \leq 2,1 \leq j \leq 3,
$$

such that

$$
(\phi, \bar{\phi}, \bar{\psi}) \boldsymbol{M}_{\mathbf{4}}(\phi, \bar{\phi}, \bar{\psi})^{\mathrm{T}}=(\hat{\rho}, \hat{n}, \hat{v})\left(\begin{array}{ccc}
\hat{\lambda}_{1} & 0 & 0 \\
0 & \hat{\lambda}_{2} & 0 \\
0 & 0 & 0
\end{array}\right)(\hat{\rho}, \hat{n}, \hat{v})^{\mathrm{T}}=\hat{\lambda}_{1} \hat{\rho}^{2}+\hat{\lambda}_{2} \hat{n}^{2}
$$

By (1.13), (4.20), (4.26), (4.28) and (4.29), the third term is estimated as

$$
\begin{aligned}
& -a \nu \int \sigma^{-(\nu-1)} G_{1} d x \\
\geq & a \nu \int \sigma^{-(\nu-1)}\left(\frac{\hat{\lambda}_{1}}{2} \hat{\rho}^{2}+\frac{\hat{\lambda}_{2}}{2} \hat{n}^{2}\right) d x+a \nu \int \sigma^{-(\nu-2)} \frac{A_{1} \gamma(\gamma+1) \rho_{+}^{\gamma}+A_{2} \alpha(\alpha+1) n_{+}^{\alpha}}{2\left|u_{+}\right|^{2}} \hat{v}^{2} d x \\
& +a \nu \int \sigma^{-(\nu-1)} \frac{\rho_{+}\left(u_{+}^{2}-A_{1} \gamma \rho_{+}^{\gamma-1}\right)}{\left|u_{+}\right|} \hat{v}(\psi-\bar{\psi}) d x-C \delta^{\frac{1}{2}}\left\|\sigma^{-\frac{\nu-1}{2}}(\hat{\rho}, \hat{n})\right\|^{2}-C \delta^{\frac{1}{2}}\left\|\sigma^{-\frac{\nu-2}{2}} \hat{v}\right\|^{2} \\
& -C \delta^{\frac{1}{2}}\left\|\sigma^{-\frac{\nu}{2}}(\psi-\bar{\psi})\right\|^{2}-C(\varepsilon+\delta)\left\|\sigma^{-\frac{\nu-2}{2}}(\phi, \psi, \bar{\phi}, \bar{\psi})\right\|^{2}-C \varepsilon\left\|\sigma^{-\frac{\nu}{2}}\left(\phi_{x}, \psi_{x}, \bar{\phi}_{x}, \bar{\psi}_{x}\right)\right\|^{2} \\
& -C \varepsilon \frac{1}{\delta^{\nu}}\left(\phi^{2}(t, 0)+\bar{\phi}^{2}(t, 0)\right),
\end{aligned}
$$


where we have used the following facts:

$$
\begin{aligned}
& -\left(A_{1} \gamma \widetilde{\rho}^{\gamma-1}-A_{1} \gamma \rho_{+}^{\gamma-1}\right) \geq \frac{A_{1} \gamma(\gamma-1) \rho_{+}^{\gamma-1}}{\left|u_{+}\right|} \sigma-C \sigma^{2}, \\
& -\left(A_{2} \alpha \widetilde{n}^{\alpha-1}-A_{2} \alpha n_{+}^{\alpha-1}\right) \geq \frac{A_{2} \alpha(\alpha-1) n_{+}^{\alpha-1}}{\left|u_{+}\right|} \sigma-C \sigma^{2}, \\
& -\left(A_{1} \gamma \widetilde{\rho}^{\gamma-2} \widetilde{u}-A_{1} \gamma \rho_{+}^{\gamma-2} u_{+}\right) \geq A_{1} \gamma(3-\gamma) \rho_{+}^{\gamma-2} \sigma-C \sigma^{2}, \\
& -\left(A_{2} \alpha \widetilde{n}^{\alpha-2} \widetilde{v}-A_{2} \alpha n_{+}^{\alpha-2} u_{+}\right) \geq A_{2} \alpha(3-\alpha) n_{+}^{\alpha-2}-C \sigma^{2} .
\end{aligned}
$$

With the help of (1.21), (3.7), (4.20), (4.21), (4.28), (4.29) and $\psi=\bar{\psi}+(\psi-\bar{\psi})$, we get estimates of the forth, the fifth and seventh terms on the left hand side as

$$
\begin{aligned}
& -a \nu \int \sigma^{-(\nu-1)} G_{2}(t, x) d x \\
\geq & -a^{2} \nu(\nu-1)\left(\frac{\mu}{2}\left\|\sigma^{-\frac{\nu-2}{2}} \psi\right\|^{2}+\frac{n_{+}}{2}\left\|\sigma^{-\frac{\nu-2}{2}} \bar{\psi}\right\|^{2}\right)-C(\delta+\varepsilon)\left\|\sigma^{-\frac{\nu-2}{2}} \bar{\psi}\right\|^{2}-C \varepsilon\left\|\sigma^{-\frac{\nu}{2}} \bar{\psi}_{x}\right\|^{2} \\
\geq & -a^{2} \frac{\mu+n_{+}}{2} \nu(\nu-1)\left\|\sigma^{-\frac{\nu-2}{2}} \bar{\psi}\right\|^{2}-C(\delta+\varepsilon)\left\|\sigma^{-\frac{\nu-2}{2}} \bar{\psi}\right\|^{2}-C \varepsilon\left\|\sigma^{-\frac{\nu}{2}} \bar{\psi}_{x}\right\|^{2}-C \delta\left\|\sigma^{-\frac{\nu}{2}}(\bar{\psi}-\psi)\right\|^{2} \\
\geq & -a^{2} \frac{\mu+n_{+}}{2} \nu(\nu-1)\left\|\sigma^{-\frac{\nu-2}{2}} \hat{v}\right\|^{2}-C \delta^{\frac{1}{2}}\left(\left\|\sigma^{-\frac{\nu-1}{2}}(\hat{\rho}, \hat{n})\right\|^{2}+\left\|\sigma^{-\frac{\nu-2}{2}} \hat{v}\right\|^{2}\right)-C(\delta+\varepsilon)\left\|\sigma^{-\frac{\nu-2}{2}} \bar{\psi}\right\|^{2} \\
& -C \varepsilon\left\|\sigma^{-\frac{\nu}{2}} \bar{\psi}_{x}\right\|^{2}-C \delta\left\|\sigma^{-\frac{\nu}{2}}(\bar{\psi}-\psi)\right\|^{2}, \\
\int & \sigma^{-\nu} n(\bar{\psi}-\psi)^{2} d x \geq\left[n_{+}-C(\delta+\varepsilon)\right]\left\|\sigma^{-\frac{\nu}{2}}(\bar{\psi}-\psi)\right\|^{2}, \\
& \int \sigma^{-\nu} R_{1} d x \\
\geq & a \int \sigma^{-(\nu-2)}\left[\frac{A_{1} \gamma(\gamma-1) \rho_{+}^{\gamma-2}}{2} \phi^{2}+\rho_{+} \psi^{2}+\frac{A_{2} \alpha(\alpha-1) n_{+}^{\alpha-2}}{2} \bar{\phi}^{2}+n_{+} \bar{\psi}^{2}\right] d x \\
& \quad-C(\delta+\varepsilon)\left\|\sigma^{-\frac{\nu-2}{2}}(\phi, \psi, \bar{\phi}, \bar{\psi})\right\|^{2}-C \varepsilon\left\|\sigma^{-\frac{\nu}{2}}\left(\phi_{x}, \psi_{x}, \bar{\phi}_{x}, \bar{\psi}_{x}\right)\right\|^{2}-C \frac{\varepsilon}{\delta^{\nu}}\left(\phi^{2}(t, 0)+\bar{\phi}^{2}(t, 0)\right) \\
\geq & a \frac{A_{1} \gamma(\gamma+1) \rho_{+}^{\gamma}+A_{2} \alpha(\alpha+1) n_{+}^{\alpha}}{2\left|u_{+}\right|^{2}}\left\|\sigma^{-\frac{\nu-2}{2}} \hat{v}\right\|^{2}-C \delta^{\frac{1}{2}}\left\|\sigma^{-\frac{\nu-1}{2}}(\hat{\rho}, \hat{n})\right\|^{2}-C \delta^{\frac{1}{2}}\left\|\sigma^{-\frac{\nu-2}{2}} \hat{v}\right\|^{2} \\
& -C(\delta+\varepsilon)\left\|\sigma^{-\frac{\nu-2}{2}}(\phi, \psi, \bar{\phi}, \bar{\psi})\right\|^{2}-C \varepsilon\left\|\sigma^{-\frac{\nu}{2}}\left(\phi_{x}, \psi_{x}, \bar{\phi}_{x}, \bar{\psi}_{x}\right)\right\|^{2}-C \frac{\varepsilon}{\delta^{\nu}}\left(\phi^{2}(t, 0)+\bar{\phi}^{2}(t, 0)\right) .
\end{aligned}
$$

For $\nu \in(0,3]$, adding (4.33) and (4.35) to the third term in the left side of (4.24) leads to

$$
\begin{aligned}
& -a \nu \int \sigma^{-(\nu-1)} G_{1} d x-a \nu \int \sigma^{-(\nu-1)} G_{2} d x+\int \sigma^{-\nu}(\bar{\psi}-\psi)^{2} d x+\int \sigma^{-\nu} R_{1} d x \\
\geq & c\left\|\sigma^{-\frac{\nu-1}{2}}(\hat{\rho}, \hat{n})\right\|^{2}+\frac{1}{4}\left\{a \frac{A_{1} \gamma(\gamma+1) \rho_{+}^{\gamma}+A_{2} \alpha(\alpha+1) n_{+}^{\alpha}}{2\left|u_{+}\right|^{2}}\left[1+\nu-\frac{\nu(\nu-1)}{2\left(1+b^{2}\right)}\right]\left\|\sigma^{-\frac{\nu-2}{2}} \hat{v}\right\|^{2}\right. \\
& \left.+n_{+}\left\|\sigma^{-\frac{\nu}{2}}(\psi-\bar{\psi})\right\|^{2}\right\}+\int \sigma^{-\nu}(\psi-\bar{\psi}, \hat{v}) M_{5}(\psi-\bar{\psi}, \hat{v})^{\mathrm{T}} d x-C \delta^{\frac{1}{2}}\left\|\sigma^{-\frac{\nu-1}{2}}(\hat{\rho}, \hat{n})\right\|^{2} \\
& -C \delta^{\frac{1}{2}}\left\|\sigma^{-\frac{\nu-2}{2}} \hat{v}\right\|^{2}-C\left(\varepsilon+\delta^{\frac{1}{2}}\right)\left\|\sigma^{-\frac{\nu}{2}}(\psi-\bar{\psi})\right\|^{2}-C(\varepsilon+\delta)\left\|\sigma^{-\frac{\nu}{2}}\left(\phi_{x}, \psi_{x}, \bar{\phi}_{x}, \bar{\psi}_{x}\right)\right\|^{2} \\
& -C \frac{\varepsilon}{\delta^{\nu}}\left(\phi^{2}(t, 0)+\bar{\phi}^{2}(t, 0)\right)-C(\varepsilon+\delta)\left\|\sigma^{-\frac{\nu-2}{2}}(\phi, \psi, \bar{\phi}, \bar{\psi})\right\|^{2} \\
\geq & c\left\|\sigma^{-\frac{\nu-2}{2}}(\phi, \psi, \bar{\phi}, \bar{\psi})\right\|^{2}+c\left\|\sigma^{-\frac{\nu-1}{2}}(\hat{\rho}, \hat{n})\right\|^{2}+c\left\|\sigma^{-\frac{\nu}{2}}(\bar{\psi}-\psi)\right\|^{2} \\
& -C(\delta+\varepsilon)\left\|\sigma^{-\frac{\nu}{2}}\left(\phi_{x}, \psi_{x}, \bar{\phi}_{x}, \bar{\psi}_{x}\right)\right\|^{2}-C \varepsilon \frac{\phi^{2}(t, 0)+\bar{\phi}^{2}(t, 0)}{\delta^{\nu}},
\end{aligned}
$$


where the positive definite matrix $M_{\mathbf{5}}$ is defined by

$$
M_{5}=\left(\begin{array}{cc}
\frac{3}{4} n_{+} & \frac{a \sqrt{\left(\mu+n_{+}\right) n_{+}} b \nu}{2} \sigma \\
\frac{a \sqrt{\left(\mu+n_{+}\right) n_{+}} b \nu}{2} \sigma & \frac{3}{4} a \frac{A_{1} \gamma(\gamma+1) \rho_{+}^{\gamma}+A_{2} \alpha(\alpha+1) n_{+}^{\alpha}}{2\left|u_{+}\right|^{2}}\left[(1+\nu)-\frac{\nu(\nu-1)}{2\left(1+b^{2}\right)}\right] \sigma^{2}
\end{array}\right) .
$$

We will consider the case $\nu \in\left[3,2+\sqrt{8+\frac{1}{1+b^{2}}}\right)$ using the Lemma 4.7 with $\zeta=\sigma^{-(\nu-1)}$. Therefore, with the aid of (2.22), the sixth term is estimated as below:

$$
\begin{aligned}
& \int \sigma^{-\nu}\left(\mu \psi_{x}^{2}+n \bar{\psi}_{x}^{2}\right) d x \\
\geq & a^{2}\left(\mu+n_{+}\right) \frac{(\nu-1)^{2}}{4}\left\|\sigma^{-\frac{\nu-2}{2}} \hat{v}\right\|^{2}-C \delta^{\frac{1}{2}}\left(\left\|\sigma^{-\frac{\nu-2}{2}} \hat{v}\right\|^{2}+\left\|\sigma^{-\frac{\nu-1}{2}}(\hat{\rho}, \hat{n})\right\|^{2}\right) \\
& -C(\delta+\varepsilon)\left\|\sigma^{-\frac{\nu}{2}}\left(\bar{\psi}-\psi, \bar{\psi}_{x}\right)\right\|^{2} .
\end{aligned}
$$

Under the condition $\nu \in(3, \lambda]$, adding (4.36) to (4.38) and using $c|(\phi, \bar{\phi}, \bar{\psi})| \leq|(\hat{\rho}, \hat{n}, \hat{v})| \leq C|(\phi, \bar{\phi}, \bar{\psi})|$, we have for $k=\nu B\left[4\left(1+b^{2}\right) \nu+4 b^{2}+5-\nu^{2}\right]^{-\frac{1}{2}}<1$ that

$$
\begin{aligned}
& -a \nu \int \sigma^{-(\nu-1)} G_{1} d x-a \nu \int \sigma^{-(\nu-1)} G_{2} d x+\int \sigma^{-\nu} n(\bar{\psi}-\psi)^{2} d x+\int \sigma^{-\nu} R_{1} d x \\
& +\int \sigma^{-\nu}\left(\mu \psi_{x}^{2}+n \bar{\psi}_{x}^{2}\right) d x \\
\geq & c \sigma^{-\frac{\nu-1}{2}}(\hat{\rho}, \hat{n}) \|^{2}+\int \sigma^{-\nu}(\psi-\bar{\psi}, \hat{v}) M_{6}(\psi-\bar{\psi}, \hat{v})^{\mathrm{T}} d x+(1-k)\left\{n_{+}\left\|\sigma^{-\frac{\nu}{2}}(\psi-\bar{\psi})\right\|^{2}\right. \\
& \left.+a \frac{A_{1} \gamma(\gamma+1) \rho_{+}^{\gamma}+A_{2} \alpha(\alpha+1) n_{+}^{\alpha}}{2\left|u_{+}\right|^{2}}\left[1+\nu-\frac{\nu(\nu-1)}{2\left(1+b^{2}\right)}+\frac{(\nu-1)^{2}}{4\left(1+b^{2}\right)}\right]\left\|\sigma^{-\frac{\nu-2}{2}} \hat{v}\right\|^{2}\right\} \\
& -C \delta^{\frac{1}{2}}\left(\left\|\sigma^{-\frac{\nu-1}{2}}(\hat{\rho}, \hat{n})\right\|^{2}+\left\|\sigma^{-\frac{\nu-2}{2}} \hat{v}\right\|^{2}\right)-C(\varepsilon+\delta)\left\|\sigma^{-\frac{\nu}{2}}\left(\phi_{x}, \psi_{x}, \bar{\phi}_{x}, \bar{\psi}_{x}\right)\right\|^{2} \\
& -C\left(\varepsilon+\delta^{\frac{1}{2}}\right)\left\|\sigma^{-\frac{\nu}{2}}(\psi-\bar{\psi})\right\|^{2}-C \frac{\varepsilon}{\delta^{\nu}}\left(\phi^{2}(t, 0)+\bar{\phi}^{2}(t, 0)\right)-C(\varepsilon+\delta)\left\|\sigma^{-\frac{\nu-2}{2}}(\phi, \psi, \bar{\phi}, \bar{\psi})\right\|^{2} \\
\geq & c\left\|\sigma^{-\frac{\nu-2}{2}}(\phi, \psi, \bar{\phi}, \bar{\psi})\right\|^{2}+c\left\|\sigma^{-\frac{\nu-1}{2}}(\hat{\rho}, \hat{n})\right\|^{2}+c\left\|\sigma^{-\frac{\nu}{2}}\left(\bar{\psi}-\psi, \psi_{x}, \bar{\psi}_{x}\right)\right\|^{2} \\
& -C(\delta+\varepsilon)\left\|\sigma^{-\frac{\nu}{2}}\left(\phi_{x}, \bar{\phi}_{x}\right)\right\|^{2}-C \frac{\varepsilon}{\delta^{\nu}}\left(\phi^{2}(t, 0)+\bar{\phi}^{2}(t, 0)\right),
\end{aligned}
$$

where $\delta^{\frac{1}{2}}$ and $\varepsilon$ are small enough, and the positive definite matrix $\boldsymbol{M}_{\mathbf{6}}$ is defined as

$$
\boldsymbol{M}_{\mathbf{6}}=\left(\begin{array}{cc}
\frac{k n_{+}}{\frac{a \nu \sqrt{\left(\mu+n_{+}\right) n_{+}} b}{2}} \sigma \\
\frac{a \nu \sqrt{\left(\mu+n_{+}\right) n_{+} b}}{2} \sigma & k a \frac{A_{1} \gamma(\gamma+1) \rho_{+}^{\gamma}+A_{2} \alpha(\alpha+1) n_{+}^{\alpha}}{2\left|u_{+}\right|^{2}}\left[1+\nu-\frac{\nu(\nu-1)}{2\left(1+b^{2}\right)}+\frac{(\nu-1)^{2}}{4\left(1+b^{2}\right)}\right] \sigma^{2}
\end{array}\right) .
$$

By (1.12), (1.13), (4.29), Cauchy-Schwarz inequality and $u_{+}^{2}=\frac{A_{1} \gamma \rho_{+}^{\gamma}+A_{2} \alpha n_{+}^{\alpha}}{\rho_{+}+n_{+}}$, we estimate terms on the right hand side as

$$
\begin{aligned}
& \left|\int \sigma^{-\nu} R_{2} d x+\int \sigma^{-\nu} R_{3} d x\right| \\
\leq & \left|a \int \sigma^{-(\nu-2)} \frac{\left(\rho_{+}+n_{+}\right) u_{+}^{2}-\left(A_{1} \gamma \rho_{+}^{\gamma}+A_{2} \alpha n_{+}^{\alpha}\right)}{\left|u_{+}\right|^{2}} \hat{v}^{2} d x\right|+C \delta^{\frac{1}{2}}\left\|\sigma^{-\frac{\nu-1}{2}}(\hat{\rho}, \hat{n})\right\|^{2} \\
& +C \delta^{\frac{1}{2}}\left\|\sigma^{-\frac{\nu-2}{2}}(\phi, \psi, \bar{\phi}, \bar{\psi})\right\|^{2}+C \delta\left\|\sigma^{-\frac{\nu}{2}}\left(\psi-\bar{\psi}, \bar{\psi}_{x}\right)\right\|^{2} \\
\leq & C \delta^{\frac{1}{2}}\left\|\sigma^{-\frac{\nu-1}{2}}(\hat{\rho}, \hat{n})\right\|^{2}+C \delta^{\frac{1}{2}}\left\|\sigma^{-\frac{\nu-2}{2}}(\phi, \psi, \bar{\phi}, \bar{\psi})\right\|^{2}+C \delta\left\|\sigma^{-\frac{\nu}{2}}\left(\bar{\psi}_{x}, \bar{\psi}-\psi\right)\right\|^{2} .
\end{aligned}
$$


Finally, with $\delta^{\frac{1}{2}}$ and $\varepsilon$ small enough, and combining (4.25)-(4.41), we obtain

$$
\begin{aligned}
& \frac{d}{d t} \int \sigma^{-\nu}\left(\mathcal{E}_{1}+\mathcal{E}_{2}\right) d x+c\left\|\sigma^{-\frac{\nu-2}{2}}(\phi, \psi, \bar{\phi}, \bar{\psi})\right\|^{2}+c\left\|\sigma^{-\frac{\nu}{2}}\left(\psi_{x}, \bar{\psi}_{x}, \bar{\psi}-\psi\right)\right\|^{2} \\
& +\frac{c}{\delta^{\nu}}\left[\phi^{2}(0, t)+\bar{\phi}^{2}(t, 0)\right] \\
\leq & C(\delta+\varepsilon)\left\|\sigma^{-\frac{\nu}{2}}\left(\phi_{x}, \bar{\phi}_{x}\right)\right\|^{2} .
\end{aligned}
$$

Multiplying (4.42) by $(1+\delta \tau)^{\xi}$ and integrating the resulted equation in $\tau$ over $[0, t]$, we obtain

$$
\begin{aligned}
& (1+\delta \tau)^{\xi}\left\|\sigma^{-\frac{\nu}{2}}(\phi, \psi, \bar{\phi}, \bar{\psi})\right\|^{2}+\int_{0}^{t}(1+\delta \tau)^{\xi}\left\|\sigma^{-\frac{\nu-2}{2}}(\phi, \psi, \bar{\phi}, \bar{\psi})\right\|^{2} d \tau \\
& +\int_{0}^{t}(1+\delta \tau)^{\xi}\left\|\sigma^{-\frac{\nu}{2}}\left(\psi_{x}, \bar{\psi}_{x}, \bar{\psi}-\psi\right)\right\|^{2} d \tau+\frac{1}{\delta^{\nu}} \int_{0}^{t}(1+\delta \tau)^{\xi}\left(\phi^{2}(t, 0)+\bar{\phi}^{2}(t, 0)\right) d \tau \\
\leq & C\left\|\sigma^{-\frac{\lambda}{2}}\left(\phi_{0}, \psi_{0}, \bar{\phi}_{0}, \bar{\psi}_{0}\right)\right\|^{2}+C(\delta+\varepsilon) \int_{0}^{t}(1+\delta \tau)^{\xi}\left\|\sigma^{-\frac{\nu}{2}}\left(\phi_{x}, \bar{\phi}_{x}\right)\right\|^{2} d \tau \\
& +C \delta \xi \int_{0}^{t}(1+\delta \tau)^{\xi-1}\left\|\sigma^{-\frac{\nu}{2}}(\phi, \psi, \bar{\phi}, \bar{\psi})\right\|^{2} d \tau,
\end{aligned}
$$

which leads to the completeness for the proof of Lemma 4.8.

In order to show Proposition 4.5, we need to obtain the weighted energy estimates of $\left(\phi_{x}, \psi_{x}, \bar{\phi}_{x}, \bar{\psi}_{x}\right)$.

Lemma 4.9. Under the same conditions in Proposition 4.5, then the solution $(\phi, \psi, \bar{\phi}, \bar{\psi})$ to the IBVP (3.3)-(3.7) satisfies for $t \in[0, T]$ that

$$
\begin{aligned}
& (1+\delta t)^{\xi}\left\|\sigma^{-\frac{\nu}{2}}\left(\phi_{x}, \bar{\phi}_{x}\right)\right\|^{2}+\int_{0}^{t}(1+\delta \tau)^{\xi}\left\|\sigma^{-\frac{\nu}{2}}\left(\phi_{x}, \bar{\phi}_{x}\right)\right\|^{2} d \tau \\
\leq & C\left(\left\|\sigma^{-\frac{\lambda}{2}}\left(\phi_{0}, \bar{\phi}_{0}, \psi_{0}, \bar{\psi}_{0}\right)\right\|^{2}+\left\|\sigma^{-\frac{\lambda}{2}}\left(\phi_{0 x}, \bar{\phi}_{0 x}\right)\right\|^{2}\right)+C(\varepsilon+\delta) \int(1+\delta \tau)^{\xi}\left\|\sigma^{-\frac{\nu}{2}}\left(\psi_{x x}, \bar{\psi}_{x x}\right)\right\|^{2} d \tau \\
& +\delta \xi \int_{0}^{t}(1+\delta \tau)^{\xi-1}\left\|\sigma^{-\frac{\nu}{2}}\left(\phi, \psi, \phi_{x}, \bar{\phi}, \bar{\psi}, \bar{\phi}_{x}\right)\right\|^{2} d \tau,
\end{aligned}
$$

with $\xi \geq 0$.

Proof. Adding (3.25)-(3.28) together, then multiplying the resulted equation by $\sigma^{-\nu}$ and integrating over $\mathbb{R}_{+}$with the space weight function $\sigma \geq 0$ satisfying (1.14) and (2.28), we obtain

$$
\begin{aligned}
& \frac{d}{d t} \int \sigma^{-\nu}\left(\mu \frac{\phi_{x}^{2}}{2}+\frac{\bar{\phi}_{x}^{2}}{2}+\widetilde{\rho}^{2} \phi_{x} \psi+\widetilde{n} \bar{\phi}_{x} \bar{\psi}\right) d x-\left[\sigma^{-\nu}\left(\mu u \frac{\phi_{x}^{2}}{2}+v \frac{\bar{\phi}_{x}^{2}}{2}-\widetilde{\rho}^{2} \phi_{t} \psi-\widetilde{n} \bar{\phi}_{t} \bar{\psi}\right)\right](t, 0) \\
& -a \nu \int \sigma^{-(\nu-1)}\left[\mu u \frac{\phi_{x}^{2}}{2}+v \frac{\bar{\phi}_{x}^{2}}{2}-\widetilde{\rho}^{2} \phi_{t} \psi-\widetilde{n} \bar{\phi}_{t} \bar{\psi}\right] d x+\int \sigma^{-\nu}\left(\widetilde{\rho}^{2} \frac{p_{1}^{\prime}(\rho)}{\rho} \phi_{x}^{2}+\widetilde{n} \frac{p_{2}^{\prime}(n)}{n} \bar{\phi}_{x}^{2}\right) d x \\
= & \sum_{i=1}^{6} \mathcal{J}_{i},
\end{aligned}
$$

where

$$
\begin{aligned}
& \mathcal{J}_{1}=-\int \sigma^{-\nu}\left[\widetilde{\rho}^{2}\left(\phi_{t}+u \phi_{x}\right) \psi_{x}+\widetilde{n}\left(\bar{\phi}_{t}+v \bar{\phi}_{x}\right) \bar{\psi}_{x}+2 \widetilde{\rho} \widetilde{\rho}_{x} \phi_{t} \psi+\widetilde{n}_{x} \bar{\phi}_{t} \bar{\psi}\right] d x \\
& \mathcal{J}_{2}=\int \sigma^{-\nu}\left[-\mu \phi \phi_{x} \psi_{x x}-\bar{\phi} \bar{\phi}_{x} \bar{\psi}_{x x}+\mu \widetilde{\rho}^{2}\left(\frac{1}{\rho}-\frac{1}{\widetilde{\rho}}\right) \phi_{x} \psi_{x x}+\widetilde{n}\left(\frac{1}{n}-\frac{1}{\widetilde{n}}\right)\left(\widetilde{n} \bar{\psi}_{x}\right)_{x} \bar{\phi}_{x}\right] d x, \\
& \mathcal{J}_{3}=\int \sigma^{-\nu}\left[\widetilde{\rho}^{2} \frac{n}{\rho}(\bar{\psi}-\psi) \phi_{x}-\widetilde{n}(\bar{\psi}-\psi) \bar{\phi}_{x}\right] d x
\end{aligned}
$$




$$
\begin{aligned}
\mathcal{J}_{4}= & -\int \sigma^{-\nu}\left(\frac{3}{2} \mu \psi_{x} \frac{\phi_{x}^{2}}{2}+\frac{3}{2} \bar{\psi}_{x} \bar{\phi}_{x}^{2}\right) d x+\int \sigma^{-\nu} \widetilde{n} \frac{\left(\bar{\phi} \bar{\psi}_{x}\right)_{x}}{n} \bar{\phi}_{x} d x, \quad \mathcal{J}_{5}=\int \sigma^{-\nu}\left(F_{1} \widetilde{\rho}^{2} \phi_{x}+F_{2} \widetilde{n} \bar{\phi}_{x}\right) d x, \\
\mathcal{J}_{6}= & -\int \sigma^{-\nu}\left[\mu\left(\frac{1}{2} \phi_{x} \widetilde{u}_{x}+\psi_{x} \widetilde{\rho}_{x}\right) \phi_{x}+\mu\left(\phi \widetilde{u}_{x}+\psi \widetilde{\rho}_{x}\right)_{x} \phi_{x}\right. \\
& \left.-\widetilde{n}_{x} \bar{\phi}_{x} \bar{\psi}_{x x}+\left(\frac{1}{2} \bar{\phi}_{x} \widetilde{v}_{x}+\bar{\psi}_{x} \widetilde{n}_{x}\right) \bar{\phi}_{x}-\left(\bar{\phi} \widetilde{v}_{x}+\bar{\psi} \widetilde{n}_{x}\right)_{x} \bar{\phi}_{x}\right] d x .
\end{aligned}
$$

Using (1.12), (3.7), and (4.20), we obtain the estimates for terms on the left hand side as below

$$
\begin{aligned}
- & {\left[\sigma^{-\nu}\left(\mu u \frac{\phi_{x}^{2}}{2}+v \frac{\bar{\phi}_{x}^{2}}{2}-\widetilde{\rho} \phi_{x} \psi-\widetilde{n} \bar{\phi}_{x} \bar{\psi}\right)\right](t, 0) \geq \frac{c}{\delta^{\nu}}\left(\phi_{x}^{2}(t, 0)+\bar{\phi}_{x}^{2}(t, 0)\right) \geq 0, } \\
- & a \nu \int \sigma^{-(\nu-1)}\left(\mu u \frac{\phi_{x}^{2}}{2}+v \frac{\bar{\phi}_{x}^{2}}{2}\right) d x \\
\geq & \frac{a \nu\left|u_{+}\right|}{2} \int \sigma^{-(\nu-1)}\left(\mu \phi_{x}^{2}+\bar{\phi}_{x}^{2}\right) d x-C(\varepsilon+\delta)\left\|\sigma^{-\frac{\nu-1}{2}}\left(\phi_{x}, \bar{\phi}_{x}\right)\right\|^{2}, \\
& -a \nu \int \sigma^{-(\nu-1)}\left(-\widetilde{\rho}^{2} \phi_{t} \psi-\widetilde{n} \bar{\phi}_{t} \bar{\psi}\right) d x \\
\geq & -C \varepsilon\left\|\sigma^{-\frac{\nu}{2}}\left(\phi_{x}, \bar{\phi}_{x}\right)\right\|^{2}-C\left\|\sigma^{-\frac{\nu}{2}}(\phi, \psi, \bar{\phi}, \bar{\psi})\right\|^{2}-C\left\|\sigma^{-\frac{\nu}{2}}\left(\psi_{x}, \bar{\psi}_{x}\right)\right\|^{2}, \\
& \int \sigma^{-\nu}\left(\widetilde{\rho}^{2} \frac{p_{1}^{\prime}(\rho)}{\rho} \phi_{x}^{2}+\widetilde{n} \frac{p_{2}^{\prime}(n)}{n} \bar{\phi}_{x}^{2}\right) d x \geq \frac{A_{1} \gamma \rho_{+}^{\gamma}}{2}\left\|\sigma^{-\frac{\nu}{2}} \phi_{x}\right\|^{2}+\frac{A_{2} \alpha n_{+}^{\alpha-1}}{2}\left\|\sigma^{-\frac{\nu}{2}} \bar{\phi}_{x}\right\|^{2},
\end{aligned}
$$

under the condition $\delta$ and $\varepsilon$ small enough.

We turn to estimate terms on the right hand side of (4.45). With the help of (1.12), (4.20), Young inequality and Cauchy-Schwarz inequality, we gain

$$
\begin{aligned}
&\left|\mathcal{J}_{1}\right| \leq C \delta\left\|\sigma^{-\frac{\nu}{2}}\left(\phi_{x}, \bar{\phi}_{x}\right)\right\|^{2}+C\left\|\sigma^{-\frac{\nu}{2}}\left(\psi_{x}, \bar{\psi}_{x}\right)\right\|^{2}+C\left\|\sigma^{-\frac{\nu-2}{2}}(\phi, \psi, \bar{\psi}, \bar{\psi})\right\|^{2}, \\
&\left|\mathcal{J}_{2}\right| \leq C\left.C(\phi, \bar{\phi}) \|_{L^{\infty}}+\delta\right)\left\|\sigma^{-\frac{\nu}{2}}\left(\phi_{x}, \bar{\phi}_{x}, \psi_{x x}, \bar{\psi}_{x x}\right)\right\|^{2}+C \delta^{2}\left\|\sigma^{-\frac{\nu}{2}} \bar{\psi}_{x}\right\|^{2} \\
& \leq C(\delta+\varepsilon)\left\|\sigma^{-\frac{\nu}{2}}\left(\phi_{x}, \bar{\phi}_{x}\right)\right\|^{2}+C(\delta+\varepsilon)\left\|\sigma^{-\frac{\nu}{2}}\left(\psi_{x x}, \bar{\psi}_{x x}\right)\right\|^{2}+C \delta\left\|\sigma^{-\frac{\nu}{2}} \bar{\psi}_{x}\right\|^{2}, \\
&\left|\mathcal{J}_{3}\right| \leq \frac{A_{1} \gamma \rho_{+}^{\gamma}}{8}\left\|\sigma^{-\frac{\nu}{2}} \phi_{x}\right\|^{2}+\frac{A_{2} \alpha n_{+}^{\alpha-1}}{8}\left\|\sigma^{-\frac{\nu}{2}} \bar{\phi}_{x}\right\|^{2}+C\left\|\sigma^{-\frac{\nu}{2}}(\bar{\psi}-\psi)\right\|^{2}, \\
&\left|\mathcal{J}_{4}\right| \leq C\left\|\left(\psi_{x}, \bar{\psi}_{x}\right)\right\|_{L^{\infty}}\left\|\sigma^{-\frac{\nu}{2}}\left(\phi_{x}, \bar{\phi}_{x}\right)\right\|^{2}+C\|\phi\|_{L^{\infty}}\left\|\sigma^{-\frac{\nu}{2}}\left(\bar{\phi}_{x}, \bar{\psi}_{x}\right)\right\|^{2} \\
& \leq C \varepsilon\left\|\sigma^{-\frac{\nu}{2}}\left(\phi_{x}, \bar{\phi}_{x}\right)\right\|^{2}+C \varepsilon\left\|\sigma^{-\frac{\nu}{2}}\left(\psi_{x x}, \bar{\psi}_{x x}\right)\right\|^{2}+C \varepsilon\left\|\sigma^{-\frac{\nu}{2}}\left(\psi_{x}, \bar{\psi}_{x}\right)\right\|^{2}, \\
&\left|\mathcal{J}_{5}\right| \leq C \delta\left\|\sigma^{-\frac{\nu}{2}}\left(\phi_{x}, \bar{\phi}_{x}\right)\right\|^{2}+C \delta\left\|\sigma^{-\frac{\nu-2}{2}}(\phi, \psi, \bar{\phi}, \bar{\psi})\right\|^{2}, \\
&\left|\mathcal{J}_{6}\right| \leq C \delta\left\|\sigma^{-\frac{\nu}{2}}\left(\phi_{x}, \bar{\phi}_{x}\right)\right\|^{2}+C \delta^{2}\left\|\sigma^{-\frac{\nu}{2}} \bar{\psi}_{x x}\right\|^{2}+C \delta\left\|\sigma^{-\frac{\nu}{2}}\left(\psi_{x}, \bar{\psi}_{x}\right)\right\|^{2}+C \delta\left\|\sigma^{-\frac{\nu-2}{2}}(\phi, \bar{\phi}, \psi, \bar{\psi})\right\|^{2} .
\end{aligned}
$$

Finally, the substitution of (4.46)-(4.55) into (4.45) for $\delta$ and $\varepsilon$ small enough leads to that

$$
\begin{aligned}
& \frac{d}{d t} \int \sigma^{-\nu}\left[\mu \frac{\phi_{x}^{2}}{2}+\frac{\bar{\phi}_{x}^{2}}{2}+\widetilde{\rho}^{2} \phi_{x} \psi+\widetilde{n} \bar{\phi}_{x} \bar{\psi}\right] d x+c\left\|\sigma^{-\frac{\nu-1}{2}}\left(\phi_{x}, \bar{\phi}_{x}\right)\right\|^{2} \\
& +\frac{A_{1} \gamma \rho_{+}^{\gamma}}{4}\left\|\sigma^{-\frac{\nu}{2}} \phi_{x}\right\|^{2}+\frac{A_{2} \alpha n_{+}^{\alpha-1}}{4}\left\|\sigma^{-\frac{\nu}{2}} \bar{\phi}_{x}\right\|^{2} \\
\leq & C(\varepsilon+\delta)\left\|\sigma^{-\frac{\nu}{2}}\left(\psi_{x x}, \bar{\psi}_{x x}\right)\right\|^{2}+C\left\|\sigma^{-\frac{\nu-2}{2}}(\phi, \psi, \bar{\phi}, \bar{\psi})\right\|^{2}+C\left\|\sigma^{-\frac{\nu}{2}}\left(\psi_{x}, \bar{\psi}_{x}, \bar{\psi}-\psi\right)\right\|^{2} .
\end{aligned}
$$


Multiplying (4.56) by $(1+\delta \tau)^{\xi}$ and integrating the resulted equation in $\tau$ over $[0, t]$ imply

$$
\begin{aligned}
& (1+\delta t)^{\xi}\left\|\sigma^{-\frac{\nu}{2}}\left(\phi_{x}, \bar{\phi}_{x}\right)\right\|^{2}+\int_{0}^{t}(1+\delta \tau)^{\xi}\left\|\sigma^{-\frac{\nu-1}{2}}\left(\phi_{x}, \bar{\phi}_{x}\right)\right\|^{2} d \tau+\int_{0}^{t}(1+\delta \tau)^{\xi}\left\|\sigma^{-\frac{\nu}{2}}\left(\phi_{x}, \bar{\phi}_{x}\right)\right\|^{2} d \tau \\
\leq & C\left\|\sigma^{-\frac{\lambda}{2}}\left(\phi_{0}, \psi_{0}, \phi_{0 x}, \bar{\phi}_{0}, \bar{\psi}_{0}, \bar{\phi}_{0 x}\right)\right\|^{2}+C(\varepsilon+\delta) \int(1+\delta \tau)^{\xi}\left\|\sigma^{-\frac{\nu}{2}}\left(\psi_{x x}, \bar{\psi}_{x x}\right)\right\|^{2} d \tau \\
& +\delta \xi \int_{0}^{t}(1+\delta \tau)^{\xi-1}\left\|\sigma^{-\frac{\nu}{2}}\left(\phi, \psi, \phi_{x}, \bar{\phi}, \bar{\psi}, \bar{\phi}_{x}\right)\right\|^{2} d \tau,
\end{aligned}
$$

with the help of Cauchy-Schwarz inequality and (4.23).

Lemma 4.10. Under the same conditions in Proposition 4.5, then the solution $(\phi, \psi, \bar{\phi}, \bar{\psi})$ to the IBVP (3.3)-(3.7) satisfies for $t \in[0, T]$ that

$$
\begin{aligned}
& (1+\delta t)^{\xi}\left\|\sigma^{-\frac{\nu}{2}}\left(\psi_{x}, \bar{\psi}_{x}\right)\right\|^{2}+\int_{0}^{t}(1+\delta \tau)^{\xi}\left\|\sigma^{-\frac{\nu}{2}}\left(\psi_{x x}, \bar{\psi}_{x x}\right)\right\|^{2} d \tau \\
\leq & C\left\|\sigma^{-\frac{\lambda}{2}}\left(\phi_{0}, \psi_{0}, \phi_{0 x}, \psi_{0 x}, \bar{\phi}_{0}, \bar{\psi}_{0}, \bar{\phi}_{0 x}, \bar{\psi}_{0 x}\right)\right\|^{2} \\
& +C \delta \xi \int_{0}^{t}(1+\delta \tau)^{\xi-1}\left\|\sigma^{-\frac{\nu}{2}}\left(\phi, \psi, \phi_{x}, \psi_{x}, \bar{\phi}, \bar{\psi}, \bar{\phi}_{x}, \bar{\psi}_{x}\right)\right\|^{2} d \tau .
\end{aligned}
$$

with $\xi \geq 0$.

Proof. Multiplying $(3.3)_{2}$ by $-\sigma^{-\nu} \psi_{x x},(3.3)_{4}$ by $-\sigma^{-\nu} \bar{\psi}_{x x}$, respectively for the function $\sigma$ satisfying (1.14) and (2.28), then adding them together and integrating the resulted equation in $x$ over $\mathbb{R}_{+}$lead to

$$
\frac{d}{d t} \int \sigma^{-\nu}\left(\frac{\psi_{x}^{2}}{2}+\frac{\bar{\psi}_{x}^{2}}{2}\right) d x-a \nu \int \sigma^{-(\nu-1)}\left(\psi_{t} \psi_{x}+\bar{\psi}_{t} \bar{\psi}_{x}\right) d x+\int \sigma^{-\nu}\left(\frac{\mu}{\rho} \psi_{x x}^{2}+\bar{\psi}_{x x}^{2}\right) d x=\sum_{i=1}^{3} \mathcal{K}_{i},
$$

where

$$
\begin{aligned}
\mathcal{K}_{1}= & \int \sigma^{-\nu}\left[u \psi_{x} \psi_{x x}-\frac{n}{\rho}(\bar{\psi}-\psi) \psi_{x x}+\frac{p_{1}^{\prime}(\rho)}{\rho} \phi_{x} \psi_{x x}+v \bar{\psi}_{x} \bar{\psi}_{x x}+(\bar{\psi}-\psi) \bar{\psi}_{x x}+\frac{p_{2}^{\prime}(n)}{n} \bar{\phi}_{x} \bar{\psi}_{x x}\right] d x, \\
\mathcal{K}_{2}= & \int \sigma^{-\nu}\left[\widetilde{u}_{x} \psi \psi_{x x}-\mu \widetilde{u}_{x x}\left(\frac{1}{\rho}-\frac{1}{\widetilde{\rho}}\right) \psi_{x x}+\left(\frac{p_{1}^{\prime}(\rho)}{\rho}-\frac{p_{1}^{\prime}(\widetilde{\rho})}{\widetilde{\rho}}\right) \psi_{x x} \widetilde{\rho}_{x}-\left(\frac{n}{\rho}-\frac{\widetilde{n}}{\widetilde{\rho}}\right)(\widetilde{v}-\widetilde{u}) \psi_{x x}+\widetilde{v}_{x} \bar{\psi} \bar{\psi}_{x x}\right. \\
& \left.+\left(\widetilde{n} \widetilde{v}_{x}\right)_{x}\left(\frac{1}{n}-\frac{1}{\widetilde{n}}\right) \bar{\psi}_{x x}+\left(\frac{p_{2}^{\prime}(n)}{n}-\frac{p_{2}^{\prime}(\widetilde{n})}{\widetilde{n}}\right) \bar{\psi}_{x x} \widetilde{n}_{x}-\frac{\widetilde{n}_{x}}{\widetilde{n}} \bar{\psi}_{x} \bar{\psi}_{x x}-\frac{\left(\bar{\phi} \widetilde{v}_{x}\right)_{x}}{n} \bar{\psi}_{x x}\right] d x, \\
\mathcal{K}_{3}= & -\int \sigma^{-\nu}\left[\frac{\left(\bar{\phi} \bar{\psi}_{x}\right)_{x}}{n} \bar{\psi}_{x x}+\left(\frac{1}{n}-\frac{1}{\widetilde{n}}\right)\left(\widetilde{n} \bar{\psi}_{x}\right)_{x} \bar{\psi}_{x x}\right] d x .
\end{aligned}
$$

First, we estimate terms in the left side of (4.59). The second term is estimated as follows:

$$
\begin{aligned}
& a \nu \int \sigma^{-(\nu-1)}\left(\psi_{t} \psi_{x}+\bar{\psi}_{t} \bar{\psi}_{x}\right) d x \\
\leq & C \delta\left\|\sigma^{-\frac{\nu}{2}}\left(\psi_{x x}, \bar{\psi}_{x x}\right)\right\|^{2}+C \delta\left\|\sigma^{-\frac{\nu}{2}}\left(\phi_{x}, \psi_{x}, \bar{\phi}_{x}, \bar{\psi}_{x}, \bar{\psi}-\psi\right)\right\|^{2}+C \delta\left\|\sigma^{-\frac{\nu-2}{2}}(\phi, \psi, \bar{\phi}, \bar{\psi})\right\|^{2},
\end{aligned}
$$

where we have used $(1.12),(3.3)_{2},(3.3)_{4},(4.20)$ and Cauchy-Schwarz inequality. The third term is estimate as follows:

$$
\begin{aligned}
\int \sigma^{-\nu}\left(\frac{\mu}{\rho} \psi_{x x}^{2}+\bar{\psi}_{x x}^{2}\right) d x & \geq\left[\frac{\mu}{\rho_{+}}-C(\varepsilon+\delta)\right]\left\|\sigma^{-\frac{\nu}{2}} \psi_{x x}\right\|^{2}+\left\|\sigma^{-\frac{\nu}{2}} \bar{\psi}_{x x}\right\|^{2} \\
& \geq \frac{\mu}{2 \rho_{+}}\left\|\sigma^{-\frac{\nu}{2}} \psi_{x x}\right\|^{2}+\left\|\sigma^{-\frac{\nu}{2}} \bar{\psi}_{x x}\right\|^{2}
\end{aligned}
$$


if $\delta$ and $\varepsilon$ are small enough.

We turn to estimate terms on the right hand side of (4.59). With the help of (1.12), (4.20) and CauchySchwarz inequality, we obtain

$$
\begin{gathered}
\left|\mathcal{K}_{1}\right| \leq \frac{\mu}{8 \rho_{+}}\left\|\sigma^{-\frac{\nu}{2}} \psi_{x x}\right\|^{2}+\frac{1}{8}\left\|\sigma^{-\frac{\nu}{2}} \bar{\psi}_{x x}\right\|^{2}+C\left\|\sigma^{-\frac{\nu}{2}}\left(\phi_{x}, \psi_{x}, \bar{\phi}_{x}, \bar{\psi}_{x}, \bar{\psi}-\psi\right)\right\|^{2}, \\
\left|\mathcal{K}_{2}\right| \leq C \delta\left\|\sigma^{-\frac{\nu}{2}}\left(\psi_{x x}, \bar{\psi}_{x x}\right)\right\|^{2}+C \delta\left\|\sigma^{-\frac{\nu-2}{2}}(\phi, \psi, \bar{\phi}, \bar{\psi})\right\|^{2}+C \delta\left\|\sigma^{-\frac{\nu}{2}}\left(\bar{\phi}_{x}, \bar{\psi}_{x}\right)\right\|^{2}, \\
\left|\mathcal{K}_{3}\right| \leq C(\varepsilon+\delta)\left\|\sigma^{-\frac{\nu}{2}}\left(\bar{\psi}_{x}, \bar{\psi}_{x x}\right)\right\|^{2} .
\end{gathered}
$$

Finally, we substitute (4.60)-(4.64) into (4.59) to gain under the condition $\delta$ and $\varepsilon$ small enough that

$$
\begin{aligned}
& \frac{d}{d t} \int \sigma^{-\nu}\left(\frac{\psi_{x}^{2}}{2}+\frac{\bar{\psi}_{x}^{2}}{2}\right) d x+\frac{\mu}{4 \rho_{+}}\left\|\sigma^{-\frac{\nu}{2}} \psi_{x x}\right\|^{2}+\frac{1}{4}\left\|\sigma^{-\frac{\nu}{2}} \bar{\psi}_{x x}\right\|^{2} \\
\leq & C\left\|\sigma^{-\frac{\nu}{2}}\left(\phi_{x}, \psi_{x}, \bar{\phi}_{x}, \bar{\psi}_{x}, \bar{\psi}-\psi\right)\right\|^{2}+C \delta\left\|\sigma^{-\frac{\nu-2}{2}}(\phi, \psi, \bar{\phi}, \bar{\psi})\right\|^{2}
\end{aligned}
$$

Multiplying (4.65) by $(1+\delta \tau)^{\xi}$ and integrating the resulted inequality in $\tau$ over $\tau \in[0, t]$, we can obtain

$$
\begin{aligned}
& (1+\delta t)^{\xi}\left\|\sigma^{-\frac{\nu}{2}}\left(\psi_{x}, \bar{\psi}_{x}\right)\right\|^{2}+\int_{0}^{t}(1+\delta \tau)^{\xi}\left\|\sigma^{-\frac{\nu}{2}}\left(\psi_{x x}, \bar{\psi}_{x x}\right)\right\|^{2} d \tau \\
\leq & C\left\|\sigma^{-\frac{\lambda}{2}}\left(\phi_{0}, \psi_{0}, \bar{\phi}_{0}, \bar{\psi}_{0}\right)\right\|_{1}^{2}+C \delta \xi \int_{0}^{t}(1+\delta \tau)^{\xi-1}\left\|\sigma^{-\frac{\nu}{2}}(\phi, \psi, \bar{\phi}, \bar{\psi})\right\|_{1}^{2} d \tau,
\end{aligned}
$$

where we have used (4.23), (4.44) and the smallness of $\delta$ and $\varepsilon$. Hence we finish the proof.

Proof of Proposition 4.5 We sum up the estimates (4.23), (4.44) and (4.58) and take $\delta$ and $\varepsilon$ suitably small to obtain

$$
\begin{aligned}
& (1+\delta t)^{\xi}\left\|\sigma^{-\frac{\nu}{2}}(\phi, \psi, \bar{\phi}, \bar{\psi})\right\|_{1}^{2}+\int_{0}^{t}(1+\delta \tau)^{\xi}\left\|\sigma^{-\frac{\nu-2}{2}}(\phi, \psi, \bar{\phi}, \bar{\psi})\right\|^{2} d \tau \\
& +\int_{0}^{t}(1+\delta \tau)^{\xi}\left\|\sigma^{-\frac{\nu}{2}}\left(\phi_{x}, \psi_{x}, \bar{\phi}_{x}, \bar{\psi}_{x}\right)\right\|^{2}+C \int_{0}^{t}(1+\delta \tau)^{\xi}\left\|\sigma^{-\frac{\nu}{2}}\left(\psi_{x x}, \bar{\psi}_{x x}, \bar{\psi}-\psi\right)\right\|^{2} \\
& +C \frac{1}{\delta^{\nu}} \int_{0}^{t}(1+\delta \tau)^{\xi}\left(\phi^{2}(0, t)+\bar{\phi}^{2}(0, t)\right) d \tau \\
& \leq C\left\|\sigma^{-\frac{\lambda}{2}}\left(\phi_{0}, \psi_{0}, \bar{\phi}_{0}, \bar{\psi}_{0}\right)\right\|_{1}^{2}+C \delta \xi \int_{0}^{t}(1+\delta \tau)^{\xi-1}\left\|\sigma^{-\frac{\nu}{2}}(\phi, \psi, \bar{\phi}, \bar{\psi})\right\|_{1}^{2} d \tau,
\end{aligned}
$$

for a positive constant $C>0$ independent of $T, \nu$ and $\xi$. Applying induction arguments in $[2,17,24]$ to (4.67), we have

$$
\begin{aligned}
& (1+\delta t)^{\frac{\lambda-\nu}{2}+\beta}\left\|\sigma^{-\frac{\nu}{2}}\left(\phi, \psi, \phi_{x}, \psi_{x}, \bar{\phi}, \bar{\psi}, \bar{\phi}_{x}, \bar{\psi}_{x}\right)\right\|^{2}+\int_{0}^{t}(1+\delta \tau)^{\frac{\lambda-\nu}{2}+\beta}\left\|\sigma^{-\frac{\nu-2}{2}}(\phi, \bar{\phi}, \psi, \bar{\psi})\right\|^{2} \\
& +\int_{0}^{t}(1+\delta \tau)^{\frac{\lambda-\nu}{2}+\beta}\left\|\sigma^{-\frac{\nu}{2}}\left(\phi_{x}, \psi_{x}, \psi_{x x}, \bar{\phi}_{x}, \bar{\psi}_{x}, \bar{\psi}_{x x}, \bar{\psi}-\psi\right)\right\|^{2} \\
\leq & C(1+\delta t)^{\beta}\left\|\sigma^{-\frac{\lambda}{2}}\left(\phi_{0}, \psi_{0}, \phi_{0 x}, \psi_{0 x}, \bar{\phi}_{0}, \bar{\psi}_{0}, \bar{\phi}_{0 x}, \bar{\psi}_{0 x}\right)\right\|^{2}
\end{aligned}
$$


for $\beta>0$, which implies

$$
\left\|\sigma^{-\frac{\nu}{2}}\left(\phi, \psi, \phi_{x}, \psi_{x}, \bar{\phi}, \bar{\psi}, \bar{\phi}_{x}, \bar{\psi}_{x}\right)\right\|^{2} \leq C(1+\delta t)^{-\frac{\lambda-\nu}{4}}\left\|\sigma^{-\frac{\lambda}{2}}\left(\phi_{0}, \psi_{0}, \phi_{0 x}, \psi_{0 x}, \bar{\phi}_{0}, \bar{\psi}_{0}, \bar{\phi}_{0 x}, \bar{\psi}_{0 x}\right)\right\|^{2}
$$

\section{Acknowledgments}

The research of the paper is supported by the National Natural Science Foundation of China (Nos. 11931010, 11871047, 11671384), by the key research project of Academy for Multidisciplinary Studies, Capital Normal University, and by the Capacity Building for Sci-Tech Innovation-Fundamental Scientific Research Funds (No. 007/20530290068).

\section{References}

[1] J. Carr, Applications of Center Manifold Theory, Springer-Verlag, 1981.

[2] Y.Z. Chen, H. Hong, X,D. Shi, Convergence rate of stationary solutions to outflow problem for full NavierStokes equations. Appl. Anal. 98 (2019), no. 7, 1267-1288.

[3] Y.P. Choi, Global classical solutions and large-time behavior of the two-phase fluid model. SIAM J. Math. Anal. 48 (2016), no. 5. 3090-3122.

[4] R.J. Duan, X.F. Yang, Stability of rarefaction wave and boundary layer for outflow problem on the two-fluid Navier- Stokes-Poisson equations, Commun. Pure Appl. Anal. 12 (2013) 985-1014.

[5] S. Evje, T. Flatten, H.A. Friis, Global weak solutions for a viscous liquid-gas model with transition to single-phase gas flow and vacuum, Nonlinear Anal. 70 (2009), no. 11, 3864-3886.

[6] S. Evje, K.H. Karlsen, Global existence of weak solutions for a viscous two-phase model. J. Differential Equations 245 (2008), no. 9, 2660-2703.

[7] H.A. Friis, S. Evje, Asymptotic behavior of a compressible two-phase model with well-formation interaction, J. Differential Equations 254 (2013), no. 9, 3957-3993.

[8] C.C. Hao and H.L. Li, Well-posedness for a multidimensional viscous liquid-gas two-phase flow model, SIAM J. Math. Anal. 44 (2012), 1304-1332.

[9] H. Hong, X.D. Shi, T. Wang, Stability of stationary solutions to the inflow problem for the two-fluid nonisentropic Navier-Stokes-Poisson system. J. Differential Equations 265 (2018), no. 4, 1129-1155.

[10] H. Hong, T. Wang, Stability of stationary solutions to the inflow problem for full compressible Navier-Stokes equations with a large initial perturbation. SIAM J. Math. Anal. 49 (2017), no. 3, 2138-2166.

[11] F.M. Huang, A. Matsumura, X.D. Shi, Viscous shock wave and boundary layer solution to an inflow problem for compressible viscous gas. Comm. Math. Phys. 239 (2003), no. 1-2, 261-285.

[12] F.M. Huang, X.H. Qin, Stability of boundary layer and rarefaction wave to an outflow problem for compressible Navier-Stokes equations under large perturbation. J. Differential Equations 246 (2009), no. 10, 4077-4096. 
[13] M. Ishii and T. Hibiki, Thermo-fluid Dynamics of Two-Phase Flow. New York: Springer-Verlag, 2006.

[14] J.Jung, Global-in-time dynamics of the two-phase fluid model in a bounded domain. arXiv:2012.14612.

[15] Y. Kagei, S. Kawashima, Stability of planar stationary solutions to the compressible Navier-Stokes equation on the half space. Comm. Math. Phys. 266 (2006), no. 2, 401-430.

[16] S. Kawashima, K. Kurata, Hardy type inequality and application to the stability of degenerate stationary waves. J. Funct. Anal. 257 (2009), no. 1, 1-19.

[17] S. Kawashima, A. Matsumura, Asymptotic stability of traveling wave solutions of systems for one-dimensional gas motion, Comm. Math. Phys. 101 (1985), no. 1, 97-127.

[18] S. Kawashima, T. Nakamura, S. Nishibata, P.C. Zhu, Stationary waves to viscous heat-conductive gases in half-space: existence, stability and convergence rate. Math. Models Methods Appl. Sci. 20 (2010), no. 12, 2201-2235.

[19] S. Kawashima, S. Nishibata and P. Zhu, Asymptotic stability of the stationary solution to the compressible Navier-Stokes equations in the half space, Comm. Math. Phys. 240 (2003), 483-500.

[20] H.L. Li, T. Wang, Y. Wang, Wave phenomena to the three-dimensional fluid-particle model, preprint, 2020.

[21] A. Matsumura, K. Nishihara, Large-time behaviors of solutions to an inflow problem in the half space for a one-dimensional system of compressible viscous gas. Comm. Math. Phys. 222 (2001), no. 3, 449-474.

[22] A. Mellet, A. Vasseur, Asymptotic analysis for a Vlasov-Fokker-Planck/compressible Navier- Stokes system of equations, Comm. Math. Phys. 281 (2008), no. 3, 573-596.

[23] T. Nakamura, S. Nishibata, Convergence rate toward planar stationary waves for compressible viscous fluid in multidimensional half space. SIAM J. Math. Anal. 41 (2009), no. 5, 1757-1791.

[24] M. Nishikawa, Convergence rate to the traveling wave for viscous conservation laws, Funkcial. Ekvac. 41 (1998), no. 1, 107-132.

[25] T. Nakamura, S. Nishibata, Stationary wave associated with an inflow problem in the half line for viscous heat-conductive gas. J. Hyperbolic Differ. Equ. 8 (2011), no. 4, 651-670.

[26] T. Nakamura, S. Nishibata, Existence and asymptotic stability of stationary waves for symmetric hyperbolicparabolic systems in half-line. Math. Models Methods Appl. Sci. 27 (2017), no. 11, 2071-2110.

[27] T. Nakamura, S. Nishibata, N. Usami, Convergence rate of solutions towards the stationary solutions to symmetric hyperbolic-parabolic systems in half space. Kinet. Relat. Models 11 (2018), no. 4, 757-793.

[28] T. Nakamura, S. Nishibata and T. Yuge, Convergence rate of solutions toward stationary solutions to the compressible Navier-Stokes equation in a half line, J. Differential Equations 241 (2007), 94-111.

[29] A. Vasseur, H.Y. Wen, C. Yu, Global weak solution to the viscous two-fluid model with finite energy, J. Math. Pures Appl. (9) 125 (2019), 247-282.

[30] X.H. Qin, Y. Wang, Stability of wave patterns to the inflow problem of full compressible Navier-Stokes equations. SIAM J. Math. Anal. 41 (2009), no. 5, 2057-2087. 
[31] G.C. Wu, Y.H. Zhang, L. Zhou, Optimal large-time behavior of the two-phase fluid model in the whole space. SIAM J. Math. Anal. 52 (2020), no.6, 5748-5774.

[32] F.Y. Xu, J. Yuan, On the well-posedness for a multi-dimensional compressible viscous liquid-gas two-phase flow model in critical spaces. Z. Angew. Math. Phys. 66 (2015), no. 5, 2395-2417.

[33] H.Y. Yin, J.S. Zhang, C.J. Zhu, Stability of the superposition of boundary layer and rarefaction wave for outflow problem on the two-fluid Navier-Stokes-Poisson system, Nonlinear Anal. Real World Appl. 31 (2016) 492-512.

[34] L. Yao, T. Zhang, C.J. Zhu, Existence of asymptotic behavior of global weak solutions to a 2D viscous liquid-gas two-phase flow model. SIAM J. Math. Anal. 42 (2010), no. 4, 1874-1897.

[35] L. Yao, C.J. Zhu, Free boundary value problem for a viscous two-phase model with mass-dependent viscosity. J. Differential Equations 247 (2009), no. 10, 2705-2739.

[36] L. Yao, C.J. Zhu, Existence and uniqueness of global weak solution to a two-phase flow model with vacuum. Math. Ann. 349 (2011), no. 4, 903-928.

[37] H.Y. Yin, Converge rates towards stationary solutions for the outflow problem of planar magnetohydrodynamics on a half line. Proc. Roy. Soc. Edinburgh Sect. A 149 (2019), no. 5, 1291-1322.

[38] H.Y. Yin, C.J. Zhu, Convergence rate of solutions toward stationary solutions to a viscous liquid-gas twophase flow model in a half line, Commun. Pure Appl. Anal. 14 (2015), no. 5, 2021-2042.

[39] Y.H. Zhang, C.J. Zhu, Global existence and optimal convergence rates for the strong solutions in $H^{2}$ to the 3D viscous liquid-gas two-phase flow model, J. Differential Equations 258 (2015) 2315-2338.

[40] F. Zhou, Y.P. Li, Convergence rate of solutions toward stationary solutions to the bipolar Navier-StokesPoisson equations in a half line, Bound. Value Probl. 2013 (2013) 1. 